\title{
Translating Advanced Myocontrol for Upper Limb Prostheses from the Laboratory to Clinics
}

This dissertation is submitted for the degree of Dr. sc. hum. at Medical University of Göttingen / Georg-August-Univeristy Göttingen

Promotionsprogramm „Humanwissenschaften in der Medizin“

Submitted by:

Ivan Vujaklija, MSc.

from Belgrade

Göttingen, 2016 


\section{Thesis committee}

Main Supervisor: $\quad$ Prof. Dr. Dr. Dario Farina

Department of Bioengineering, Imperial College London Institute for Neurorehabilitation Systems, UMG

Co-Supervisor: $\quad$ Prof Dr. Russell Luke Institute for Numerical and Applied Mathematics, UG

External Supervisor: Dr. Robert Wendlandt Biomechanics Laboratory, UKSH Lübeck

\section{Examination committee}

Prof. Dr. Arndt Shilling

Prof. Dr. David Liebetanz

Prof. Dr. Florentin Wörgötter

Prof. Dr. Xiaoming Fu

Date of the oral defense: 09.12.2016. 


\section{Preface and Declarations}

The results, outcomes and conclusions presented in this thesis are the result of my own work done at the Institute of Neurorehabilitation Systems at the University Medical Center Göttingen. All collaborations with external partners, as well as the extent of their involvement, have been clearly indicated in the text. My work has been partly funded by the Advanced European Research Council Grant DEMOVE (\#267888).

All experiments involving human subjects have been approved by the local ethics boards and all subjects have signed informed consents prior to their participation. The methods were carried out in accordance to the relevant guidelines.

This thesis has been originally and entirely written by me. Certain portions of the presented material have also been published in form of conference or journal articles elsewhere by me as the principal author or as a co-author. These, as well as all the other relevant parts, have been cited herein adhering to the scientific practice. In cases of previously published material all rights for re-use have been obtained and appropriately labeled.

Herby I declare that I have written this thesis independently, on my own, and with no other aids and resources but those quoted.

Göttingen, 01.10.2016.

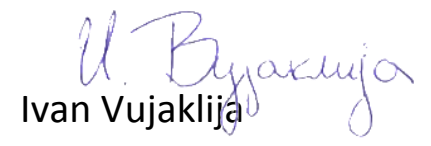


Translating Advanced Myocontrol for Upper Limb Prostheses from the Laboratory to Clinics

If you have an important point to make, don't try to be subtle or clever. Use a pile driver. Hit the point once. Then come back and hit it again.

Then hit it a third time - a tremendous whack.

Winston Churchill 


\section{Table of content}

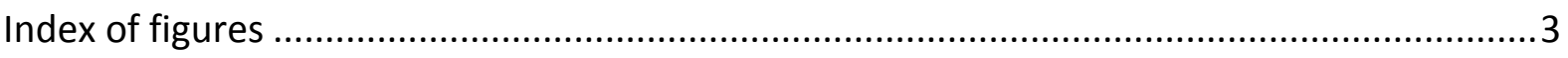

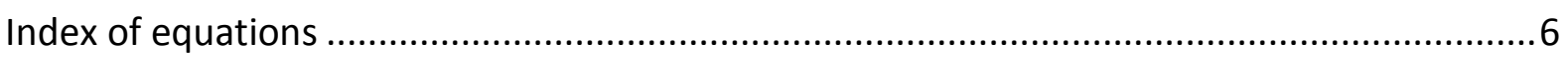

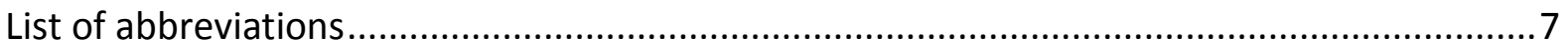

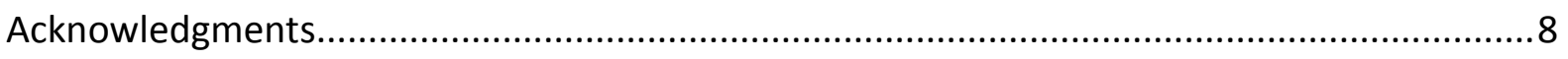

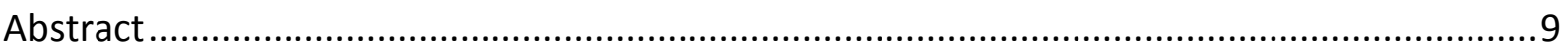

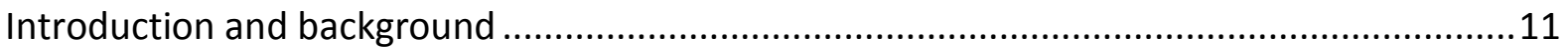

1.1 Shortcomings of the current neurorehabilitation systems.................................. 13

1.1.1 Solutions primarily based on clinical interventions ......................................... 13

1.1.2 Solutions primarily based on engineering techniques ................................... 15

1.2 How to validate new myoelectric systems? .......................................................... 19

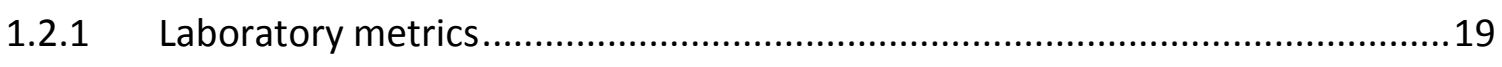

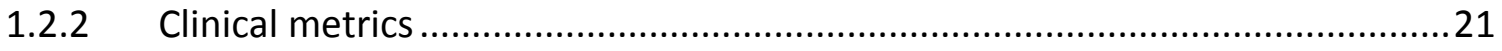

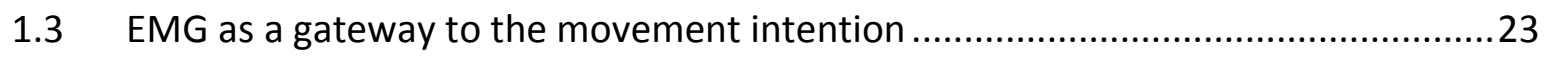

1.3.1 Motor units and generation of EMG...........................................................2

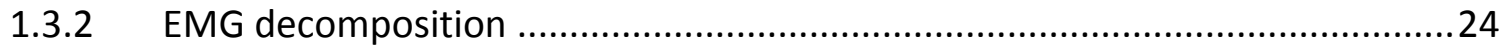

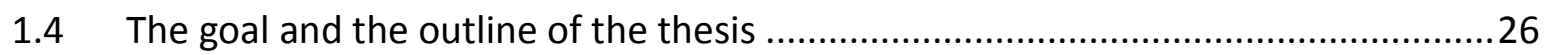

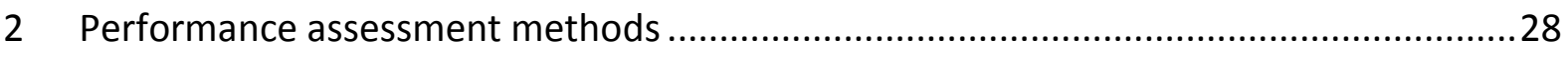

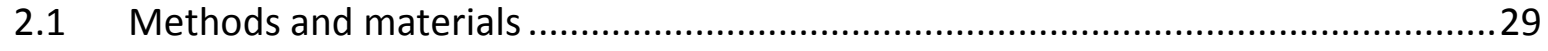

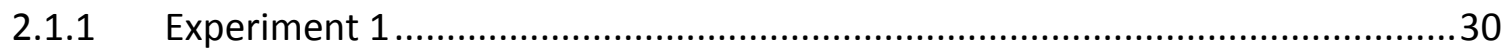

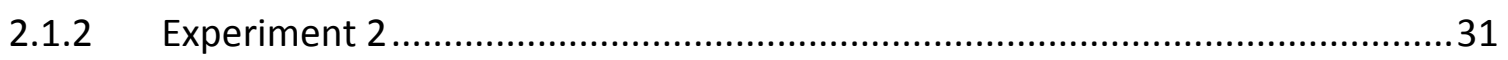

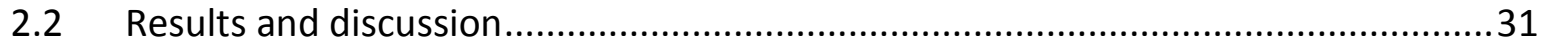

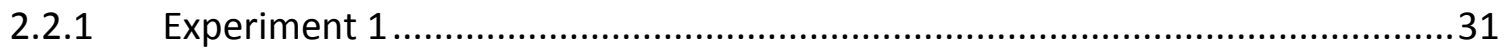

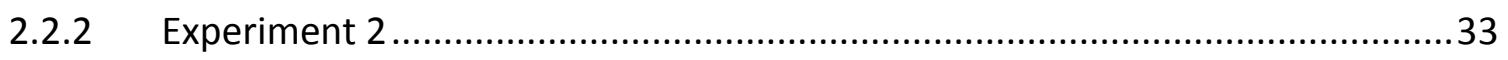

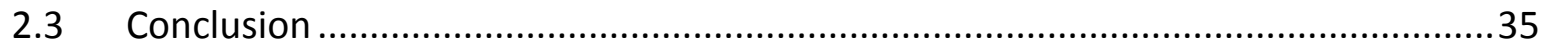

3 Bionic reconstruction enables dexterous prosthetic control after elective amputation

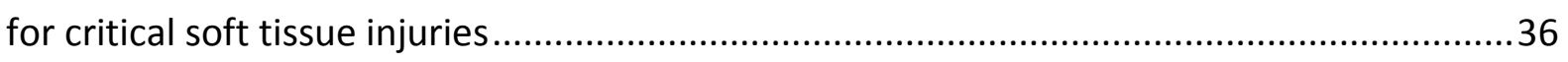

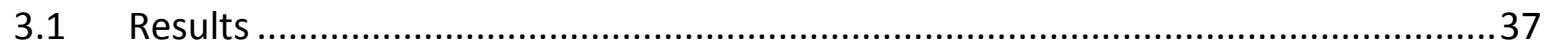

3.1.1 Standardized Functional Outcome Measurements ....................................... 37 
3.1.2 Assessment of Simultaneous, Proportional \& Dexterous Control.....................38

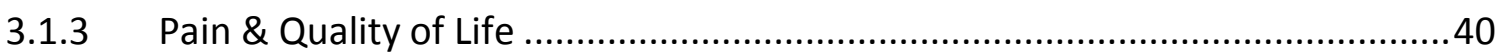

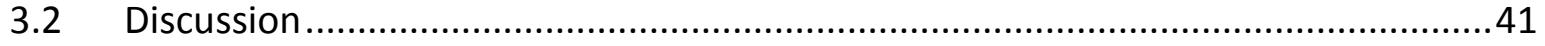

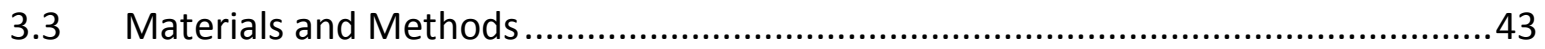

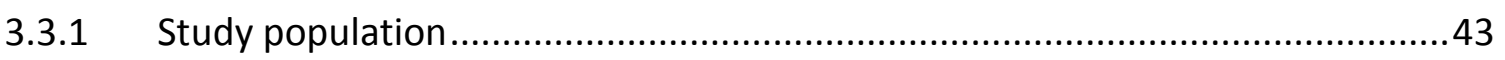

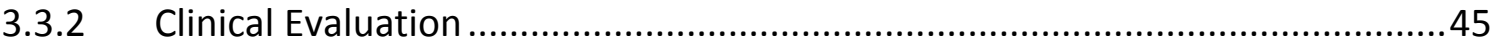

3.3.3 Tech-Neuro-Rehabilitation Program.......................................................... 46

3.3.4 Amputation in Preparation for Hand Prosthesis Fitting .................................47

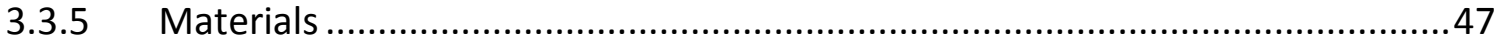

4 Neural decoding for improved force estimation in TMR patients ................................50

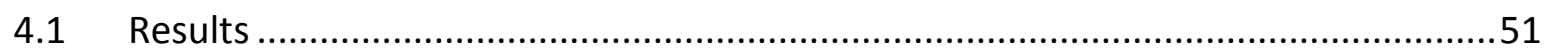

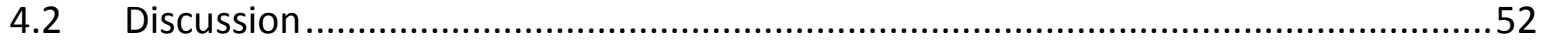

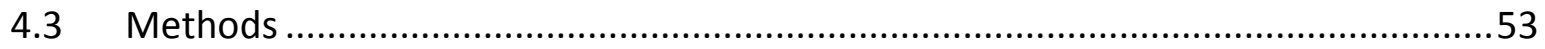

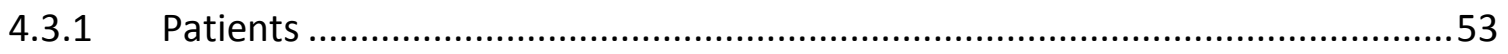

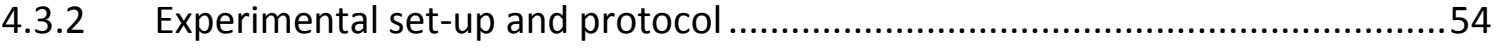

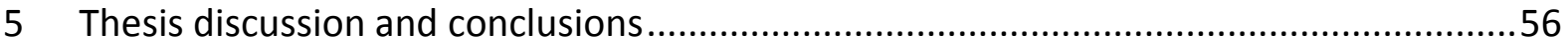

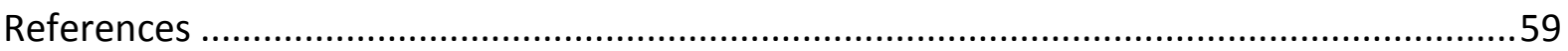

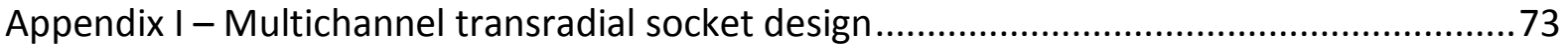

Appendix II - Simultaneous and proportional myocontrol algorithm ..................................78

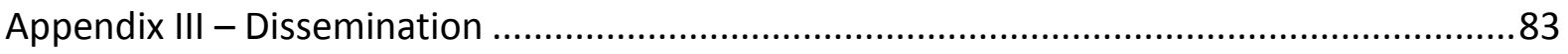

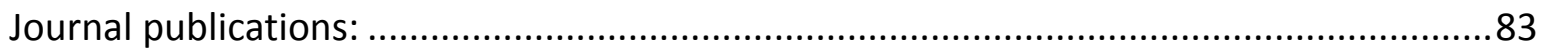

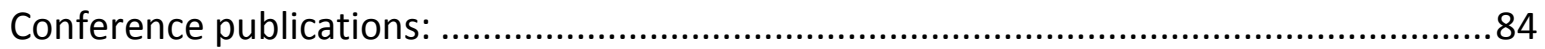

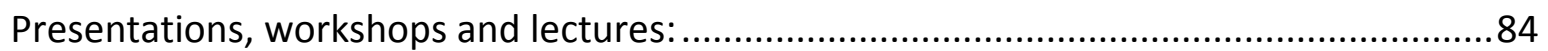

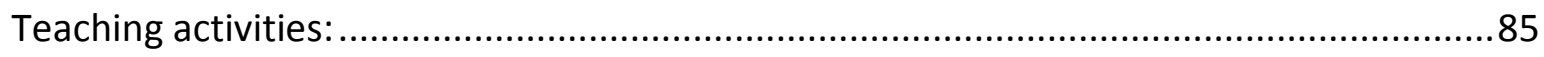

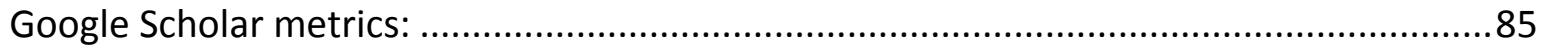




\section{Index of figures}

Figure 1 - Schematic representation of parts belonging to Götz von Berlichingen's hand (16th century), an early iron prosthetic substitution for a transaradial amputee. Even though passive, its fingers can be prepostioned and latched in order to offer certain degree of functionality in combat. (C) $2006 \mid$ de.wikipedia-commons

Figure 2 - Belgrade hand was the first model of a multifunctional externally powered prosthetic limb. It was developed at the institute "Mihajlo Pupin" in Belgrade, 1964. (c)|2006|cyberneticzoo.com-commons

Figure 3 - Advanced prosthetic hand - Michelangelo, Ottobock Healthcare $\mathrm{GmbH}$ - with a rotation unit, extra battery pack and a pair of surface EMG electrodes. This particular prosthesis has an actuated thumb which allows it to close in two ways: pinch grip and key grip.

Figure 4 - TMR operation. Surgeon checks the conductivity using hand held electrical stimulator. Nerves of interest have been marked using yellow strips. (C) $|2006|$ curtecy of CDL

Figure 5 - Assorted clinical evaluation tools (A) Clothes Pin Reallocation Test (B) Box and Blocks Test, and (C) an exemplary jug pouring task from SHAP.....

Figure 6 - Schematic representation of the descending motor pathway starting from the spinal cord (left) and terminating at the muscle (right). Main components of a motor unit are depicted including an exemplary sketch of detectable firings. .23

Figure 7 - Position of passive reflective markers used for motion tracking during the performance evaluation. $10 \mathrm{~mm}$ markers are depicted in green and $20 \mathrm{~mm}$ ones in blue. ....29 Figure 8 - (A) The correlation between the clinical SHAP score and the offline classification accuracy indicates a weak association. The offline scores have been obtained in realistic conditions with the patients wearing their prostheses and training and testing performed on sets of data obtained in different arm positions. Despite the realistic conditions, the associations shown here are not strong. For example, a SHAP score of approximately $\mathbf{4 0}$ may correspond to classification accuracy lower than $70 \%$ or greater than $85 \%$ depending on the user. The SHAP requires precise manipulation over short periods of time which is not captured by this offline metrics. (B) The correlation between the clinical Box and Blocks test and the offline classification accuracy shows almost complete absence of association between the two. For instance, the two patients who achieved the classification accuracies $>95 \%$ were radically different $n$ the number of blocks they could transfer 
Figure 9 - Classification output for two patients with substantially different Box and Blocks outcomes but very similar classification accuracies over all motions. The focus is here on the three hand motions that are most relevant for the Box and Blocks task - hand open, key grip and fine pinch. The offline accuracy for these motions is lower for the subject with the higher clinical score.

Figure 10 - Recorded kinematics with respect to anatomical segments and joints across different sub-groups of SHAP test and CPRT for able-bodied group (1), Patient 1 with classical prosthesis (2) and Patient 1 with advanced prosthesis (3). Notably, in terms of kinematics, Patient 1 was more efficient during the execution of tasks than on average all five able-bodied participants. 34

Figure 11 - Recorded centroid traces of respective anatomical sections across all three axes during the execution of an example task of the SHAP test, the key task, for able bodied group, patient 1 with classical prosthesis and patient 1 with advanced prosthesis 34

Figure 12 - The critical soft tissue injuries suffered by the patients in this study were due to (left) electrocution, (centre) degloving injury, and (right) complications secondary to compartment syndrome. In all cases the reconstructive surgical ladder was attempted first, but with poor functional outcome. .37

Figure 13 - Patient 2, (A) before bionic reconstruction, (B) during hybrid hand training, (C) healed residual limb after elective amputation, and (D) final prosthetic fitting with patient's own customized socket design and art.

Figure 14 - Schematic of the patient training to achieve proportional and simultaneous control at the level of the wrist. First the patient's EMG activity is recorded using eight equidistantly placed surface electrodes during a calibration phase. The gross EMG signal is then decomposed into specific patterns that correspond to seven actions of the prosthetic hand, plus a resting condition. These patterns are uploaded to the prosthetic hand for real time control, which allows for both proportional and simultaneous movements of prosthesis in real-world situations. Details of the used algorithm are describe in Appendix II. 39

Figure 15 - Motor neuron behaviour ramp like activation in patient T1. (A) Amplitude maps of the interference EMG during a contraction of increasing and decreasing force (linear). (B) Prompt that was given to the patient as visual feedback to modulate the intensity of the contraction is shown as a black line. The surface EMG amplitude is shown as a red line. (C) Spike trains of motor neurons decoded through EMG decomposition. (D) Smoothed discharge rates of individual motor neurons with respect to the cue. (E) Instantaneous discharge rate estimated over intervals of $200 \mathrm{~ms}$ computed from the cumulative spike train of the decoded motor neurons for three repetitions of the same task (circles; each repetition is represented with a different color), showing the association between the instantaneous rate of motor neurons and intensity of activity. The behavior of motor neurons during the ramp contractions in this representative example fully reflects the 
general observation on all patients tested and all trials. This is evident from the results on each patient shown in Figure 4 (lower panel).

Figure 16 - Force estimates without (left column) and with (right column) post processing based on averaging over three consecutive past intervals. The plots in the top panels (representatively for patient T4) show the comparison between force estimates based on the EMG envelope (blue) and motor neuron spike trains (red) with respect to the cue (black). The bottom panels show the standard deviations $(\sigma)$ of estimation (colour coded for each of the three patients T4-T6) after linear de-trending when varying the processing interval, without (left) and with (right) post processing.

Figure 17 - Interfacing spinal motor neurons in humans. Following TMR, nerves are redirected to innervate neighbouring muscles which are treaded as biological amplifiers of nerve activity. The spike trains discharged by the innervating motor neurons are decoded by deconvolution of the surface EMG signals. The spike trains are then used to generate the proportional control signal for potential neurorehabilitation applications. The spike trains shown here are extracted from data acquired from patient T1. These spike trains are represented with the sole purpose of describing the general concept proposed in this work. .54

Figure 18 - An example of a negative cast with the second layer made out of cellecast. This particular patient has a rather long and strong stump which enables easy and neat sampling.

Figure 19 - Positive cast after molding ready for refinement and polishing. Strong anchoring line across the front of the elbow can be observed.

Figure 20 - Warmed up thermoplast has to be carefully applied on the positive in order to ensure tight and comfortable fit. This is being done using a vacuum pump during gentle and slow stretching of the material. .75

Figure 21 - Positive cast with a thermoplastic liner still attached and polished polyurethane extension pre-shaped to provide a solid base for casting the outer semi-permanent socket76 Figure 22 - Examples of different semi-permanent sockets built using the presented technique. Four sockets featuring the black Velcro strips are so called open designs intended for users with transcarpal amputations or generally longer stumps .77

Figure 23 - Hybrid myoelectric control algorithm capable of estimating simultaneous and proportional movements. Based on intrinsic dimensionality of the movement, new incoming data point will be forwarded to either sequential estimator (SEQ-E) in case of the single DoF activation or to the simultaneous estimator (SIM-E) if the processed EMG is a product of a multiple DoF activation. C C 2015| IEEE TNSRE 


\section{Index of equations}

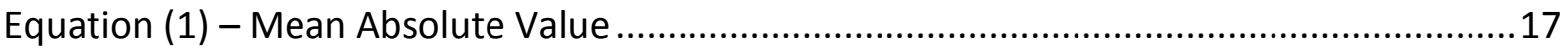

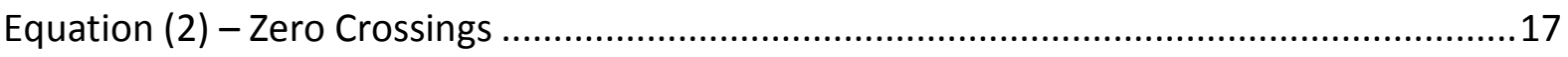

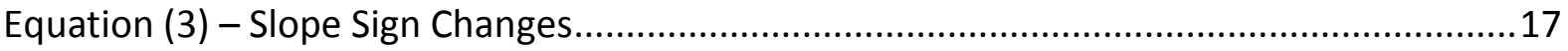

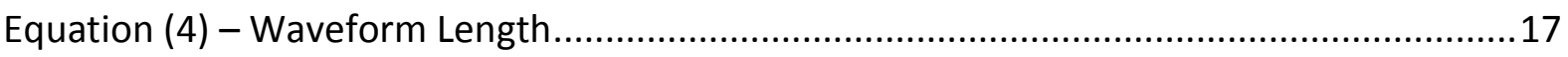

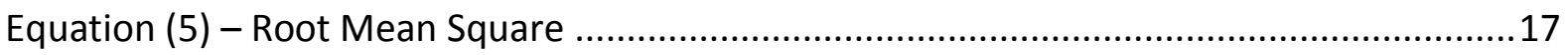

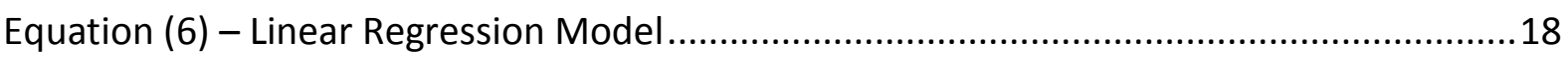

Equation (7) - Linear regression model multiplied by the right pseudo inverse of the measurement matrix, from the right......................................................................... 18

Equation (8) - Definition of the mapping for the pseudo inverse measurement matrix considering a given set and number of channels ........................................................... 18

Equation (9) - Further mapping constrains considering the given $\mathrm{Y}$.................................18

Equation (10) - The set from which linear regression is formed ....................................... 18

Equation (11) - Physiological model of surface EMG as a time-varying convolution of MUAP

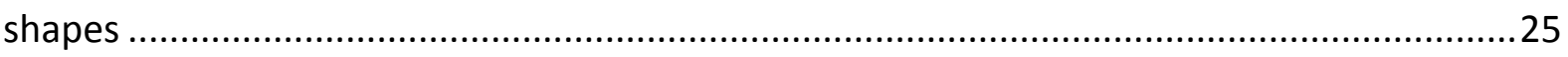

Equation (12) - i-th output of linear, time-invatriant, convolutive, multiple-input-multiple-

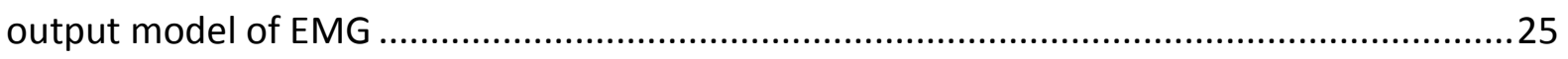

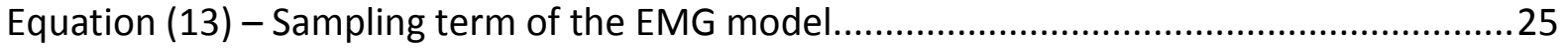

Equation (14) - EMG mixing process presented in the matrix form ...................................25

Equation (15) - Mahalanobis distance measure ..............................................................79

Equation (16) - Linear weighting of the input signals from different channels .....................80

Equation (17) - Variance of $m$-dimensional vector of outputs ............................................8

Equation (18) - Maximization of the generalized Rayleigh quotient in order to obtain

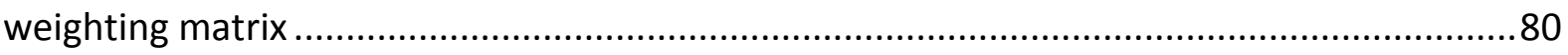

Equation (19) - Constrained optimization problem of equation (18) .................................80

Equation (20) - Quadratic programming transformed into Lagrangian formulation .............80

Equation (21) - Equation (20) differentiated with respect to $W$ and equalized to $0 \ldots \ldots \ldots \ldots . . . .80$

Equation (22) - Equation (21) writtent in a different form .......................................... 80 


\section{List of abbreviations}

\begin{tabular}{|c|c|}
\hline AP & Action Potential \\
\hline ARAT & Action Research Arm Test \\
\hline ACMC & Assessment of Capacity for Myoelectric Control \\
\hline BSS & Blind Source Separation \\
\hline BBT & Box and Blocks Test \\
\hline CPRT & Clothespin Relocation Test \\
\hline CSP & Common Spatial Patterns \\
\hline DoF & Degree of Freedom \\
\hline DASH & Disability of the Shoulder, Arm, and Hand \\
\hline EMG & Electromyography \\
\hline HD & High Density \\
\hline JTHF & Jebsen-Taylor Test of Hand Function \\
\hline MAV & Mean Absolute Value \\
\hline MU & Motor Unit \\
\hline NMF & Non-negative Matrix Factorisation \\
\hline $\mathrm{PE}$ & Proportional Estimator \\
\hline RMS & Root Mean Square \\
\hline SSC & Slope Sign Change \\
\hline SHAP & Southampton Hand Assessment Protocol \\
\hline SoA & State-Of-The-Art \\
\hline TMR & Targeted Muscle Reinnervation \\
\hline VR & Virtual Reality \\
\hline VAS & Visual Analogue Scale \\
\hline WL & Wave Length \\
\hline ZC & Zero Crossing \\
\hline
\end{tabular}




\section{Acknowledgments}

Majority of the work presented in this thesis has been done as a part of the Advanced European Research Council Grant DEMOVE (\#267888) and within the Institute of Neurorehabilitation System at the University Medical Centre Göttingen. Significant portions of the work and the knowledge needed for completing this thesis have been acquired during the research fellowships conducted at Human Oriented Robotics and Control (HORC) Laboratory at Arizona State University (ASU) and Christian Doppler Laboratory (CDL) for Extremity Reconstruction and Rehabilitation at Medical University of Vienna.

I would like to thank Professor Dario Farina for his guidance throughout the course of my PhD studies and more importantly for providing me with a constant advice on how to develop as a researcher in a highly dynamic field. I would also like to extend my gratitude to Professor Russel Luke and Dr. Robert Wendlandt for supporting me in my research and for constructive supervision during the delivery of this thesis.

Crucial clinical experience and overview of the real world impact of my work was made possible due to comprehensive mentoring by Professor Oskar Aszmann to whom I would like to additionally thank for constantly prompting me to re-evaluate ethical principles and focus points of both my work and my private life.

Needless to say, this whole thesis would not be possible without the extensive support during and outside office hours of current and former members of my Institute and hosting organizations. I would specifically like to express my appreciation for encouragements and even more so for discouragements which came before, during and at the very end of my doctoral studies from Marghe, Leo, Chris, and Fra, along with their better halves.

Finally, I would like to acknowledge my family for their endless support and understanding which kept this whole thing on the solid, unbendable pillars high above all the ups and downs that came at it. 


\section{Abstract}

Versatility and dexterity in combination with supreme control makes human hands an unmatched tool for interacting with the world around us. Because of our dependence on hands, we are highly challenged in all aspects of our lives when their functionality is compromised. Considering the high incidence rate of partial or total hand function loss, development of suitable solutions for their substitution are of high priority.

The devastating impact of a missing or a dysfunctional upper limb and the need for solutions to these impairments has been recognized centuries ago. Besides the obvious difficulties which it creates in performing everyday activities, it also reflects on the psychological and emotional state, with difficulties in social re-integration. This can lead to severe long-term consequences in everyday life.

So far, commercial prosthetic limbs have failed to provide a solution capable of delivering intuitive and naturally looking control across several driveable joints. Regardless of being body powered or myoelectrically controlled, these systems depend on rather crude driving mechanisms, limiting the effectiveness of the provided solutions. These limitations eventually lead to rejection and abandonment of the technology. Academic research has addressed this challenge in various ways throughout the last 50 years, though a very limited number of solutions have reached the market. This fact indicates the size and the complexity of the problem of translating the laboratory based systems into the real world environment.

The work presented in this thesis aims at addressing the aforementioned issues by enriching the amount of information that can be used for delivering control inputs over different prosthetic solutions. Theoretically, if the entire neural code sent from the brain to the muscles through the spinal cord could be decoded, its interpretation would allow natural and robust control over virtually any kind of prosthetic system. However, this requires the establishment of an interface that can access this detailed information. Here, several successful attempts to improve the control performance of prosthesis by advanced information methods of identification of the properties of the neural drive to muscles have been described and applied to already established prosthetic solutions. Focus has been put on translational potentials of these approaches and challenges which arise when systems initially developed in laboratory environment are further put to test in clinical setting.

First, an in depth re-evaluation of the way in which the functional prosthetic assessment has been performed in academic and clinical studies is presented through a set of experiments. Comparison between the most commonly used offline evaluation technique and several typically applied clinical tests has been performed on a pool of transradial amputees. A poor correlation was found between the two sets of performance metrics, indicating the need of using more meaningful assessment scores in academic research to evaluate novel myoelectric systems. In addition, a kinematic analysis has been made during the execution 
of selected clinical tests, indicating that even the well-established clinical tools fail to completely evaluate all the aspects of the tested systems.

The second set of experiments focused on advanced myoelectric control for patients who have sustained critical soft tissue injuries. So far, there has not been a suitable solution for recovering function in these severe cases. Here, through a combination of surgical interventions and rehabilitation technics, an interface for accessing myoelectric information sufficient for advanced control of sophisticated prosthetic technology has been established. A case series is presented to prove that surgical and engineering solutions can be combined for solving open clinical challenges through means of bionic reconstruction.

The final set of experiments was designed to test the possibility of providing precise proportional control from motor unit spike trains originating in the spinal cord. The motor unit discharge patterns were decoded from high density surface EMG recordings obtained from reinnervated auxiliary muscles in the proximity of a high level amputation. This approach provides an enhanced prosthetic function across a difficult pool of transhumeral patients.

Results presented here emphasise the importance of clinical testing of myoelectrical systems and provide an insight into the complexity of the translational challenges which arise once laboratory systems are exposed to the reality of clinical environment. The data provided in this thesis support the idea that advanced control approaches can be translated to effective clinical solutions even in cases that were earlier considered beyond the reach of myoelectric technologies. Finally, a new generation of neural interfaces, relying on the decoded neural drive to muscles, has been shown to be able to deliver highly refined control, and thus potentially revolutionize the way the prosthetic devices are driven. 


\section{Introduction and background}

Considering that virtually all of our daily activities require a certain level of hand manipulation, it is not surprising that upper limb injuries are among the most common ones. Indeed, a significant portion of overall injuries treated in the emergency rooms around the world involve upper extremities [1]-[3]. Most often they occur during work [4], [5], at home [2] or while performing sports [6].

The consequences of these incidents may lead to prolonged disabilities which can further affect the mental and social state of the patients. The effects of these complications can result in difficult reintegration into the society.

The severe difficulties of a life without a limb have been recognized centuries ago [7]. The early ideas of artificial substitution appeared appealing, even in their very simple (Figure 1) or purely cosmetic form. Evolution towards more functional solutions was just a matter of time and the high demand yielded the first body-powered systems. These simple, yet effective, devices delivered much needed support in everyday life of an amputee through a set of cable driven joints. Their effectiveness has proven so significant that some of their fundamental principles can still be found in modern prosthetics. Roughly $50 \%$ of the current market share of the upper limb prosthesis is based on cable driven systems [8].

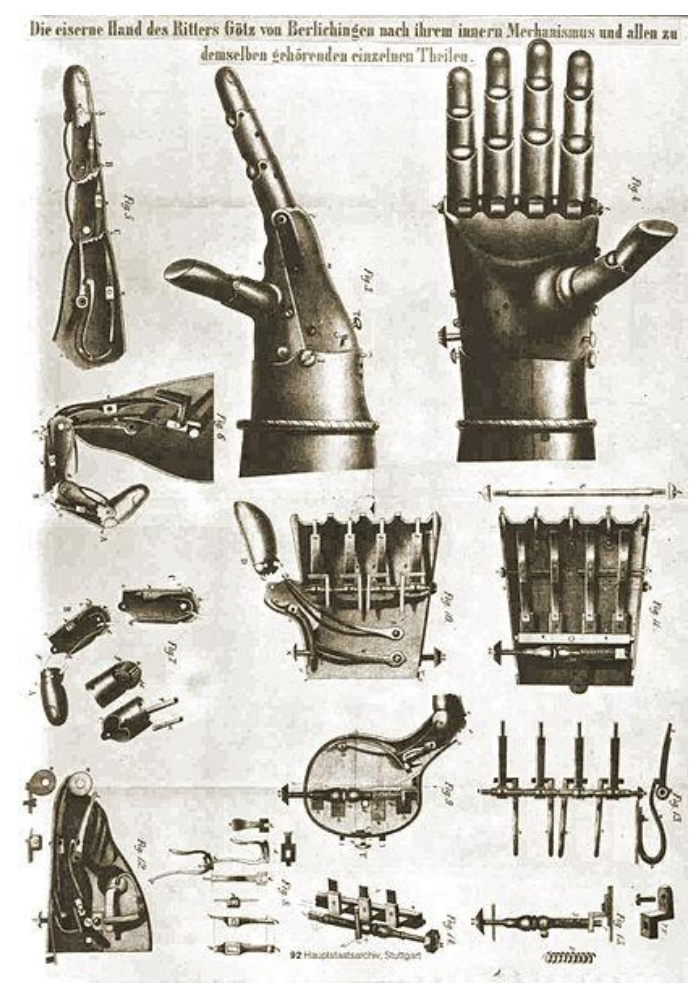

Figure 1 - Schematic representation of parts belonging to Götz von Berlichingen's hand (16th century), an early iron prosthetic substitution for a transaradial amputee. Even though passive, its fingers can be prepostioned and latched in order to offer certain degree of functionality in combat. 이2006|de.wikipedia-commons 
The turn of the last century brought the first pneumatic hands which were soon accompanied by the electrically powered ones. Some forty years later, at the end of the Second World War, basic myoelectric prostheses were developed, revolutionizing the way the neurorehabilitation technologies are interfaced [9]. Researchers soon embraced this concept which allowed linear translation of the electrical activity of the residual muscles of the stump into the velocity of closing and opening of mechatronic gripers. By late 50s, the first myoelectric devices became available on the market and the concept of direct proportional control is still present in current commercially available systems, primarily due to its simplicity and robustness.

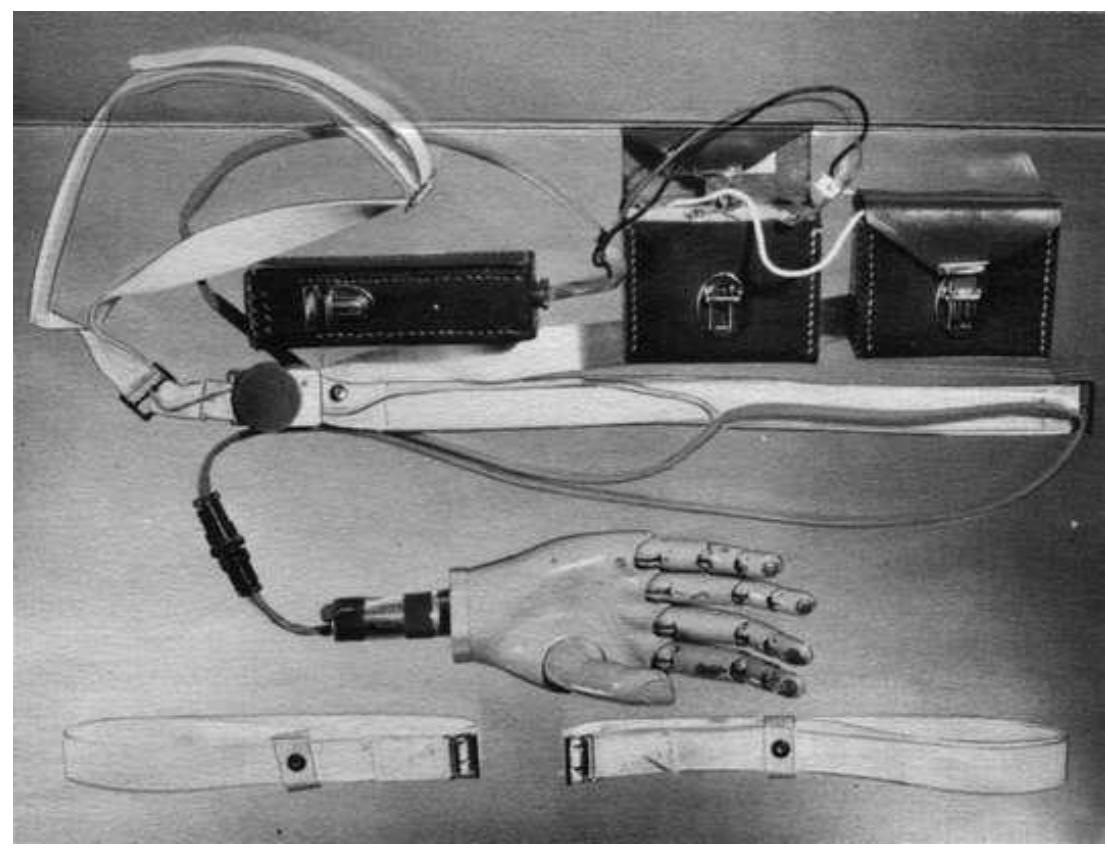

Figure 2 - Belgrade hand was the first model of a multifunctional externally powered prosthetic limb. It was developed at the institute "Mihajlo Pupin" in Belgrade, 1964. 이2006|cyberneticzoo.com-commons

With advancements in technology, pneumatic prosthetic devices became capable of driving several joints and grip types (Figure 2) offering functional options, even for users with more distal deficiencies. However, the supporting control paradigm was crude, lacked robustness and required specific anatomical features, dexterity and cognitive effort from the patient [10]. Myoelectric interfaces allowed a state-based control to be introduced. The user is fitted with a multi articulated prosthesis using two control sites which are responsible for driving a single degree of freedom (DoF). When another DoF is required, such as a different joint control or a grip type, a switch in the form of a co-contraction of the muscles beneath the two recording sites is introduced and the control state of the prosthesis is changed. In this way, the user is able to robustly cycle through as many DoFs as they are available. Even though cumbersome, unintuitive and cognitively demanding, this paradigm is still dominant on the market. 
Light weight dexterous prosthetic hands and wrists (Figure 3) are nowadays commercially available due to recent advancements in micromachining and material design. Alongside tiny, precise and low powered electromotors, these technological flagships delivered some of the state of the art solutions in modern robotics. Research and implementation of more intuitive and dexterous myoelectric control algorithms intensified once high-speed processing units with the top end battery management and large memories in small housing were made broadly available (Figure 3 ).

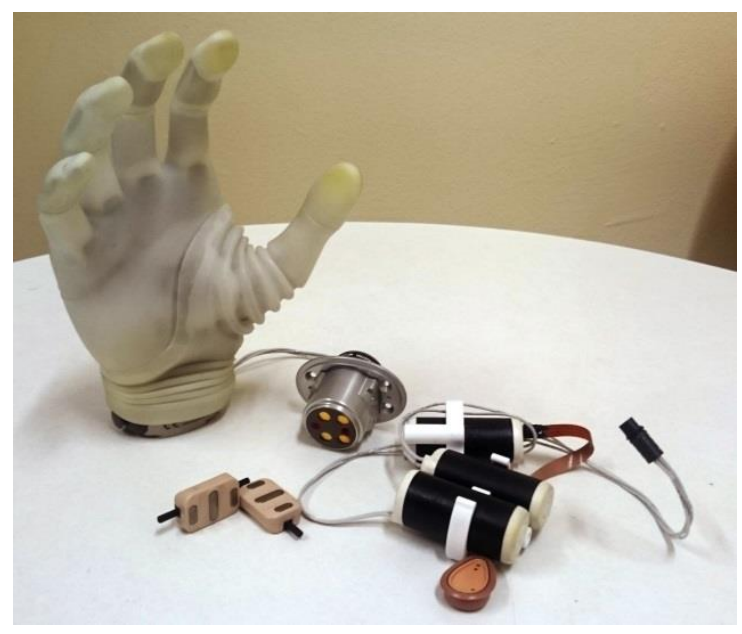

Figure 3 - Advanced prosthetic hand - Michelangelo, Ottobock Healthcare GmbH - with a rotation unit, extra battery pack and a pair of surface EMG electrodes. This particular prosthesis has an actuated thumb which allows it to close in two ways: pinch grip and key grip.

\subsection{Shortcomings of the current neurorehabilitation systems}

Various attempts were made to provide improved and functional neurorehabilitation solutions to individuals with upper limb deficiencies. However, this has proven to be quite a challenging task. One of the main difficulties lies in the fact that the disabilities of such a highly articulated and complex system such as human arm, tend to come in a variety of forms, shapes and limitations. Therefore, a simple "one fits all" solution doesn't seem to be an option here at the moment. Considering strictly the cases in which neural drive stays intact, still quite often the current neurorehabilitation technologies tend to underperform or even not to be able to provide any functional benefit at all.

\subsubsection{Solutions primarily based on clinical interventions}

Namely, the most obvious reason for poor performance comes from a general lack of sources from which necessary neural information can be retrieved. This creates significant difficulties in providing functional solutions to high level amputees. Recently, targeted muscle reinnervation (TMR) technique (Figure 4) has been introduced with the idea of rerouting the nerves which originated in the missing limb to the muscles in the proximity of the amputation [11]. 


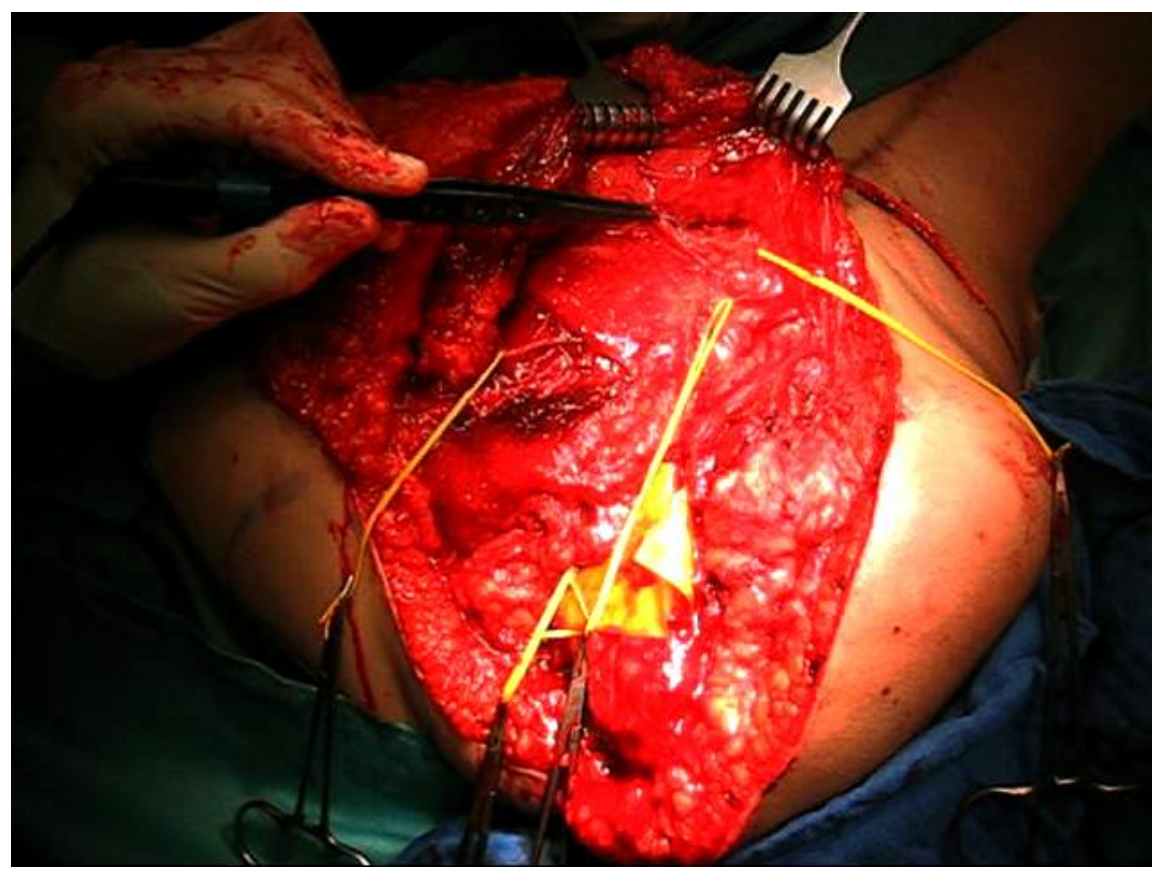

Figure 4 - TMR operation. Surgeon checks the conductivity using hand held electrical stimulator. Nerves of interest have been marked using yellow strips. (C) $2006 \mid$ curtecy of CDL

The main motivation for this procedure is that muscles can be seen as amplifiers of nerve activity [12]. Reinnervated muscles will not be used for their functional role, but rather as sources of EMG signals [13]. During the surgical procedure the nerves of interest are located and end neuromas are excised. The residual nerves are trimmed back until healthy fascicles are observed [14]. The remaining nerves are then appropriately transposed and fixed using locally available anatomy. Native innervation of the target muscles is disrupted and confirmed using local stimulation (Figure 4). The mobilized segments of the remaining nerves intended for transfer are then coapted to the motor nerve entry point using 6-0 or 7-0 polypropylene suture under appropriate magnification [14]. Four to six weeks post-op patients can expect to be able to wear again their original prosthesis, while after three to six months the full reinnervation should take place [15]. The obtained activation does very well reflect the neural activity of the missing limb and that has been shown in various practical applications [16]-[18].

Even though this solution restores the possibility for non-invasive access to the original neural drive, still a large number of DoFs, which need to be addressed, deems the classically provided "one source - one DoF direction" control to be crude. Additionally, since only four main nerves serve the entire arm: Median Nerve, Ulnar Nerve, Radial Nerve, Musculocutaneous Nerve, the maximum number of control sights limits this control algorithm to drive up to six joint directions using peripheral branches and some rehabilitation tricks [19]. An example of nerve transfer matrix is shown in Table I. Even though an improvement, this is still not fully satisfactory for the majority of users especially given the rough end control which is being delivered. 
Translating Advanced Myocontrol for Upper Limb Prostheses from the Laboratory to Clinics

Table I - Nerve transfer matrix example in glenohumeral patient with targeted prosthesis function

\begin{tabular}{|c|c|c|c|}
\hline Source Nerve & Target Nerve & Target Function & Prosthesis Function \\
\hline N. Musculocutaneous & N. Pectoralis Clav. & Elbow Flexion & Elbow Flexion \\
\hline N. Medianus & N. Pectoralis stern. & $\begin{array}{l}\text { Finger Flexion / } \\
\text { Wrist Pronation }\end{array}$ & $\begin{array}{l}\text { Fast: Wrist Pronation } \\
\text { Slow: Wrist Extension }\end{array}$ \\
\hline N. Medianus & N. Pectoralis Abd. & Wrist Flexion & Elbow Extension \\
\hline N. Ulnaris & N. Pectoralis Minor & Fist & Hand Close \\
\hline N. Radialis & N. Thoravodorsalis & Finger Extension & Hand Open \\
\hline
\end{tabular}

Various difficulties in applying neurorehabilitation technologies can also be observed in individuals where poor stump management has been done during initial amputation treatment. In these cases standard surface EMG based solutions are not able to retrieve sufficient amount of neural drive information to run properly. Similarly, there may be pathologies or structural shortcomings present at the muscular level which partially or even completely prevent this type of users to have any functional systems applied. In these cases it is sometimes possible to perform surgical or correctional interventions which will either on its own or in combination with assistive neurotechnologies be able to deliver certain functional benefits. These include such techniques as tendon transfers [20], [21], a variety of flaps [22]-[25], digit and toe transfers [26]-[28], and skin grafting [29], [30].

In some particular yet not so uncommon cases, functional state of the patient might be severely compromised even though anatomically there is a significant portion of the limb which remained. In those situations offering a proper functional solution might be rather difficult. For instance, this is commonly the case in patients who have suffered plexus or critical soft tissue injures.

It should be noted that, as an alternative to the prosthetic devices, the hand transplantation represents a functional substitute with superior visual appeal and on top of it, integrated sensory function [31]. However, it is associated with the lifelong immunosuppressant therapy, lengthy rehabilitation, loss of grip force, and high risk of complications leading to the possible rejection [32], [33]. These issues are further combined with very high costs and necessity of treatment in specialised clinics. Current recommendations for hand transplantation target bilateral transradial amputees pass the stage of family planning, with superior mental health and with explicit evidence of dedication and devotion to rehabilitation.

\subsubsection{Solutions primarily based on engineering techniques}

Even in the cases where there is a sturdy interface to the neural drive, current neurorehabilitation technologies seem not to fully deliver. Surveys conducted in the past 20 years indicate significant rejection rates of all types of upper limb prosthetic solutions [34]-[39]. Depending on the user population at question, rejection rates of 
myoelectric devices vary from $25 \%$ to over $50 \%$. In case of body powered devices $35 \%$ of prosthesis ends up being discarded by their users. Some consolation can be found in the fact that these numbers have been stagnating compared to previous periods [40], [41] and this has been mainly attributed to the introduction of modern technology. Still the highest number of complaints by users is directed towards cumbersome, unreliable and unintuitive control of all prosthetic solutions - old or new.

The current industrial state-of-the-art (SoA), from a technical control point offers quite a rudimental solution. The implemented principle didn't change much from 1960s when sensed amplitude of the EMG signal was linearly translated into the directional velocity of the given DoF [9]. Nowadays market is dominated by two electrode systems, monitoring available antagonist muscle groups. Depending on the simple threshold breach one or the other direction of the controlled DoF is activated. This approach is rather robust and it offers quite a steep learning curve for novice users. Though, this is only a case in systems offering single DoF functionality such as simple grippers. On the other hand, if the dexterity of the prosthesis is extended beyond that, multiple DoF control becomes rather cumbersome. These kinds of systems mostly rely on state machine schemes which are guiding users through each controllable DoF with a switching signal. Switching signals can be myoelectric based, such as co-contraction of monitored sites [42], quick repetitive activation of the dedicated site [43] or prolonged contraction of single muscle group [43]. Conversely, they can also be implemented in a form of a simple button on the prosthesis [44], a digital signal sent from an external device, actual physical prepositioning of a prosthetic joint or an external digital trigger originating from an active or passive device in prosthesis' proximity [45]. Regardless of the switching paradigm, all of these solutions are suffering from the intrinsically cumbersome underlying control principle that is driving the device. Namely, if one was to control the elbow joint after prepositioning the wrist, the system would force the user to cycle through several available grasping types only to gain the control over it.

Researchers and developers have been addressing the issue of cumbersomeness for the last decades by combining and developing different engineering techniques. Majority of the solutions have been relying on the machine learning methods specifically adapted for the problem at hand. The assumption that distinguishable and repeatable EMG signal patterns exist among different motor tasks [46], [47] gives an opportunity for selecting one of many well established estimators to be used in deciding which DoF is being activated. The estimation methods can be roughly divided into classification and regression approaches. The first ones yield discrete outputs used as class labels, while the later fit smooth curves to, for instance, force functions.

Ease of implementation has put classification based algorithms as top contenders for revolutionizing myoelectric control. Namely, the early works from 1960s and 1970s 
[48]-[50] relied on discriminant analysis for differentiating as many as three DoFs from EMG motion data sets described solely by the global muscle activation. In early 1990s, first step was made towards extracting more of the available information from the EMG [46]. EMG signals were split into $300 \mathrm{~ms}$ interlaced windows which were used from calculating the following signal features (adapted from [46]):

$$
\begin{array}{ll}
\begin{array}{l}
\text { Mean Absolute } \\
\text { Value }
\end{array} & M A V:=\frac{1}{N} \sum_{k=1}^{N}\left|x_{k}\right| \\
\text { Zero Crossings } & Z C:=\frac{1}{N} \sum_{k=1}^{N} c, c \\
& = \begin{cases}1, & \text { if } x_{k} \cdot x_{k+1}<0 \\
0, & \text { otherwise or if }\left|x_{k}-x_{k+1}\right|<0.01 V\end{cases} \\
\begin{array}{l}
\text { Slope Sign } \\
\text { Changes }
\end{array} & S S C:=\frac{1}{N} \sum_{k=1}^{N} c, c \\
\text { Waveform Length } & = \begin{cases}1, & \text { otherwise or if }\left|x_{k}-x_{k+1}\right|<0.01 V \\
0, & W L:=\frac{1}{N} \sum_{k=1}^{N}\left|x_{k}-x_{k-1}\right|\end{cases}
\end{array}
$$

with $x_{k}$ being the $k^{\text {th }}$ sample of the observed $N$ samples long time window. $x_{k}$ has been obtained through a process of quantization by analogue-digital converter and as such represents a single real number equivalent to the EMG voltage for a given channel during the $k$-th sampling time frame. These features were extracted from each channel and once used as a set (in myoelectric pattern recognition community referred to as Hudgins time domain set [51]) they contain the information on both amplitude (MAV, WL) and frequency (ZC, SSC, WL) of the sensed EMG. Alternatively, MAV is in some myocontrol systems substituted by root mean square (RMS) value of the signal:

$$
R M S:=\sqrt{\frac{1}{N} \sum_{k=1}^{N}\left(x_{k}\right)^{2}}
$$

The basic concept for this kind of control algorithms set by Hudgins, Parker and Scott [46] remained in essence the same to the day. Most of the work has been directed towards different classifiers [52]-[54] and extending the feature sets [55], [56].

Roughly a decade ago an alternative to classifiers with hard boundaries and discrete outputs was proposed in a form which allows direct estimation of the movements speeds or forces of desired DoFs. This is done by performing a regression between the input signal features and the target outputs. Assuming the linear relation between the two, a simple linear regressor can be implemented. It would linearly map the input 
features $\boldsymbol{x}$ with the desired state of the actuators contained within the target vector $\boldsymbol{y}$ using a weight matrix $W$ :

$$
\boldsymbol{y}=W^{T} \boldsymbol{x}
$$

Considering the three DoF case, $\boldsymbol{y}$ would be a three and $\boldsymbol{x}$ would be a $n$-dimensional vector. Therefore, the matrix of weights will be $W \in \mathbb{R}^{n \times 3}$. Fit with the least squared error between all measurements $X$ and all targets $Y$ can be obtained by multiplying the previous equation with the right pseudo inverse of the measurement matrix, from the right:

$$
X_{r}^{-1} Y=W^{T}
$$

where for a given set and number of channels $N$, following mapping is defined:

$$
X_{r}^{-1}: \mathbb{R}^{3 \times N} \rightrightarrows \mathbb{R}^{n \times N}
$$

such that for given $Y \in \mathbb{R}^{3 \times N}$ :

$$
Y X_{r}^{-1}:=\left\{W^{T} \in \mathbb{R}^{3 \times N} \mid W^{T} X=Y\right\}
$$

In general, $W^{T}$ is represents a part of a whole set of weight matrices $W^{T} \in Y X_{r}^{-1}$ and linear regression that is formed is just a selection from this set:

$$
W_{L R}^{T}:=Y X^{T}\left(X X^{T}\right)^{-1} \in Y X_{r}^{-1}
$$

It should be noted that such selection is reasonable only for linear models with Gaussian noise.

This, of course, represents the most straight forward regression approach, which can be further extended by regularization or application of the kernel trick for generating non-linear estimations [57]. For instance, in what is commonly known in the field as the kernel trick (kernel ridge regression), the kernel extension of Tikhonov regularization method of ill-posed problems is made [58]. In this way the same error function as in linear regression is minimized (6), though not in the in the input space, but rather in the space formed by potentially nonlinear mapping of the inputs [57]. For myocontrol applications, Gaussian kernel function is commonly used.

Both classifiers and linear regression with its derivatives represent the fully supervised methods, meaning that they require meticulously labelled target data corresponding to each input vector. This can be achieved by tracking the kinematics of the sound limb during the bilateral mirrored movements [57], [59]-[61]. Though, understandably, a certain degree of error will be introduced in this way, which will highly reflect on the performance of the system. However, once a more model based approaches are considered, semi-supervised regressors may be applied. For instance, derived from a muscle synergy driven model [62], a non-negative matrix factorization (NMF) algorithm 
[63] can be used to generate the desired mapping by only having the information on which DoF was active at the time and not necessarily the exact force trajectories [64].

Main advantage of regression based approaches is that, compared to classifiers, they are not providing just labels as their outputs, but rather give the estimation of the actual physical values. In addition, these methods can intrinsically extrapolate from single DoF training data and predict the multi DoF movements [65]. This is in particular important for providing more natural control of the prosthesis. Unfortunately, regression based systems are less resistant to noise. Even though through some training, smart unintuitive mapping and high density (HD) EMG recording, the number of controllable DoFs can be as high as seven in able bodied subjects [66], two DoF control seems to be the reasonable framework to work with [67].

Recently, a myocontrol system combining the two approaches has been proposed [67]. Two estimators, one based on a classifier and one on a regressor, are designed and trained in parallel. With every new feature vector, based on its distance from the centre of the feature data point clouds of each class, a simple threshold based decision is made determining whether the intended motion is single DoF (if the point falls within one of the classes) or multi DoF (if it lands between the clouds). Depending on the decision, all single DoF motions are forwarded to the classifier for further processing, while all the others are handled by the regressor. In this way precise movements are estimated by a strict, more robust estimator while gorse, usually prepositioning movements, are dealt by softer control method able of driving combinations of DoFs.

\subsection{How to validate new myoelectric systems?}

Assessing a performance of a neurorehabilitation technology is not an easy task. There are numerous parameters involved, and getting a sound and a fully describing score is a rather complex endeavour. Finally, the most valid evaluation in the end comes in the form of a user's subjective opinion after prolonged exposure to the technology. Understandably this is not always a possible way to go when deciding which method or system should be further pursued, therefore academia and clinics have developed their own ways of quantifying the delivered performance.

\subsubsection{Laboratory metrics}

Researchers working on the developments of myoelectric prostheses have come up with numerous ways of assessing the performance of their newly developed systems. Roughly, these scores can be separated into those which are strictly quantifying the system's behaviour using offline metrics and those based on online assessments in virtually generated scenarios. 
As aforementioned, academia is mostly developing solutions for dexterous control which are as control outputs delivering estimations in form of a discrete class or a physical value such as end effector force or position. With respect to that, the most common offline performance scores used are either classification accuracy [68] or $R^{2}$ error with respect to a given prompt [53] respectively. In the first case, the score is formed based on the amount of correct estimates that the tested classifier is able to make, given the new unseen data. The later directly compares the estimated physical value to the reference cue generating the score in the form of a widely used coefficient of determination $R^{2}$.

With the advancements of computer graphics several very emerging virtual reality based evaluation benches have been proposed. Their purpose is to simulate the real, online use of prosthesis at various levels of abstraction. The main advantage is that they still provide all the benefits of the research environment while not demanding the full system implementation. In addition, time consuming and quite effort heavy socket fitting can be avoided as well as the optimization phase required for transferring the algorithm, or its parts, to an embedded hardware. These VR benches can be rather abstract compared to the intended control [69] involving some sort of a computer avatar which needs to be steered across the screen. Though, they can also be sufficiently simple video games prompting users to drive various cursors in order to reach given targets presented to them [53], [64]. Lastly, VRs can even consist of a digital replica of the actual prosthesis which then needs to be placed and matched to a target posture [70]. Regardless of the setup, all these are aiming at analysing ones performance for a given control algorithm during the activation of a specific DoF or a combination DoFs.

The online systems are superior to the offline evaluations since they directly set the user in the loop and therefore account for human adaptation to the system. Parameters such as completion rate, path efficiency, number of overshoots or throughput, provide a solid quantitative evaluation of online performance. Further, [71] introduced the Fitts' law [72] in evaluating myocontrol. Through some iterations [65], [73], [74], a single statistical measure has been proposed to characterize a myocontroller online. Nonetheless, even if some of these test benches offer realistic testing scenarios, they have limitations. For example, weight bearing by the prosthesis and stump dynamics causing pressure changes within the socket fitting are important realistic factors of influence [75], not included in these tests. On the other hand, VR systems have found relevant applications in patient training [76], [77] and can be combined with table-top prosthetics [15]. 


\subsubsection{Clinical metrics}

Clinicians and rehabilitation specialists are relying on their own set of metrics and questioners for evaluating patient's functional improvement given a certain neurorehabilitation technology. These tests have been mainly based on the techniques used for post stroke evaluation and the influence of the upper limb rehabilitation. They mainly consist of a various abstract object manipulation and tasks resembling those of everyday activities. The main outcome of the majority of these tests is whether or not the subject is capable of actually completing them and in which time. In a smaller number, some of the clinical tests account for subjective and personal perception of how the tasks have been executed and therefore require a presence of a certified examiner.

The most commonly used and perhaps the simplest of the clinical evaluation techniques is box and blocks test (BBT) shown in Figure 5B. It is intended to quantify the severity of the upper limb deficiency in a very straight forward manner. It requires from a subject to transport as many of the wooden blocks as possible in one minute while standing, from one side of the barrier to the other. The final score is formed as an average of three such repetitions. Though simple and effective, this test focuses strictly on a limited number of DoFs and requires minimal amount of skill by the subject.

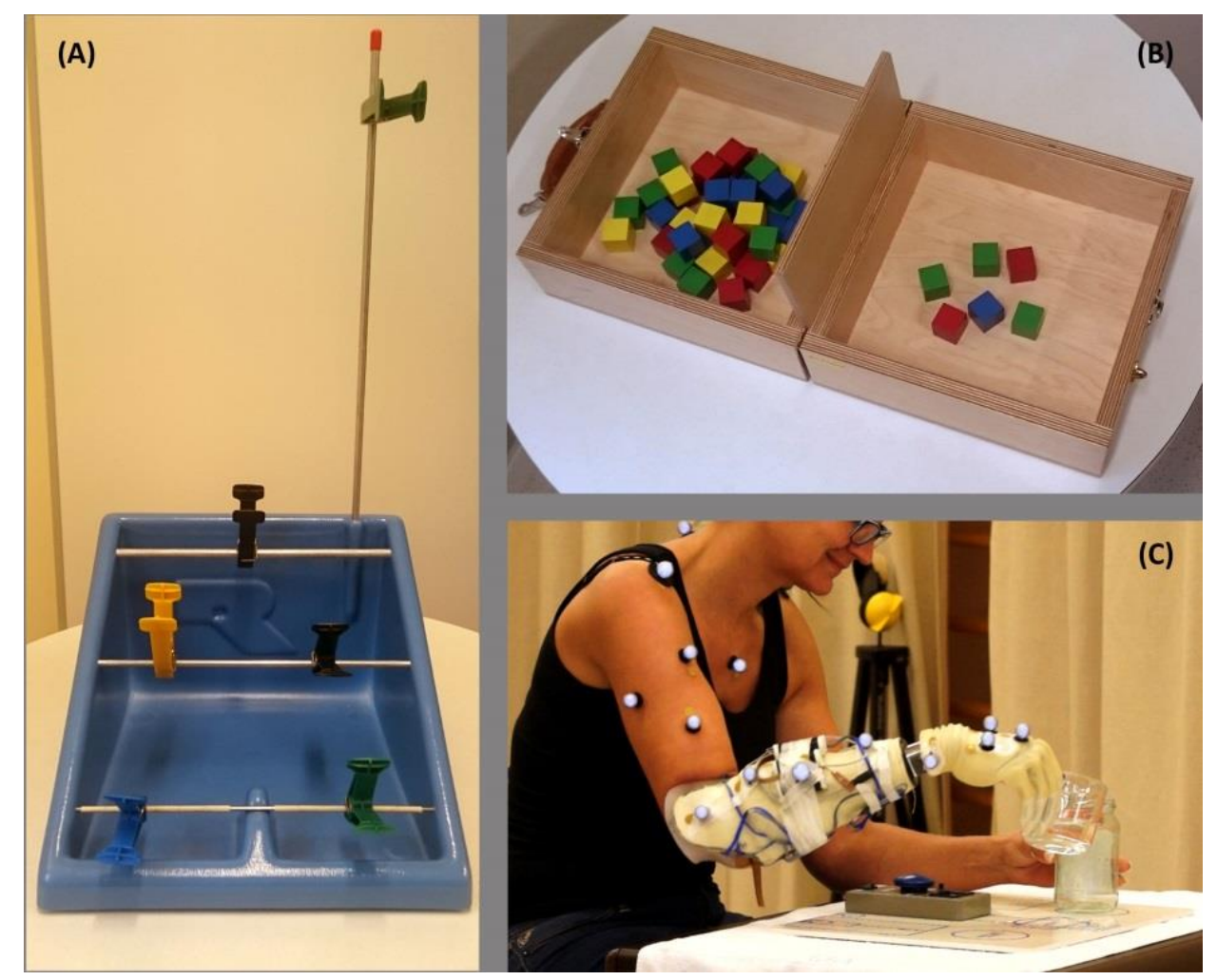

Figure 5 - Assorted clinical evaluation tools (A) Clothes Pin Reallocation Test (B) Box and Blocks Test, and (C) an exemplary jug pouring task from SHAP 
For assessing user's and system's performance in tasks which typically require wrist activation, an adaptation of Royal Graded Pinch Exerciser is used. Namely, clothes pin relocation test (CPRT) depicted in Figure 5A prompts a subject to move a set of clothes pins of various resistances from a horizontal to a vertical bar. Being primarily a rehabilitation device, an exact procedure for performing an assessment has not been defined. Still, majority of clinicians agrees on using four clothespins of different resistances (1, 2, 4 and $8 \mathrm{lbs}$.) and instructs subject to relocate each of them from the lowest horizontal bar to the most suitable position along the vertical bar. The overall time needed is recorded and averaged over three repetitions in order to form the final score. The entire test is performed while standing.

The most elaborate clinical upper limb assessment tool is the Southampton Hand Assessment Protocol (SHAP) [78]. It evaluates user's performance during an execution of 26 different tasks which include six different grip types and their combinations. The final score is number which ranges from 0 to 100 . Reaching the ultimate side of the scale corresponds to a healthy hand function, while the opposite represents an absence of it. The achieved score takes into the account one's ability to complete the task and the time that it took. Being a very elaborate test, SHAP's down point is that it tends to be lengthy and tiring, especially for those patients with limited capabilities.

While the previous clinical tests are mostly focused on the specific hand functions the Action Research Arm Test (ARAT) is a global arm function evaluation tool. Grasp, grip, pinch and gross movement are the four sub-scales within ARAT and each of them evaluate the abstract object manipulation strategies. Score is once again represented on a scale which this time ranges up to 57 corresponding to the normal upper limb function. The score is purely based on the subjective opinion of the certified examiner which can judge the quality of execution of each individual task on a scale from 0 (cannot perform) to 3 (performs normally).

Besides the four aforementioned tests, in order to target different upper limb functions and functional improvements while using assistive technologies, several other clinical tests have been developed. Some quite elaborate ones are the Assessment of Capacity for Myoelectric Control (ACMC) [79] and the Jebsen-Taylor Test of Hand Function (JTHF) [80]. The former is a clinical evaluation test specifically tailored for myocontrol and, although it suffers of a strong subjective component and it has not yet received wide recognition, may be a promising evaluation tool. 


\subsection{EMG as a gateway to the movement intention}

The EMG has been used as one of the sturdiest interfaces for neurorehabilitation technologies for over half of a century now [81]. It is an electrophysiological signal which is easy to access and it very well reflects the intention of a movement. So far the majority of applications were relying only on the global processing of EMGs in order to interpret the controls sent by the user. However, the EMG itself contains much higher volume of information on the neural drive which has so far been neglected for myocontrol purposes. In order to be able to extract this information and further apply it, basics of muscle physiology and EMG generation should be understood.

\subsubsection{Motor units and generation of EMG}

The motor unit (MU) provides the primary output for the central nervous systems and it translates sensory and descending neural information into forces which finally generate the movement [82]. As such, $M U$ is the basic functional unit of the neuromuscular system and it is comprised of a motor neuron along with its dendrites, axon and the corresponding muscle fibres [83]. Motor neurons are located in the spine and its axon projects in a peripheral nerve to the target muscle fibres which it innervates [84].

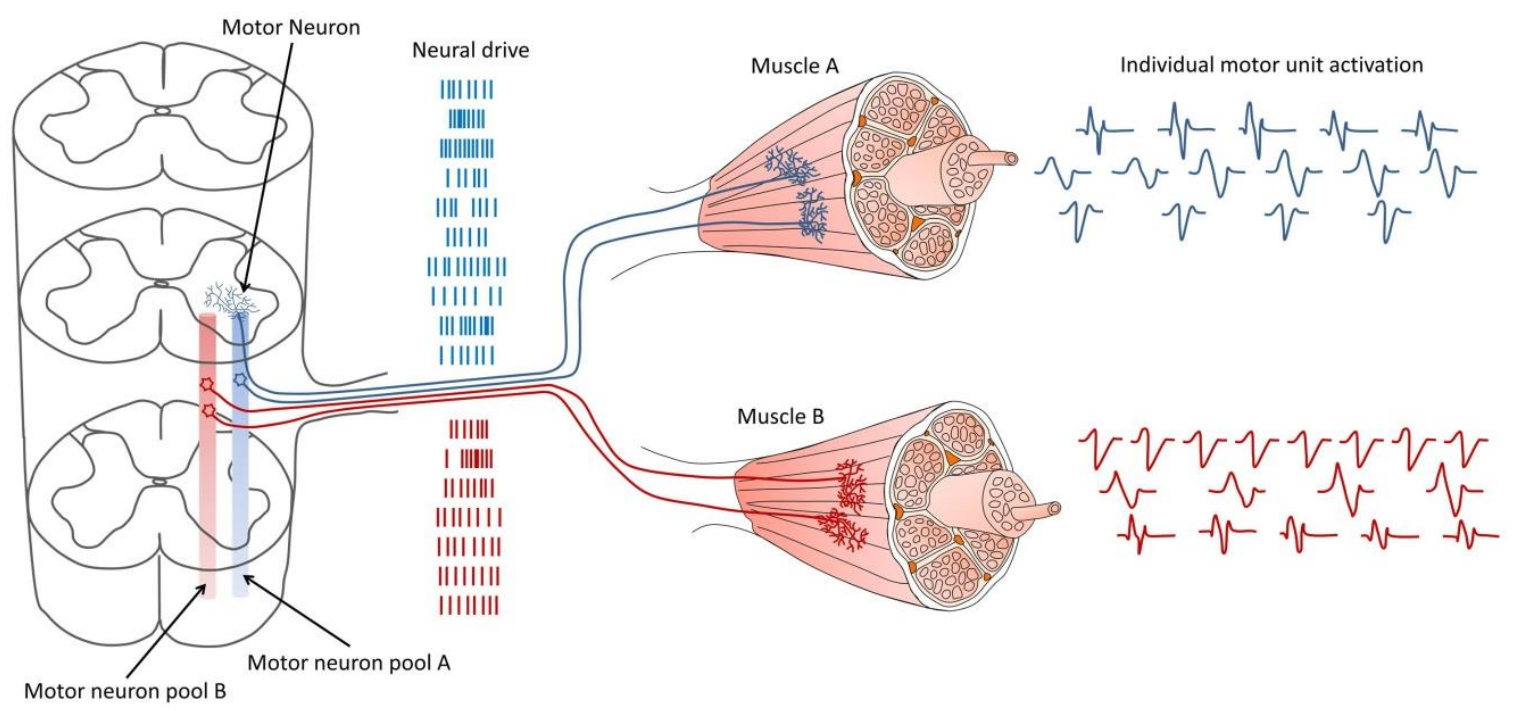

Figure 6 - Schematic representation of the descending motor pathway starting from the spinal cord (left) and terminating at the muscle (right). Main components of a motor unit are depicted including an exemplary sketch of detectable firings

Motor neuron pool, sometimes also referred to as motor nucleus, is a population of motor neurons that entirely innervate a single muscle [82]. A single motor neuron pool may be comprised of a few up to a couple hundred motor neurons [85], [86].

The number of muscle fibres innervated by a single axon of a motor neuron varies drastically across the motor neuron pool [84]. During a voluntary contraction first recruitment done is of those MUs which innervate fewer muscle fibres [82]. In general, 
majority of MUs in a muscle have smaller muscle units [87]-[89]. The MU territory represents the spatial distribution of all fibres belonging to a single MU [82]. The fibres of a single MU usually do not spread form one end to the other of a given muscle, but rather get terminated within the muscle fascicle [90], [91]. This ensures that the force generated by individual muscle fibres gets transmitted through various connective tissues during which it gets attenuated and the influence of different contractile properties ends up being reduced and having almost no effect on the generated motion [84].

Given the very conservative threshold for synaptic transmission at the neuromuscular junction during voluntary contractions, the motor neuron generated action potential invariably leads to propagation of action potentials along all the muscle fibres of a muscle unit at approximately the same time [92]-[95]. By placing the electrodes on the skin surface above the muscle, recording of the field potential generated by the summation of the extracellular currents comprised of sarcolemmal action potentials can be done [96]. Therefore, the resulting EMG provides the global measure of muscle activation as a result of the increasing number of motor units involved in an action [97].

\subsubsection{EMG decomposition}

As previously elaborated, there is a direct correspondence between the discharge of a motor neuron and MUAPs propagated by the innervated muscle fibres [84]. This means that the recorded EMG is a summation of the individual contributions of the currently active MUs. Therefore, by decomposing the EMG into its constitutive action potentials, an assessment of the neural drive to the muscle can be made.

The most straight forward way for identifying $\mathrm{MU}$ discharges is by acquiring an intramuscular EMG. Even though this technique is a common practice in clinical environment, it has significant drawbacks in neurorehabilitation applications. Therefore, numerous non-invasive methods have been proposed for indirectly assessing the neural drive.

Early algorithms suffered from the inability to deal with superimposition and interference between the MUAPs [98]-[101]. However, the more recent methods have managed to overcome this problem and to successfully identify even the complete $\mathrm{MU}$ discharge patterns [102]-[105]. These techniques are able to compensate for the variability in the shapes of the MUAPs by decomposing an interference signal into the discharge times of the MUs that contribute to the signal itself [82].

Through physiological modelling, according to [84] the surface EMG can be represented as a time-varying convolution of MUAP shapes: 


$$
\begin{gathered}
x_{i}(t)=\sum_{j=1}^{N} \sum_{k=1}^{K} \boldsymbol{h}_{i j, \tau_{j}(k)} * \delta\left(t-\tau_{j}(k)\right)+\omega_{i}(t), \\
i=1, \ldots, M ; t=1, \ldots, T
\end{gathered}
$$

where $*$ stands for convolution, $x_{i}(t)$ is the $i$-th surface EMG channel, $\omega_{i}(t)$ is an additive noise, $\delta(\cdot)$ is the unit-sample pulse and the $k$-th MUAP of the $j$-th MU appears at time $\tau_{j}(k)$. The MUAP observation $\boldsymbol{h}_{i j, \tau_{j}(k)}=\left[h_{i j, \tau_{j}(k)}(1) \cdots h_{i j, \tau_{j}(k)}(L)\right]$ varies in time, modelling all the temporal MUAP changes.

The described model enables for $\mathrm{MU}$ discharge patterns to be represented as binary time series ( 1 when MU discharge is present and 0 when it's not) even though MUAP shapes vary in space. This particular fact is then further exploited by some decomposition algorithms.

Changing muscle geometry and its influence to MUAP shapes can be neglected if an assumption of an isometric contraction is made. If in addition the observation interval is short enough not to allow muscle fatigue to appear, the MUAP shapes can be treated as stationary. This allows for EMG to be treated as linear, time-invariant, convolutive, multiple-input-multiple-output model where the $i$-th output is presented as [84]:

$$
x_{i}(t)=\sum_{j=1}^{N} \sum_{l=0}^{L-1} h_{i j}(l) s_{j}(t-l)+\omega_{i}(t), \quad i=1, \ldots, M ; t=1, \ldots, T
$$

where

$$
s_{j(t)}=\sum_{k=-\infty}^{\infty} \delta\left[t-\tau_{j}(k)\right], \quad j=1, \ldots, N
$$

For the simplicity of notation this EMG mixing process can be also stated in the matrix form:

$$
\boldsymbol{x}(t)=\boldsymbol{H} \overline{\boldsymbol{s}}(t)+\boldsymbol{\omega}(t)
$$

with $\boldsymbol{x}(t)=\left[x_{1}(t), \ldots, x_{M}(t)\right]^{T}$ being a vector of $M$ surface EMG channels, $\boldsymbol{\omega}(t)=$ $\left[\omega_{1}(t), \ldots, \omega_{M}(t)\right]^{T}$ representing an additive noise vector and $\overline{\boldsymbol{s}}(t)=\left[s_{1}(t), s_{1}(t-\right.$ $\left.1), \ldots, s_{1}(t-L+1), \ldots, s_{N}(t), \ldots, s_{N}(t-L+1)\right]^{T}$ standing for vectorised block of $\mathrm{L}$ samples from all the $\mathrm{MU}$ discharge patterns. $\boldsymbol{H}$ is a $M \times N L$ mixing matrix containing all the MUAP waveforms in ideal recording conditions.

In general decomposition techniques can be divided into template matching or blind source separation (BSS) approaches. The first ones are aiming to segment the EMG into recognizable waveforms, identify MUAP templates and match them together 
(perform clustering) [99], [104]. The latter algorithms are trying to directly estimate the mixing matrix $\boldsymbol{H}$ from the observations $\boldsymbol{x}(t)$ without introducing any a priori knowledge on either the mixing process or MU discharge patterns [84].

\subsection{The goal and the outline of the thesis}

The aim of this PhD project is to enhance the information extracted from the EMG signal for providing better clinical solutions for myocontrol than currently available in commercial and research-based systems. The approach applied include patients in all stages of the developments, as a way to effectively translate research efforts in solutions useful for the prosthetic users. This achievement provides a solid ground for development of a novel, more intuitive and effective generation of rehabilitation technologies. To achieve this goal, a set of techniques, combining engineering and clinical approaches, is proposed and evaluated. The main characteristics of the work done is a strong translational approach, so that all studies performed included patients with amputations, with a range of conditions, including very challenging clinical cases (e.g., transhumeral amputees or soft tissue injury patients).

The thesis includes three main studies, strongly linked to each other. In the first study, an evaluation of an advanced myocontrol algorithm in transradial amputees has been made and compared using different metrics for objective evaluation of performance. In the second study, the same advanced control has been applied to a very challenging clinical case - patients with severe soft tissues injuries who underwent elective amputations. Finally, the last study addresses another challenging clinical case, that of transhumeral amputees. These patients have undergone TMR and were treaded using the most advanced way of estimating the neural drive to muscle from the surface EMG, i.e. the direct estimation of the output discharges of the spinal cord circuitries.

Measuring the functional benefit of a novel neurorehabilitation technology is a difficult endeavour, and especially in the case of upper limb prosthetics. The user experience itself is influenced by numerous factors making it rather tough to determine whether the new myocontrol algorithm has potential in the real world applications or not. So far, academia has settled for using rather exact metrics derived from the machine learning community which delivers simple descriptive scores. However, a comparison presented here, between the most established offline scores and the clinically recognized tests, shows little to no correlation between the two, raising doubts on the validity of the former. This has also given a potential glimpse into where some of the reasons for such a strong dichotomy between academic and industrial solutions might be coming from. In addition, a revision of the most commonly used clinical evaluation tools has been suggested after analysing subject's kinematics during the execution of some of these tests. 
In the cases of severe soft tissue injuries a limb might remain present, but due to structural damage its function is most often highly compromised. Unfortunately, even with the most advanced reconstructive efforts, so far not much could have be done for these patients. Here, an extension of a bionic reconstruction method [106], involving elective amputation, advanced tech-neurorehabilitation and prosthetic fitting is presented and successfully tested on a case series of patients. The intention was to improve the functionality in everyday situation of the affected individuals. Through advanced signal processing and application of the machine learning algorithms presence of the full and intact neural drive is exploited for the purpose of delivering highly dexterous prosthetic control to this particularly difficult group of patients. Clinical evaluation shows clear improvement of functionality following this approach.

High level amputees suffer from the myocontrol paradox where the more distal amputations require articulation of larger number of joints, yet fewer muscle sources are available for sensing the desired control signals. Fortunately, Targeted Muscle Reinnervation (TMR) approach [11] is able to grant access to the full original neural drive by rerouting the remaining portions of the nerves initially hosted within the missing limb to the new target muscles in the proximity of the amputation. Even though significant, improvement achieved by simple one-to-one mapping between the newly reinnervated sources and joint functions of the prosthetic limb, is not exploiting the full neural information available after TMR. By decoding the activation properties MUs of the newly innervated muscle from the decomposed surface EMG, the neural drive information contained at the level of the spinal cord can be observed. Using this technique, data presented here suggests an improvement in the delivery of the proportional control signal compared to the standard approaches which could be of high significance for the new generation of the prosthetic technologies.

As an addition, two appendices are supplemented as a part of this thesis. The first one provides a technical note on the manufacturing process of specific sockets which have been particularly designed for the testing described throughout the monography. The second one refers to the details of the advanced myoelectric algorithm which has been used in the first two studies.

The main outcome of the thesis is a full clinical evaluation in a large variety of patients of an advanced simultaneous and proportional control algorithm and the proposal of a new way of interfacing patients through the identification of motor neuron discharges. The first algorithm has been developed and evaluated in this thesis at a level that it could be implemented in clinical devices. The second algorithm requires further steps for its full pre-clinical testing, but has been extensively tested in actual patients that could benefit from it. All cases presented in the thesis are related to challenging clinical conditions that are difficult to treat with current commercial solutions and that have been successfully addressed with the methods proposed in this thesis. 


\section{Performance assessment methods}

\section{This chapter is based on the work currently under revision:}

Vujaklija, I., Roche, A. D., Hasenoehrl, T., Sturma, A., Amsuess, S., Farina, D., et al. (2016). Translating Research on Myoelectric Control into Clinics - Are the Performance Assessment Methods Adequate? Front. Neurorobot.

Current gap between myoelectric solutions developed by academia and those that can be freely found on the market is substantial. This issue has been already raised [107], [108] and numerous attempts have been made in order to address it. Recently new control approaches have been introduced [54], [64], [67], different sensor types and sensor fusions were made [109]-[113], new surgical techniques have been developed [11], [106], and advanced hardware has been engineered [114]-[116]. However, all this progress hasn't still made a sizable step towards improving the experience of the everyday end user. Though, it is quite relevant for better understanding of the control problem at hand and its challenges. A common pattern which can be observed across all these studies is the general lack of tests performed on larger number of potential users. Additionally, most of the evaluations have been conducted in non-standardized scenarios.

The necessity for testing prosthetic solutions in a greater number of amputees than currently done is a widely recognized issue. Moreover, it is also evident that the tests used often fail to fully include clinically relevant metrics. Rather, performance metrics prevalent in laboratory research may be poorly associated to the clinical outcome [65], [70], [117]. Here, new insights and data are presented in order to further substantiate the relevance of this problem.

Transferring myoelectrical systems developed in the laboratory to clinical settings is a challenge that requires multidisciplinary efforts. Clinical tests, although not ideal, offer the most realistic prediction of the system performance in the daily use. These tests account for several of the challenges that laboratory-based assessment methodologies tend to neglect. For example, noiseless laboratory-based evaluation platforms fail to account for the end effector loads, poor socket fitting, and sweating.

Though, a down point of clinical tests is the fact that they only account for whether or not the subject is able to execute a certain task and in which time. Consideration for how the given task has been executed is either included through examiner's subjective opinion or it is not incorporated into the score at all. Here, in one subject, an amount of body compensation during the execution of several clinical tests has been observed using a motion tracking system. This is a proof of concept experiment which aims to show the necessity of this kind of evaluation during the testing of prosthetic performance. 
In this study, we review evaluation methods regularly applied for prosthetics use. Moreover, in Experiment 1 we provide data from seven conventional myoelectric users tested for prosthetic control ability with both classification accuracy and clinical assessments. Additionally, in Experiment 2 we provide a comparison of one patient using two types of control approaches against a pool of healthy volunteers performing same clinical tests, which supports the idea of having to extend the current clinical scores to account for body compensation in order to have the full performance evaluation of the given system.

\subsection{Methods and materials}

Seven male transradial amputees participated in this study. All of them are regular users of commercial myoelectric devices. For the purpose of the experiments they have all been fitted with a custom made sockets (Appendix I) and the Michelangelo hand (Ottobock Healthcare $\mathrm{GmbH}$, Austria) with additional rotation and flexion/extension unit. All users controlled the aforementioned advanced prosthesis using an algorithm based on the common spatial pattern (CSP) based classifier, as described by [67] (Appendix II). The EMG signals were recorded with 8 bipolar surface electrodes (Otto Bock raw signal electrodes $13 \mathrm{E} 200=50 \mathrm{AC}$ ). The control system allowed the subjects to access seven prosthetic functions - wrist flexion/extension, wrist pronation/supination, hand open, pinch, and key grip.
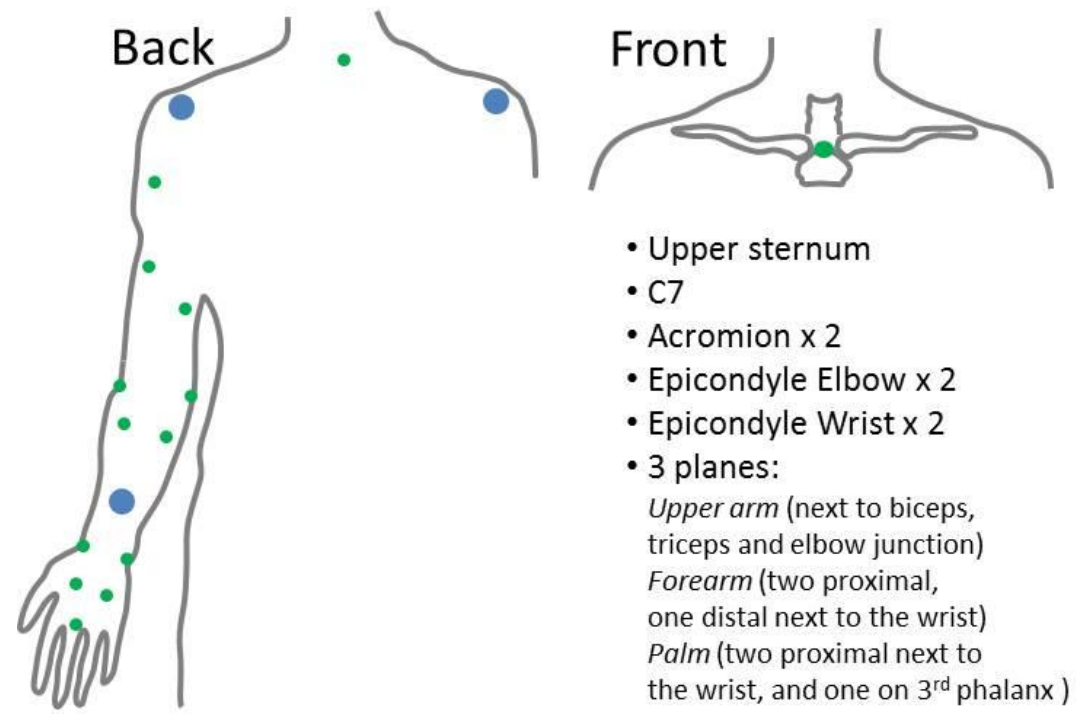

Figure 7 - Position of passive reflective markers used for motion tracking during the performance evaluation. $10 \mathrm{~mm}$ markers are depicted in green and $20 \mathrm{~mm}$ ones in blue.

In order to accurately analyse the kinematics of the upper limb and trunk during prosthesis usage, 17 passive reflective markers (diameter: $10 \mathrm{~mm}$, except for 3 with diameter $20 \mathrm{~mm}$ ) were secured to the dorsum of the participant's left arm at welldefined anatomical positions to clearly identify all movements made by the arm (Figure 7). Reference markers were positioned above C7, sternal angle, and the 
acromion processes. Clusters of markers were used to define the centroids of four segments of interest - palm, lower arm, upper arm and thorax. An eight-camera VICON MX+ optoelectronic motion capture system (Oxford Metrics Ltd., Oxford, UK) was used to capture the movements of the passive markers at a sampling rate of 200 $\mathrm{Hz}$. The coordinates of the markers were recorded in 3D-space in relation to time. The motion data were processed using MATLAB 2013a (Mathworks, Massachusetts, USA). For assessing the movements of the upper limb in relation to natural movements, five able-bodied male subjects were also measured as a normative group.

The whole study has been was performed in accordance with the recommendations of the local ethics board of the Medical University of Vienna (Ethics Commission number 1044/2015), with written informed consent from all subjects. All given consents are in accordance with the Declaration of Helsinki.

\subsubsection{Experiment 1}

Purpose of Experiment 1 was to test the correlation between standardized offline myoelectic control assessment score and two clinical tests - of BBT and SHAP. We provide data on amputees that compare the accuracy estimated offline, for one of the classic control schemes developed over the past decades, with clinical scores. These data serve the purpose of reinforcing the conclusion for the need of clinical tests in an exemplary way. Therefore, the experiment and results do not aim at providing general conclusions on all myocontrol schemes and evaluation methods but rather support the view presented in this perspective. All patients were asked to mount their custom made sockets with the advanced prosthetic system in place. They were prompted to perform the following hand and wrist motions: wrist flexion/extension, wrist pronation/supination, hand open, lateral and pinch grip. All the motions were recorded in three arm positions (relaxed, fully extend arm in front of the ipsilateral shoulder and fully extended arm across the contralateral shoulder) and at three force levels (30\%, 60\% and $90 \%$ of EMG based maximum voluntary contraction for each motion). For offline accuracy assessment, the classifier was trained by data collected in only one arm position and tested against the remaining two data sub-sets. The average of the three scores was the reference performance of the subject. The entire data set was used for training the same CSP classifier that allowed execution of BBT and SHAP tests. These particular clinical tests have been representatively chosen since they cover a wide range of assessment goals while being entirely objective. Additionally, these two tests have been widely recognized and familiar to academic and industry-based developers as well as clinical experts. 


\subsubsection{Experiment 2}

Experiment 2 was designed in order to assess the compensatory movements of a patient while performing CPRT and SHAPT tests. Besides the previously described fitting (Figure 14), the patients were instructed to use his regular device as well. This system was equipped with the classic control algorithm for hand closing, opening and rotation of the Michelangelo hand that he used for more than 18 months and which allowed robust control during the activities of daily living. This fitting enabled him to use the prosthesis in daily activities quickly after the elective amputation.

Upon finishing the Experiment 1, selected patient underwent further training to optimize his control of these additional DoFs using both proportional and simultaneous movements at the wrist (Figure 14). After a short training period of 2 weeks, the patient completed the SHAP and CPRT with the advanced prosthesis and control algorithm for comparison with his traditional device. During this execution the patient's upper body movements have been monitored using the aforementioned camera setup.

Five able bodied subject which participated in this experiment in order to form a normative group were also instructed to complete SHAP and CPRT. Their kinematics was as well monitored for the whole duration of the experiment.

\subsection{Results and discussion}

\subsubsection{Experiment 1}

The performance scores in both offline and clinical tests are presented in Figure 8 . The offline classification accuracies are slightly lower than in other studies [118], [119] presumably because of the different arm positions used for training and testing as well as the full prosthetic fitting which is not usual in offline evaluation studies. Although with these choices we have maximized the prediction capacity of offline indexes for clinical scores, still the clinical scores did not strongly correlate with the offline performance measures. The clinical scores had poor correlation with the offline performance measures. For example, there were two patients who achieved a similar SHAP score just below 40 whereas they showed substantially different classification accuracies of $<70 \%$ and $>85 \%$ (Figure $8 \mathrm{~A}$ ). Similarly, two patients who had very similar classification accuracies of $70-75 \%$ had SHAP scores of 27 and 47 (Figure 8A). The BBT requires less skill to be performed than the SHAP. However, the BBT score was even less associated to the offline classification than the SHAP was (Figure 8B). For example, subjects with an offline accuracy of over $95 \%$ performed very differently in this test (Figure 8B). 

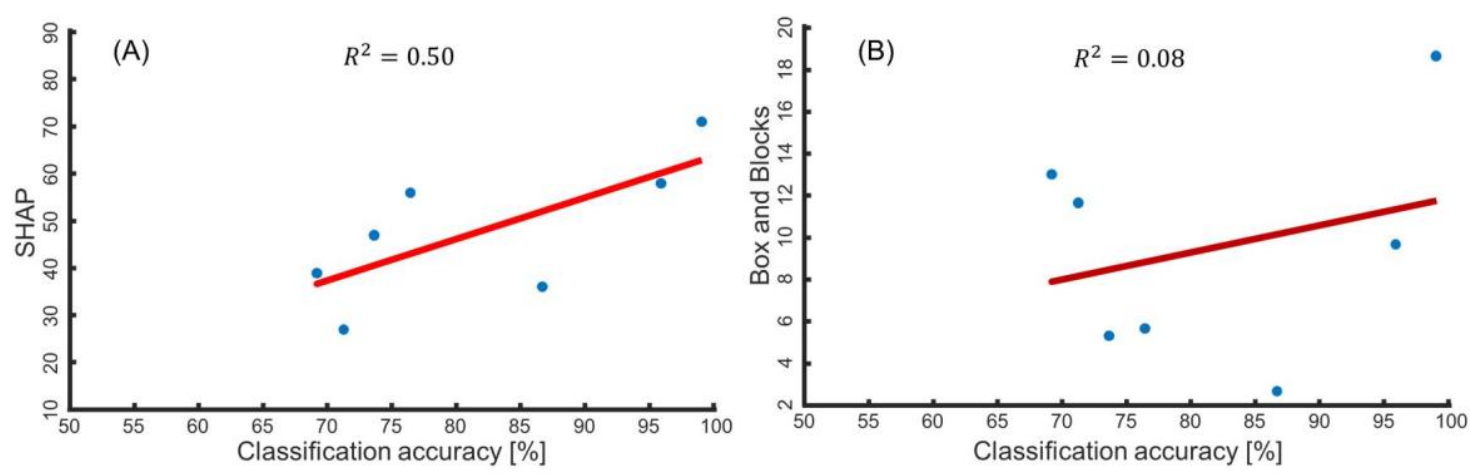

Figure 8 - (A) The correlation between the clinical SHAP score and the offline classification accuracy indicates a weak association. The offline scores have been obtained in realistic conditions with the patients wearing their prostheses and training and testing performed on sets of data obtained in different arm positions. Despite the realistic conditions, the associations shown here are not strong. For example, a SHAP score of approximately 40 may correspond to classification accuracy lower than $70 \%$ or greater than $85 \%$ depending on the user. The SHAP requires precise manipulation over short periods of time which is not captured by this offline metrics. (B) The correlation between the clinical Box and Blocks test and the offline classification accuracy shows almost complete absence of association between the two. For instance, the two patients who achieved the classification accuracies $>95 \%$ were radically different $\mathrm{n}$ the number of blocks they could transfer.

Furthermore, by considering strictly the hand movements - hand open, fine pinch and key grip - that are primarily used for this test, the mismatch between this test and offline performance is even more substantial (Figure 9). For the two subjects representatively considered in Figure 9, the average classification rate across the three hand motions was $89 \%$ and $79 \%$ whereas the transferred blocks (score of the BBT) were 5 and 12, respectively.

When the offline evaluation was performed by using data collected without wearing the prosthesis and tested on the same arm position as the training, as more commonly done in laboratory tests (e.g., [120]-[123]), the resulting offline classification rates were high and comparable to those reported in the literature ( $>90 \%$ on average). However, once fully fitted, the majority of patients were unable to successfully conclude the clinical evaluations without retraining, indicating that the classic offline evaluation procedure performed in several research studies does not provide strongly relevant clinical information. 

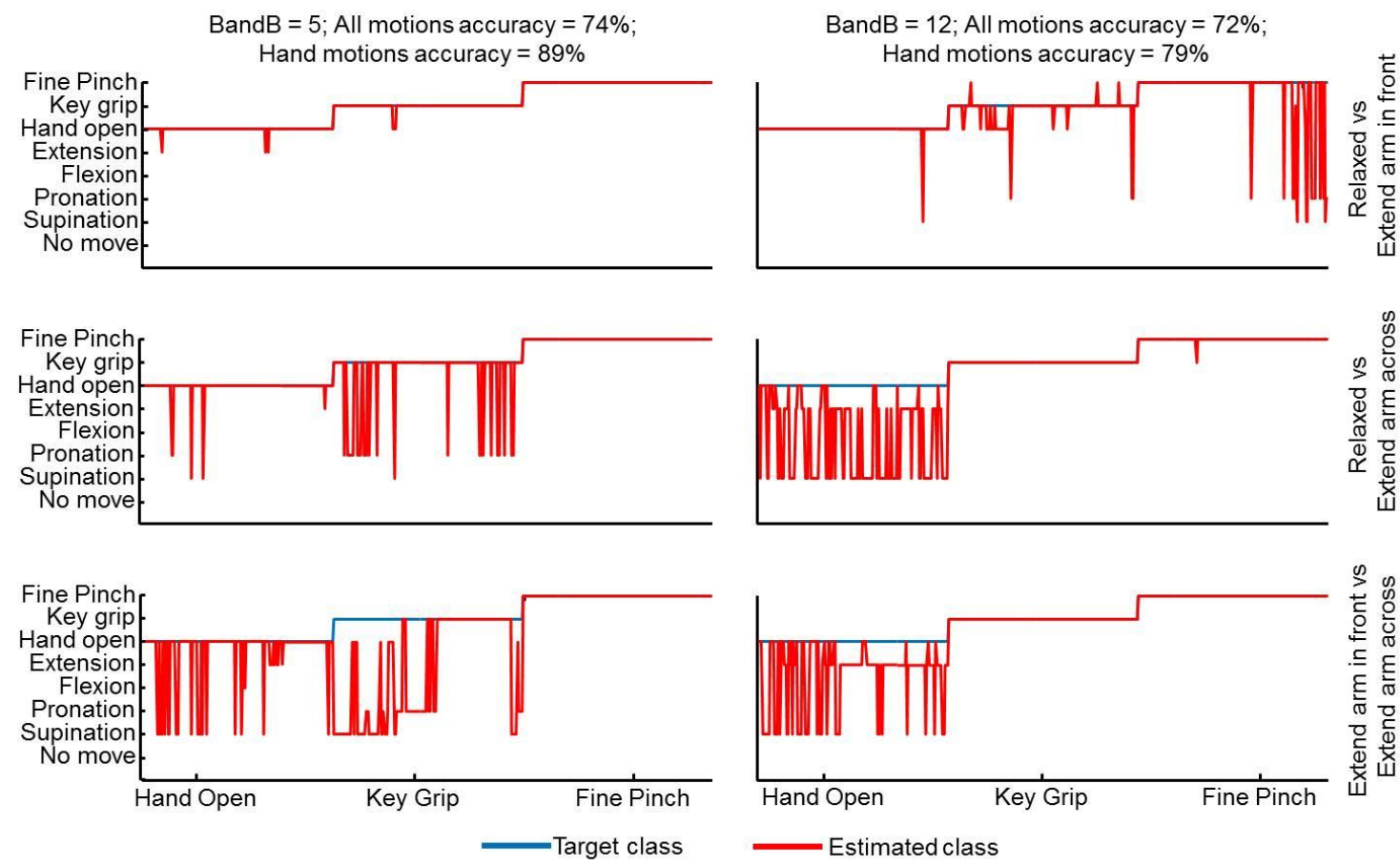

Figure 9 - Classification output for two patients with substantially different Box and Blocks outcomes but very similar classification accuracies over all motions. The focus is here on the three hand motions that are most relevant for the Box and Blocks task - hand open, key grip and fine pinch. The offline accuracy for these motions is lower for the subject with the higher clinical score.

\subsubsection{Experiment 2}

The control capabilities of the patient, as revealed by the SHAP, were similar for the classic (score of 83), and the advanced prostheses (score of 68 ). This was achieved even if the subject had trained substantially less time with the advanced control with respect to the traditional prosthesis. Moreover, the observation of the movements of this patient during the standardized tests clearly indicated that he could perform more natural tasks with the advanced prosthesis (Figure 10 and Figure 11). The ability of the adapted prosthesis to provide additional DoFs, and the intuitive control algorithm to make use of these motions, was judged subjectively as very important by the patient, although it was not quantified by the SHAP, which is not designed to assess dexterity but only the time needed to complete each sub-task. 


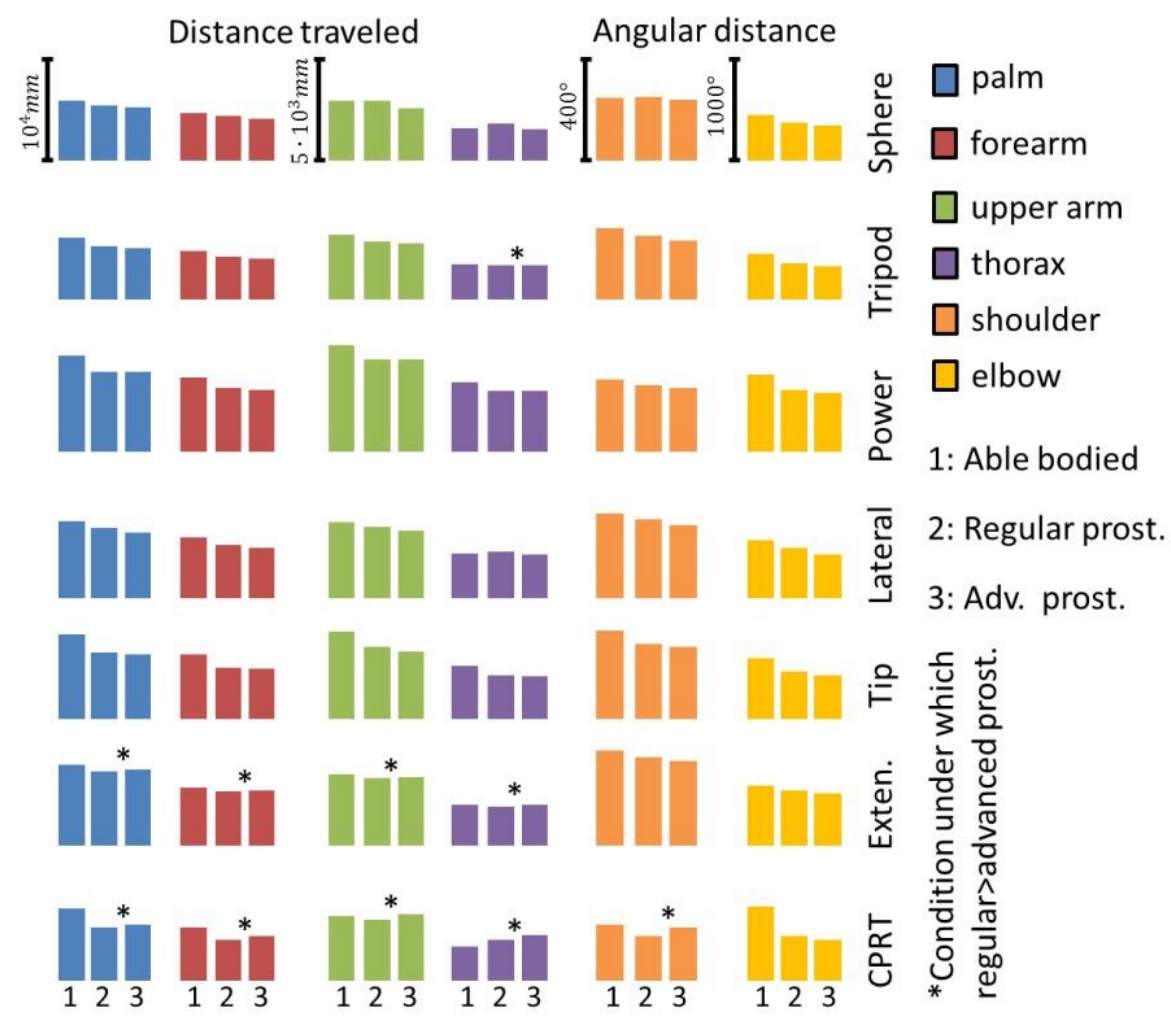

Figure 10 - Recorded kinematics with respect to anatomical segments and joints across different sub-groups of SHAP test and CPRT for able-bodied group (1), Patient 1 with classical prosthesis (2) and Patient 1 with advanced prosthesis (3). Notably, in terms of kinematics, Patient 1 was more efficient during the execution of tasks than on average all five able-bodied participants.

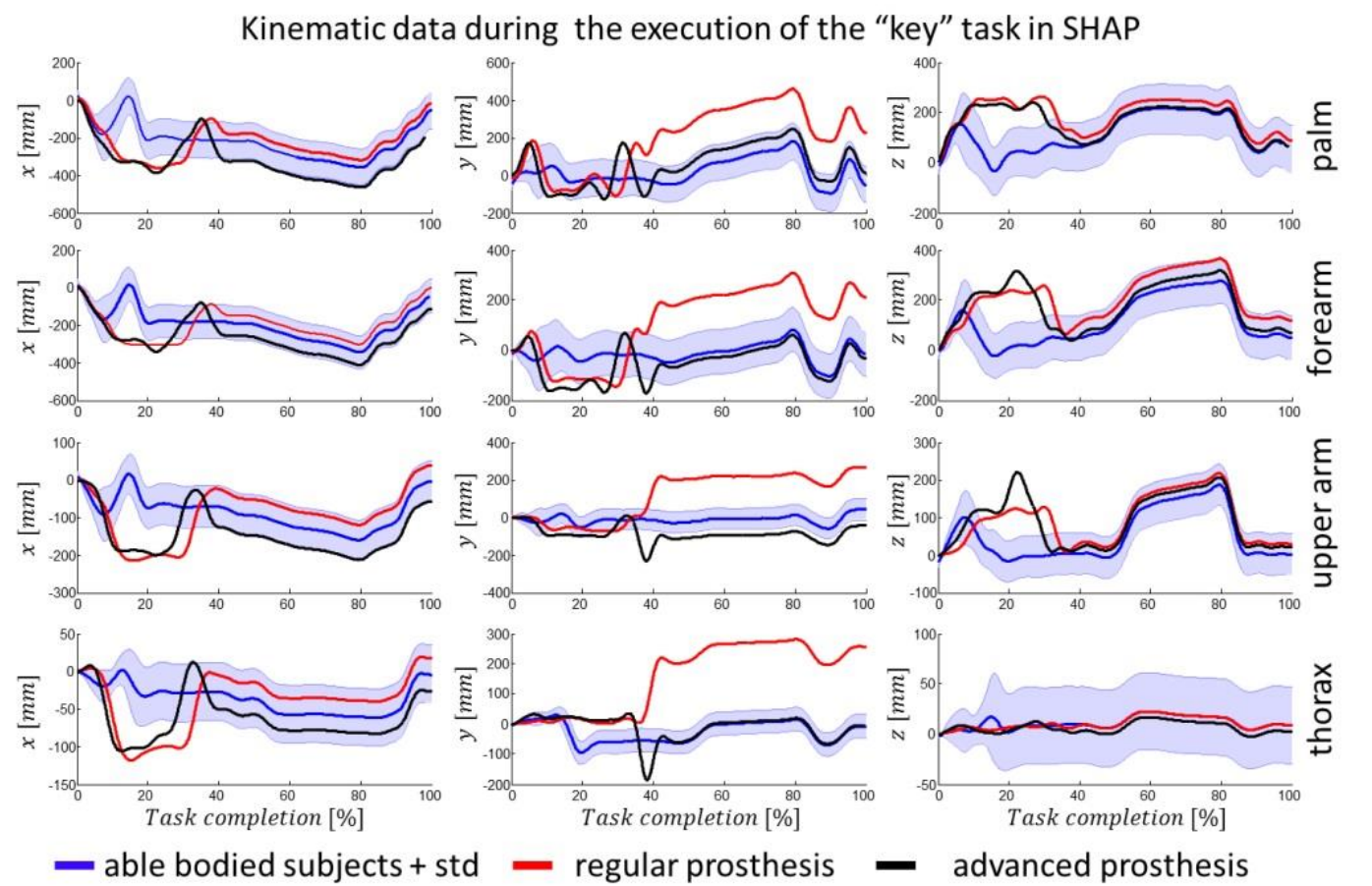

Figure 11 - Recorded centroid traces of respective anatomical sections across all three axes during the execution of an example task of the SHAP test, the key task, for able bodied group, patient 1 with classical prosthesis and patient 1 with advanced prosthesis 


\subsection{Conclusion}

Abandonment rates among upper limb myoelectric prosthetic users are still very high [38], [40], [41]. At the same time, research efforts have provided several new solutions for myocontrol that have been proven to be highly functional under strictly laboratory conditions. The negligible transfer from research to real world applications likely depends, as one of the most relevant factors, on an insufficient level of evaluation procedures.

Using novel prototypes of myoelectric systems in daily life would provide the ultimate assessment but this strategy would neither be safe nor always legal. Therefore, clinical evaluations are a compromise between laboratory conditions and real-life tests. Although not perfect, clinical tests are closer to the conditions of interest for the users than offline assessments or online tests using virtual prostheses. The results presented in this study indicate a poor association between common research scores and clinical ones, which may justify the gap between research prototypes and commercial products.

Considering the discrepancy presented in the literature and supported here with new data, it is imperative that novel myoelectric systems are fully clinically evaluated when assessing their performance. In addition, clinical test either should not be fully taken for granted. As data presented in Experiment 2 suggests, not all aspects of the user and system performance are accounted for in the current clinical evaluations. Even though the scores might be high, the way they have been achieved might not be the most natural one. This, if not straight away, then maybe later during the everyday use, might result in overuse of compensatory joints and finally system abandonment.

For this reason, researchers and clinicians should jointly devise a standardized testing framework for quantitatively and qualitatively assessing the performance of upper limb prosthetic devices and their users. 


\section{Bionic reconstruction enables dexterous prosthetic control after elective amputation for critical soft tissue injuries}

\section{This chapter has been based on the recently published paper:}

Aszmann, O. C., Vujaklija, I., Roche, A. D., Salminger, S., Herceg, M., Sturma, A., et al. (2016). Elective amputation and bionic substitution restore functional hand use after critical soft tissue injuries. Nat. Sci. Reports.

As previously mentioned hands are an essential part of our everyday interaction with the outside world. Though, exactly this puts them into harm's way at all times. It has been reported that as much as a third of all work-related injuries are involving upper limbs, out of which $30 \%$ either involve a crushing mechanism, fracture or amputation [124]. Traumatic events, such as high voltage electrocution, crush, or degloving injuries can indeed be devastating for a limb [23], [125]-[127]. Therefore, it is of outmost surgical importance that the limb and its function are preserved to the greatest extent possible [25], [128], [129]. Until now, a battery of reconstruction techniques has been developed and described in literature [25], [129].

Biological reconstruction techniques will always be the ones attempted first, but they can be successful only until a certain degree. In severe cases of critical tissue loss, functional recovery is not always possible, resulting in a limb that is not only useless but may also constitute an impediment to the patient [23], [130]-[133]. In cases where blood supply to the arm is transiently interrupted with ischemia and reperfusion injury, similar outcomes can occur leading to established Volkmann's contractures [134]. It is known that radiation treatment in oncological cases can cause the loss of entire compartments resulting in a useless hand [135]. Considering the severity of these complications, resorting to advanced prosthetic technology may be the only option for restoring some functionality of the limb.

Bionic reconstruction has recently been demonstrated to provide useful hand function in patients with global plexopathies with multiple root avulsion injuries [106]. In those patients, prosthetic control was challenged by damaged peripheral nerves, which limited the quality and quantity of electromyographic (EMG) signals that the patients could generate for control. Similar to the patients with damage to peripheral nerves, patients who have suffered critical soft tissue defects are also left with a stiff, insensate hand that is beyond surgical reconstruction. However, unlike the peripheral nerve patients, the remaining muscles have been critically damaged limiting them as sources for existing EMG based prosthetic control [136].

As previously indicated, nowadays of the shelf myoelectric prostheses are able to deliver simple proportional control over 1 or 2 DoFs. Even though robust, limitations of these systems are obvious and have been previously elaborated. In order to expand 
the possibilities of prosthetic control, recently methods suited for the dexterous control of multiple DoFs using the residual EMG activity have been developed [67], [136]. This system focuses on movements of the wrist and hand in order to provide a high gain in function, while preserving the robustness of control, needed for reliable daily use.

This chapter reports the concept of elective amputation and prosthetic replacement in three patients with critical soft tissue injuries who, despite all attempted biological reconstructive efforts, could not regain any useful hand function years after the injury. This is the first attempt of bionic reconstruction in this group of patients, and it is based on a recent report of this intervention after global brachial plexus injuries with multiple root avulsions [106]. Therefore, the versatility of this concept is confirmed by expanding the patient group to those with critical soft tissue defects to gain simultaneous and proportional prosthetic hand control.

\subsection{Results}

\subsubsection{Standardized Functional Outcome Measurements}

In three patients who had suffered critical soft tissue defects (Figure 12), prosthetic hand function was measured both before and after bionic reconstruction using standardized functional outcome measurements. Clinical test selected were the Action Research Arm Test (ARAT), the Southampton Hand Assessment Procedure (SHAP), and the Disability of the Shoulder, Arm, and Hand (DASH) questionnaire [78], [137], [138]. All three patients were fitted with commercially available prosthetic hands.
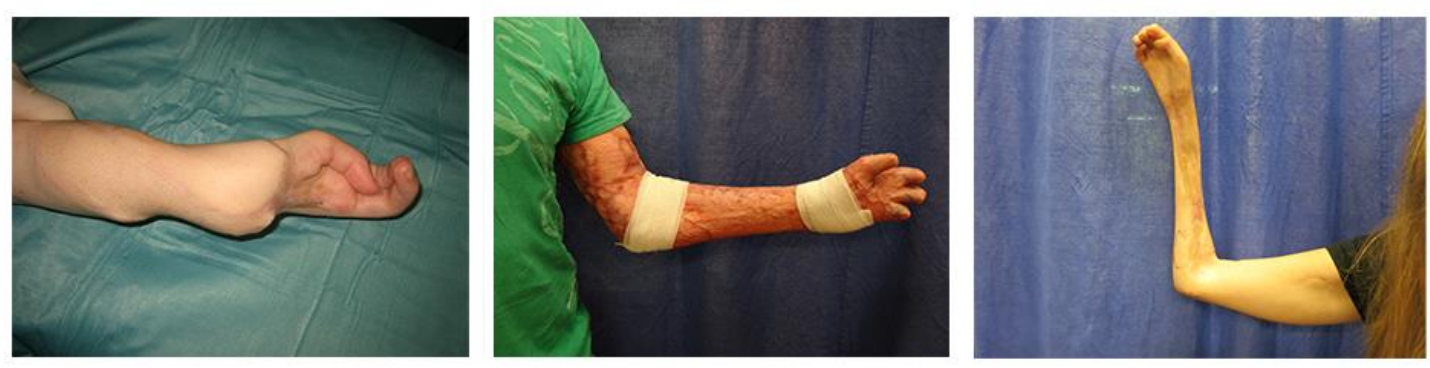

Figure 12 - The critical soft tissue injuries suffered by the patients in this study were due to (left) electrocution, (centre) degloving injury, and (right) complications secondary to compartment syndrome. In all cases the reconstructive surgical ladder was attempted first, but with poor functional outcome.

Pre-interventional testing confirmed absence of any sensory and motor hand function in all patients (Figure 13). All participants stated that they neglected the use of the impaired hand in daily life. Once specifically asked to perform bimanual tasks, use of impaired limbs was reduced to minimum. Post-interventional testing took place at least three months after prosthetic fitting, except in the case of Patient 3 who was evaluated at 10 days after, before she returned to her home country. 

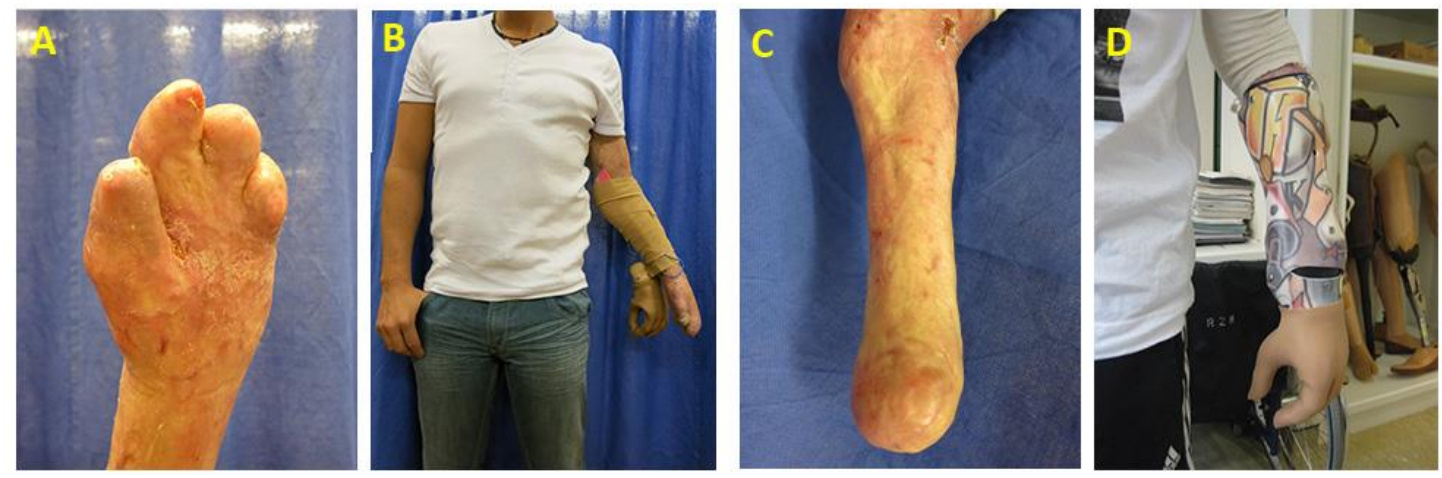

Figure 13 - Patient 2, (A) before bionic reconstruction, (B) during hybrid hand training, (C) healed residual limb after elective amputation, and (D) final prosthetic fitting with patient's own customized socket design and art.

Functional outcomes are documented in Table II. Across all patients, the DASH outcome scores improved from a mean of $40.4 \pm 19.7$ to $14.5 \pm 10.6$, the mean ARAT score also improved from $7.67 \pm 4.16$ during intermediary testing with a hybrid hand to $21.0 \pm 4.36$, and, after final prosthetic fitting, to $36.0 \pm 6.00$. This trend of gradual improvement was also observed during SHAP testing. Before the intervention, the patients scored a mean of $12.0 \pm 3.61$, with hybrid fitting $28.7 \pm 2.89$, and, after final fitting, $60.0 \pm 29.3$. Patient 3 was unable to attend the 3 -month follow-up as she lived in another country, thus her final reported SHAP score was measured 10 days after the final fitting.

Table II - Functional outcome scores for all critical soft tissue patients before (Pre) and after (Post) bionic reconstruction

\begin{tabular}{|c|cc|ccc|ccc|}
\cline { 2 - 9 } & \multicolumn{2}{|c|}{ DASH } & \multicolumn{3}{c|}{ ARAT } & \multicolumn{3}{c|}{ SHAP } \\
\cline { 2 - 9 } & Pre & Post & Pre & Hybrid & Post & Pre & Hybrid & Post \\
\hline \hline Patient 1 & $62 \cdot 0$ & 7.5 & 9 & 24 & 42 & 11 & 27 & 83 \\
Patient 2 & 23.33 & 9.17 & 11 & 23 & 36 & 16 & 32 & 70 \\
Patient 3 & 35.83 & 26.67 & 3 & 16 & $30 *$ & 9 & 27 & $27 *$ \\
Mean & 40.39 & 14.45 & 7.67 & 21 & 36 & 12 & 28.67 & 60 \\
Standard Deviation & 19.73 & 10.62 & 4.16 & 4.36 & 6 & 3.61 & 2.89 & 29.31 \\
\hline
\end{tabular}

Notes: DASH - Lower scores represents better function. In both the ARAT \& SHAP higher scores represents better function. Normal hand function is regarded as equal to or above 100 points in the SHAP. *Note Patient 2 was evaluated at 10 days after prosthetic fitting, and as lives in a separate country was unavailable for further follow up by our group.

\subsubsection{Assessment of Simultaneous, Proportional \& Dexterous Control}

Patient 1 was first fitted with a classic control algorithm for hand closing, opening and rotation that he used for more than 18 months and that allowed robust control during the activities of daily living. This fitting allowed him to use the prosthesis in daily activities quickly after the elective amputation. However, from the EMG signals recorded from this patient, it was evident that he could likely control additional movements with respect to closing, opening and rotation of the prosthetic hand, in a 
more natural way. Therefore, a customized Michaelangelo prosthetic hand that included wrist flexion, extension and rotation in addition to two grasps (palmar and lateral), was developed and custom-fit to the patient. The specific socket capable of hosting the prosthesis has been developed for this particular purpose (Appendix I). He then underwent further training to optimize his control of these additional DoFs using both proportional and simultaneous movements at the wrist (Figure 14). After a short training period of 2 weeks, the patient completed the SHAP with the advanced prosthesis and control algorithm (Appendix II) for comparison with his traditional device. The control capabilities, as revealed by the SHAP, were similar for the classic and advanced prostheses (SHAP score of 68), even if he had trained substantially less time with the advanced control with respect to the traditional prosthesis.

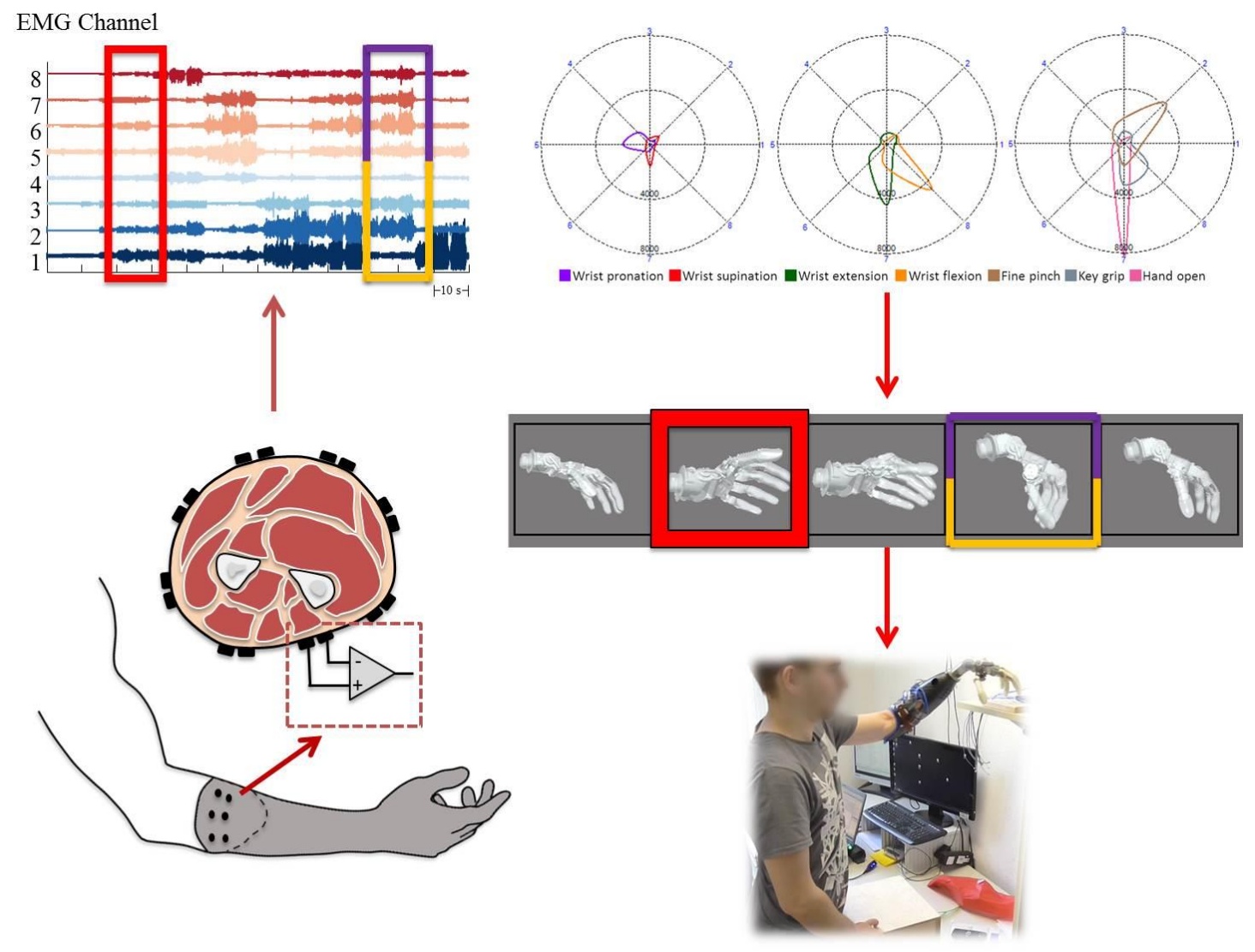

Figure 14 - Schematic of the patient training to achieve proportional and simultaneous control at the level of the wrist. First the patient's EMG activity is recorded using eight equidistantly placed surface electrodes during a calibration phase. The gross EMG signal is then decomposed into specific patterns that correspond to seven actions of the prosthetic hand, plus a resting condition. These patterns are uploaded to the prosthetic hand for real time control, which allows for both proportional and simultaneous movements of prosthesis in real-world situations. Details of the used algorithm are describe in Appendix II. 


\subsubsection{Pain \& Quality of Life}

10-point Visual Analogue Scale (VAS) was used to evaluate pain scores relevant to the affected limb. Prior to bionic reconstruction, patients 1, 2, and 3 reported pain scores of $0,0.5$, and 1.5 , respectively. No pain was reported by any of them after the bionic reconstruction.

Changes of quality of life, after bionic reconstruction, were assessed by SF-36 Health Survey (German Version, 4-week recall) [139]. Evaluated sub-items and summary scales are documented in Table III. Upon final prosthetic fitting and restored ability to perform bimanual tasks, a marked improvement of physical functioning was noted in all patients. Overall, bodily pain was successfully reduced in all three patients, exhibiting optimized social and emotional role functioning. Additionally, mental health was enhanced. General health perception improved in Patient 1 and 2, implying the importance of functional recovery in these patients. This score remained the same in Patient 3.

Table III - Quality of life rated for the three patients before (Pre) and after (Post) bionic reconstruction, evaluated with the SF 36 (rated from 0-100, where 0 represents the poorest quality of life, and 100 the best)

\begin{tabular}{|c|c|c|c|c|c|c|}
\cline { 2 - 7 } \multicolumn{1}{c|}{} & \multicolumn{2}{c|}{ Patient 1 } & \multicolumn{2}{c|}{ Patient 2 } & \multicolumn{2}{c|}{ Patient 3 } \\
\cline { 2 - 7 } \multicolumn{1}{c|}{} & Pre & Post & Pre & Post & Pre & Post \\
\hline Physical functioning & 75 & 95 & 70 & 95 & 85 & 90 \\
\hline Physical role functioning & 100 & 100 & 0 & 25 & 100 & 100 \\
\hline Bodily pain & 84 & 100 & 84 & 100 & 84 & 74 \\
\hline General health perception & 87 & 100 & 72 & 82 & 72 & 67 \\
\hline Vitality & 60 & 50 & 75 & 70 & 80 & 90 \\
\hline Social role functioning & 100 & 100 & 87.5 & 100 & 100 & 87.5 \\
\hline Emotional role functioning & 100 & 100 & 33.3 & 66.7 & 100 & 100 \\
\hline $\begin{array}{c}\text { Mental health } \\
\text { Physical component summary } \\
\text { scale }\end{array}$ & 84 & 88 & 68 & 72 & 84 & 96 \\
\hline $\begin{array}{c}\text { Mental component summary } \\
\text { scale }\end{array}$ & 50.9 & 57.7 & 43.8 & 51.9 & 51.2 & 48.8 \\
\hline
\end{tabular}

All patients reported improvement of social engagement and in general higher functionality during activities of daily living. Interaction with their environment was simplified and physical appearance with regards to self-confidence got enhanced. Following the prosthetic replacement, Patient 2 was able to continue working as a manual labourer, a wish he had long given up before bionic reconstruction offered him motor recovery of his injured hand. Patient 1 was able to return to work as an electrician after receiving treatment. 


\subsection{Discussion}

Study presented in this chapter reports successful restoration of hand function following bionic reconstruction in three patients which have suffered critical soft tissue injuries. This procedure was undertaken only after all established biological reconstruction techniques have previously failed. The success was achieved through a combination of surgical reconstruction, structured rehabilitation training, sophisticated signal extraction, carefully planned elective amputation, and advanced prosthetic systems.

Functional recovery following mutilating hand injury depends on a variety of factors, each of which might have impeding consequences [140]. Multiple level injuries such as crush with subsequent tissue ischemia, or massive loss of functional tissue are the ones leading to the worst functional outcomes [141]. The subject pool presented in this study comprised exactly out of these patients who have suffered devastating injuries, either due to electrocution, degloving or compartment syndrome. All of them have extended even far beyond the hand, affecting the limb at multiple levels, and are as such representative of the difficult cases that confront reconstructive teams. As later thoroughly explained, all biological reconstructive means were first attempted to restore function, yet the functional outcomes were non-existent. As there were no other autologous means that could be attempted in these patients, bionic reconstruction was offered to them as the last resort. This chapter demonstrated that the restoration of hand function in patients with critical soft tissue injuries is achievable with this multidisciplinary reconstructive approach.

Muscles, or more precisely EMG signals, were used as the interface for prosthetic control in this study. Property of muscles to act as translators of intuitive neural information and biological amplifiers of nerve activity was extensively relied on. Good prosthetic control depends heavily on consistency, accuracy, intuitiveness, function, and, above all, robustness over a broad range of conditions, including activities of daily living. Despite important developments in brain and nerve interfacing [142], [143], muscle interfacing is the only current viable way for daily use of prosthetic systems. With this in mind, it is understandable why critical soft tissue defects represent a particular challenge for applying neurorehabilitation technologies. The technique we have developed utilizes what soft tissue is left, and uses the gross EMG signal for myoelectric control, without the need for invasive interfaces [144].

All patients in this study have been fitted with customized commercially available devices which included the standardly available myocontrol. In addition to it, in order to further explore the possibilities of the presented approach, an advanced control algorithm, previously tested in realistic conditions by transradial amputees [52], has been adapted for use in one of the presented patients. Relaying on this algorithm, in comparison to the industrial state of the art in myocontrol, patient had an advantage 
of controlling multiple DoF concurrently, which corresponds to natural movements, in an intuitive way (without switches or similar mechanisms). It has been shown that a patient undergoing bionic reconstruction following elective amputation is able to control 3.5 DoFs in a dexterous way.

All the results present in this study reflect situations closely resembling daily activities, with an array of tests that quantified in depth their functional capacity. Moreover, all patients used the prosthetic systems they received in this study at home and during work. In this way evidence of the real functional gain achieved by bionic reconstruction was made available, and is the direct basis for translating research outcomes into clinical systems for daily use. Indeed, the three patients currently use their prostheses on a daily basis for an average of 6 to 14 hours per day.

Besides the severe motor impairment, major upper limb injuries are not only physically devastating, but also contribute greatly to psychological harm, leading to anxiety and depression [145], [146]. If biological reconstruction fails, as in the three patients described in the present study, the patients are confronted with the prospect of life with a useless limb. Beyond impairment in daily life this also reflects their self-image and self-worth [147]. Addressing the psychological impact of mutilating hand injuries is likely to improve functional outcomes [148]. In relation to failure of limb salvage, amputation has been shown to have no disadvantage from a psychological perspective in critical soft tissue defects [149], and wearing prosthesis helps amputees to maintain a body schema in which the missing limb is matched to the prosthesis [150]. Therefore, it can be stated that replacing a non-functioning limb with a prosthetic one besides functional gain, can also be psychologically rewarding. This has been confirmed in this study, by the improvement in quality of life metrics in all patients.

In cases where biological reconstruction was not successful, as those shown here, it is imperative that solutions outside the biological arena are considered and offered to patients. As has been previously demonstrated, the careful application of bionic systems can replace hand function in neurological injuries [106]. The same philosophy was applied in this study to three patients with critical soft tissue injuries, with different challenges with respect to our previous report. The surgical procedures and rehabilitation program were similar to those used for neurological injuries, albeit for different indications, but with similar successful results in terms of recovered function. Moreover, intact efferent pathways allowed the demonstration of highly dexterous control (3.5 DoF simultaneously and proportionally controlled) in one of the patients treated. We foresee that the two remaining patients will also be able to use this advanced control system after appropriate training. The technique of bionic reconstruction is a clinical reality and not a laboratory-based concept, and institutions with similar resources and skill could apply this method to patients with similar devastating injuries. 


\subsection{Materials and Methods}

\subsubsection{Study population}

Three patients (Table IV), who had suffered critical bone and soft tissue defects, participated in this study. They have read and signed informed consents between January 2010 and November 2014. Ethical approval was obtained from the Medical University of Vienna's Institutional Human-Studies Review Board (Ethic Commission Number 1209/2012).

Table IV - Characteristics of three patients who have suffered critical soft tissue injuries and have participated in this study

\begin{tabular}{|c|c|c|c|c|c|c|}
\hline Patient & Sex & $\begin{array}{c}\text { Age at } \\
\text { amputation }\end{array}$ & Mode of Injury & $\begin{array}{c}\text { Time between } \\
\text { Injury \& } \\
\text { Amputation }\end{array}$ & $\begin{array}{c}\text { Original } \\
\text { Dominant } \\
\text { Handedness }\end{array}$ & $\begin{array}{c}\text { Side of } \\
\text { Injury }\end{array}$ \\
\hline \hline 1 & Male & 23 & $\begin{array}{c}\text { Electrical burn } \\
\text { injury }\end{array}$ & 29 months & Right & Left \\
\hline 2 & Male & 29 & $\begin{array}{c}\text { Traumatic } \\
\text { degloving injury }\end{array}$ & 20 months & Right & Left \\
\hline 3 & Female & 26 & $\begin{array}{c}\text { Tumor and } \\
\text { compartment } \\
\text { syndrome }\end{array}$ & $\begin{array}{c}60 \text { months } \\
(5 \text { years })\end{array}$ & Right & Right \\
\hline
\end{tabular}

Table V - British Medical Research Council power assessment of the affected limb after brachial plexus reconstruction and before amputation. The values above the elbow did not change after amputation

\begin{tabular}{|c|c|c:c:c|}
\cline { 3 - 5 } \multicolumn{2}{c|}{} & Patient 1 & Patient 2 & Patient 3 \\
\hline \multirow{2}{*}{ Shoulder } & Abduction & M5 & M5 & M5 \\
\cline { 2 - 5 } & Adduction & M5 & M5 & M5 \\
\hline \multirow{2}{*}{ Elbow } & Flexion & M5 & M5 & M4 \\
\cline { 2 - 5 } & Extension & M5 & M5 & M4 \\
\hline \multirow{2}{*}{ Wrist } & Flexion & M1 & M4 & M1 \\
\cline { 2 - 5 } & Extension & M1 & M4 & M1 \\
\hline \multirow{2}{*}{ Finger } & Flexion & M1 & M2 & M1 \\
\cline { 2 - 5 } & Extension & M1 & M1 & M1 \\
\hline
\end{tabular}

Table VI - Active range of motion for each patient of the affected limb after injury but before amputation. The values above the elbow did not change after amputation. All shoulder range of motions were unaffected.

\begin{tabular}{|c|c|cc|cc|cc|}
\cline { 3 - 7 } \multicolumn{2}{c|}{} & \multicolumn{2}{c|}{ Patient 1 } & Patient 2 & \multicolumn{2}{l|}{ Patient 3 } \\
\hline Elbow & Extension/Flexion & 0 & 135 & 30 & 90 & 85 & 100 \\
& Extension/Flexion MCP II-V & 0 & 5 & 0 & 0 & 0 & 0 \\
& Extension/Flexion PIP II-V & 0 & 0 & 0 & 0 & 0 & 0 \\
& Extension/Flexion DIP II-V & 0 & 0 & 0 & 0 & 0 & 0 \\
& Extension/Flexion Wrist & 5 & 5 & 10 & 5 & 0 & 0 \\
& CMC Thumb & 0 & 0 & 5 & 5 & 0 & 0 \\
& Extension/Flexion MCP I & 0 & 0 & 0 & 0 & 0 & 0 \\
& Extension/Flexion IP I & 0 & 0 & 0 & 0 & 0 & 0 \\
& Intrinsic Muscle Function & 0 & 0 & 0 & 0 & 0 & 0 \\
\hline
\end{tabular}


Patient 1 (male) sustained an electrocution injury in February 2008 at the age of 21. Both hands were severely injured requiring admission to the Intensive Care Unit for several weeks. Both arms were acutely fasciotomized and multiple surgeries followed to salvage the extremities. On his dominant right hand, the thumb and parts of the middle finger had to be amputated. On the left forearm the entire volar compartment had to be removed and the fifth finger amputated. The soft tissue defect was acutely reconstructed with a large groin flap. After consolidation of all wounds, the right thumb was reconstructed with single homologous finger transplantation, using the fourth finger of his left hand. Six months later, a myocutaneous free flap from his left thigh was transplanted with vascularized fascia lata strips in an attempt to reconstruct finger flexion of his left hand. Additionally, a long vascularized ulnar nerve graft was used to reconstruct median nerve function. Even though the surgery was successful, the trophic defects of the hand itself were so great that the hand was intrinsic minus and insensate (Figure 12 (left), Table V and Table VI). However, EMG signals were recordable from the median nerve activity in the forearm, and the remaining muscles of the extensor compartment could serve as radial nerve signals. As such, after careful assessment and discussion with the patient, an elective transradial amputation was performed to allow prosthetic replacement.

Patient 2 (male), an industrial worker, suffered a degloving injury of his entire left arm and an avulsion of his right adductor pollicis muscle in February 2012 at the age of 28. After multiple debridements (including muscles, tendons, nerves and both ulnar and radial artery at the forearm), split skin grafts, and negative pressure dressing, over the course of many weeks, the hand was buried under the abdominal skin for soft tissue recovery. Despite these reconstructive efforts the fingers had to be finally amputated at the proximal interphalangeal joints. The patient was discharged from hospital after two months of inpatient treatment. There was still hope of regaining some degree of hand function, but the remaining joints were stiff (Table $V$ and Table $\mathrm{VI}$ ) and the skin quality continually degraded to open wounds. The hand was completely insensate. In addition, at the elbow and the amputation stumps, the poor skin quality led to recurrent ulcerations and bone protrusions. Unfortunately, attempts at using orthotic supports to encourage remaining hand movement failed due to further skin breakdown (Figure 12 (centre)). After careful assessment and discussion with the patient and prosthetist, the best level of amputation was determined to save remaining forearm and wrist function for optimal prosthetic use. Thus, an elective transmetacarpal amputation was performed. Additionally, the chronic ulceration over the left olecranon was covered with a free vascularized latissimus dorsi flap together with an arthrolysis of wrist and elbow to enhance range of motion of these joints.

Patient 3 (female) fractured both ulna and radial bones of her right forearm accidentally in July 2007 in Mexico at the age of 20. In a follow up appointment later that year by the local surgical team, formation of tumors in the upper forearm were 
noted, with concerns that these might be related to rejection of implanted plates and screws. During the operation to remove these implants, tumor formation was additionally observed in the surrounding muscle, and was later diagnosed as a benign, but aggressive, desmoid tumor. Three operations followed due to residual tumor formation, together with perfusion chemotherapy. The chemotherapy provoked an arterial spasm leading to the development of compartment syndrome of both the volar and dorsal right forearm. In February 2012, an attempt to restore some hand function with z-plasties and tendon transfers was not successful. A further fracture of both radius and ulna occurred during aggressive attempts of intraoperative mobilization of the elbow, and was subsequently treated conservatively with a whole arm cast. Due to delayed bone healing the cast was in situ for 8 months. After removal, the elbow was stiff and the patient had developed a "frozen hand". In June 2013 the patient was referred to the surgical authors of this paper. The hand was completely atrophied with no useful sensation (Table V and Table VI), but she still had some activity in her flexor and extensor muscles in the upper forearm, which had no functional effect on the hand or wrist (Figure 12 (right)). The patient had previously been offered an above elbow transplantation by a separate surgical team, but declined in favour of bionic reconstruction with the main motivation of being of child bearing age without completed family planning. After assessment, discussion, intensive EMG signal training and hybrid fitting, an arthrolysis and tendon lengthening at the elbow was performed to allow adequate range of motion. An elective transradial amputation was then done to allow prosthetic replacement.

In all of these patients (Table IV) previous biological reconstruction had been attempted, as described above, but resulted in a stiff, insensate, and intrinsic minus hand, together with insufficient skin and soft tissue coverage. These characteristics were the indications to undergo bionic reconstruction. The absolute exclusion criteria were the presence of useful sensation in the hand, or severe damage to the elbow and shoulder, which would result in an inability to lift the forearm against resistance $<$ British Medical Research Council Power 4) or stabilize the shoulder joint.

\subsubsection{Clinical Evaluation}

All participants have undergone the initial screening in the specialist hand clinic. Neurological and musculoskeletal examination of upper limb function was performed including high-resolution ultrasound and nerve conduction studies to assess the state of existing muscles and nerves. In addition, quality of life metrics (Short-Form 36) and related pain scores [139] were evaluated. Thorough psychological evaluation of all patients was performed by a qualified psychologist both before and after the intervention. The evaluation contained a structured clinical interview (SCID), Freiburger assessment to evaluate adequate coping strategies (FKV-LIS) and behavioral observations [151]. 


\subsubsection{Tech-Neuro-Rehabilitation Program}

Cognitive training was started straight after the surgical reconstruction took place (Figure 13A) and before the amputation. Surface EMG was used to provide visual feedback to patients in order to train and ensure strong, consistent muscle electrical activity. This biofeedback was provided on a computer screen, in form of graphs that responded accordingly to the activation of each individual electrode. Once satisfactory signals were obtained, patients were presented with a virtual hand which then simulated the control performance of a desired prosthetic device (Figure 13B). While the non-functioning hand was in place, the use of virtual rehabilitation encouraged the patients by demonstrating that they still could control hand function. Repeated surface EMG recordings showed that this training improved signal quality and control. The patients could then practice the different functions of the prosthesis through virtual rehabilitation before the actual fitting. The virtual hand reproduced the functions and physical constraints of the real prosthesis. This process took between a few days and several weeks depending on the cognitive training requirements of the patient and the time elapsed since injury. Once confident in the virtual environment, the patients were fitted with a "hybrid hand", where a prosthetic hand was attached to a splint-like device fixed to their remaining hand (Figure 13B). The device provided direct proof for the patients that they could achieve better hand function using the prosthesis than their non-functioning biological hand. As it is expected in standard myoelectric prosthesis rehabilitation, the patients needed a few intensive hours of training to become familiar with prosthetic function. Depending on their outcome with the virtual and hybrid prosthetic systems, the patients were offered different control algorithms and were tested with them. The control algorithms evaluated by the patients were either based on direct control of 1-2 DoF or on a more advanced processing to extract the maximum number of control signals. The advanced processing applied in this study has been adapted from the method described in Amsuess et al. [52] and allows the concurrent and proportional activation of rotation, flexion, and extension of the wrist and two fine proportional grasping types [67]. This control type therefore allows an increase of the available DoFs from 1-2, typical of direct control, to 3.5, allowing a higher degree of function and versatility. The advanced control tested is natural and intuitive, without the need to switch between DoFs or between simultaneous activation of DoFs and single DoF control [67]. Testing different possibilities of control

guided further rehabilitation treatment and supported the patient's decision making process. 


\subsubsection{Amputation in Preparation for Hand Prosthesis Fitting}

After the tech-neuro-rehabilitation program had been performed successfully, the amputation of the non-functioning hand was planned in order to fit the prosthetic limb (Figure 13C). According to the anatomical status of the patient and to the requirements of the fitting prosthesis, the adequate distance for amputation varied on the individual patient's remaining functional anatomy. Patient 2 had useful remaining wrist movement, so a transmetacarpal amputation and fitting with customized short hand prosthesis was performed. Depending on the remaining sensation of the forearm, the most sensitive skin surface was used for coverage to obtain a fully sensate stump for better prosthetic fitting and feedback. Early after amputation a compressive garment was applied for edema control. Prosthetic training and fitting then could be applied as early as six weeks post amputation (Figure 13D).

\subsubsection{Materials}

Patient 1, 2 and 3 were respectively fitted with commercially available prosthetic hands: Michelangelo hand, Transcarpal Hand, and Myobock Hand - all by Otto Bock Healthcare Products $\mathrm{GmbH}$, Vienna, Austria. All patients were trained with direct proportional control, for which all electronics were embedded in the prosthesis, enabling them to take their prosthesis home and use on a daily basis.

In addition, for advanced control purposes, the Michelangelo hand for Patient 1 was equipped with a prototype wrist flexion/extension (not present in the commercial version of the prosthesis) and rotation unit. This setup, including the lateral and pinch grasp provided by the hand, allowed manipulation of 3.5 DoFs. The whole prosthesis was mounted on a custom fit socket containing eight Otto Bock raw signal electrodes (type $13 \mathrm{E} 200=50 \mathrm{AC}$ ). The socket itself housed the power unit as well as the AxonBus system, which allowed EMG sampling at the rate of $1000 \mathrm{~Hz}$ with 10 bit resolution. Electronics embedded into the electrodes provided the amplification and initial filtering of the signals. For advanced control, Bluetooth connection was established between the AxonBus and a PC, allowing the main computational burden and patient training to be transferred to a more convenient hardware.

\subsubsection{Control Algorithms}

After the training phase, it was decided that patients 2 and 3 should use conventional direct control. They could articulate at least one single DoF and the speed of the prosthetic was linearly correlated to the level of activity of the dedicated agonist or antagonist muscle depending on the direction within the DoF. Patient 3 was fitted with the rotation unit, in addition to hand opening/closing, and therefore was given an opportunity to switch between DoFs using co-contraction of agonist/antagonist with classic control. Moreover, Patient 1 presented sufficient signal quality to attempt advanced simultaneous and proportional control of the multifunctional prosthesis, 
with the possibility of acting intuitively on 3.5 DoFs [67] (Appendix II). The tests on this patient are reported for both classic control algorithms as well as for the more advanced algorithms. The classic control algorithms were those for which the patient was first trained and which he used at home.

\subsubsection{Software Framework}

The electrodes and signal conditioning were integrated into the sockets of the prostheses, which also included batteries and therefore were stand-alone systems.

For the sake of convenience and the empowerment of the advanced control system, communication between the prosthesis and a PC was established via Bluetooth. A custom developed software framework was used to handle this communication allowing acquisition and processing of the data, decision-making, prosthesis control, as well as patient training in real time.

In order to enhance the efficacy of the patient training, a visualization tool provided a polar chart of the root mean square (RMS) EMG values as a function of the electrode position. In this way a structured training program was made available, promoting the user's familiarization with the control scheme.

In order to train the system with the representative data set, the framework was granted a function to collect EMG activations in the standard pattern recognition manner. Initially, it prompted the user to elicit maximal long-term voluntary contractions (MLVC) of each controllable motion for calibration. Next, it imposed a set of trapezoidal cues with plateaus reaching $30 \%, 60 \%$ and $90 \%$ of the calibrated MLVC for movement. In this way the participant was prompted to slowly step in, hold and step out of the desired motion while constantly receiving feedback of the current contraction level based on the cumulative RMS value across all eight electrodes. Each cue was treated as a single trial lasting in total $5 \mathrm{~s}$ with the trapezoid's plateau of $3 \mathrm{~s}$. Considering the three desired levels of activation, seven of the prosthetic functions and no movement as a separate class summed up to a total of 24 trials forming a single run. Three runs recorded in relaxed, reaching in front and reaching across arm positions, were used for training the system.

\subsubsection{Functional Assessment}

Regained hand function was evaluated using a battery of established clinical tests including those that resemble activities of daily living, both using the hybrid system with the prosthesis mounted in parallel to the non-functioning hand and after elective amputation and prosthetic fitting. The global upper extremity function was evaluated both pre- and post-intervention with the Action Research Arm Test (ARAT), Southampton Hand Assessment Procedure (SHAP), and the Disabilities of the Arm, Shoulder and Hand (DASH) questionnaire, which monitor hand and upper extremity function closely related to activities of daily living [78], [137], [138]. All tests were 
performed in accordance to the standardized protocols. The DASH was not performed using the hybrid device, as it was a training device not available at the patients' homes (a requirement of the DASH questionnaire).

\subsubsection{Pain and Quality of Life Assessment}

Pain scores were evaluated using visual analogue scales (VAS). SF-36 Health Survey (German Version, 4-week recall) [139] was used to quantify the changes of quality of life after bionic reconstruction in all study patients. The questionnaire addresses eight independent subscales: physical functioning, physical role functioning, bodily pain, general health, vitality, social role functioning, emotional role functioning, and mental health. Each listed subscale ranges from 0 to 100, where the latter represents the maximum. Based on the subscales, two superior physical and mental component summary scales can be identified. These have mean values of 50 and a standard deviation of 10. For example, a patient with a psychological sum scale of 65 exhibits above average mental health compared against published age- and sex-matched norm samples of an overall representation for the German population. All the patients completed the questionnaire both prior to bionic reconstruction and after the prosthetic device has been incorporated into their regular daily living. 


\section{Neural decoding for improved force estimation in TMR patients}

\section{This chapter has been based on the work currently under revision:}

Farina, D., Vujaklija, I., Kapelner, T., Sartori, M., Negro, F., Jiang, N., et al. Man-machine interfacing with discharge timings of spinal-motor neurons after targeted muscle reinnervation. Nature Biomedical Engineering

Modern neurorehabilitation technologies are aiming to deliver natural and intuitive control. In order to achieve it, an interface based on biological signals which explores all the properties of neural drive is needed [136], [152]. In this way, a gateway for assessing the information on user's intent can be established and used for controlling a prosthetic device. This can be done at various levels of neuromuscular system - brain, nerve or muscle [67], [81], [153]-[156]. Though, the most reliable level of interaction is considered to be at the most distal of three - muscle [81], [108].

However, recordings made at the muscular level are only possible when the remaining tissue following the amputation is available and functional to a sufficient extent. Therefore, in cases of very proximal amputations up until recently very limited number of control signals could have been retrieved. With the development of TMR an opportunity for reinnervating the muscles in the proximity of the amputation was made possible, using the nerves that once carried the neural code to the affected limb [12], [14], [16], [17].

Following the TMR, an insight into efferent activity of any nerve can be made using EMG recordings. This, in principle, allows indirect detection of the neural drive descending from the spinal cord which is dispatched by motor neurons. However, current TMR based man-machine interfaces do not exploit this fact fully, but rather describe the elicited activation through a few global features [157]. In addition, by operating in the quite rigid space of designated movements [158] not much is left for the development of flexible control paradigms.

In this study a preliminary investigation into a possible more accurate neural interface is made. A proportional control signal is extracted from the decoded discharge timings of motor neurons. These spike trains have been retrieved through EMG deconvolution in three transhumeral patients who have undergone TMR. In this way a man-machine interface with an accurate physical output has been established based on information that has been retrieved from the outputs of the circuits in the spinal cord. In addition this was done in a clinically viable way through a combination of surgical procedures and advanced neural decoding. Preliminary results presented here indicate the superiority of this approach to the current state-of-the-art (SoA) in terms of ability to modulate the reconstructed force estimate. 


\subsection{Results}

Three transhumeral patients (Table VII) who have undergone TMR surgery participated in an experiment aimed to assess the use of motor neuron spike trains for proportional control. The purpose was to see whether this kind of approach could have implications on direct control methods following TMR. The 64 channel EMG recordings were successfully decomposed using CKC algorithm [103] and identifying on average $11.8 \pm$ 3.8 motor units across all three patients. An example of linearly increasing and decreasing intensity of activation by patient T1 (Table VII) is shown in Figure 15. By decomposing the generated EMG during linear increase and decrease of the activation level, two underlying mechanisms have been qualitatively revealed: recruitment of additional motor neurons and modulation of the discharge frequency of the active motor neurons (Figure 15C). In addition, high correlation has been found between the information on the population activity of motor neurons and the intensity of muscle activity. This was repeatable over different trials of the same task (Figure 15).

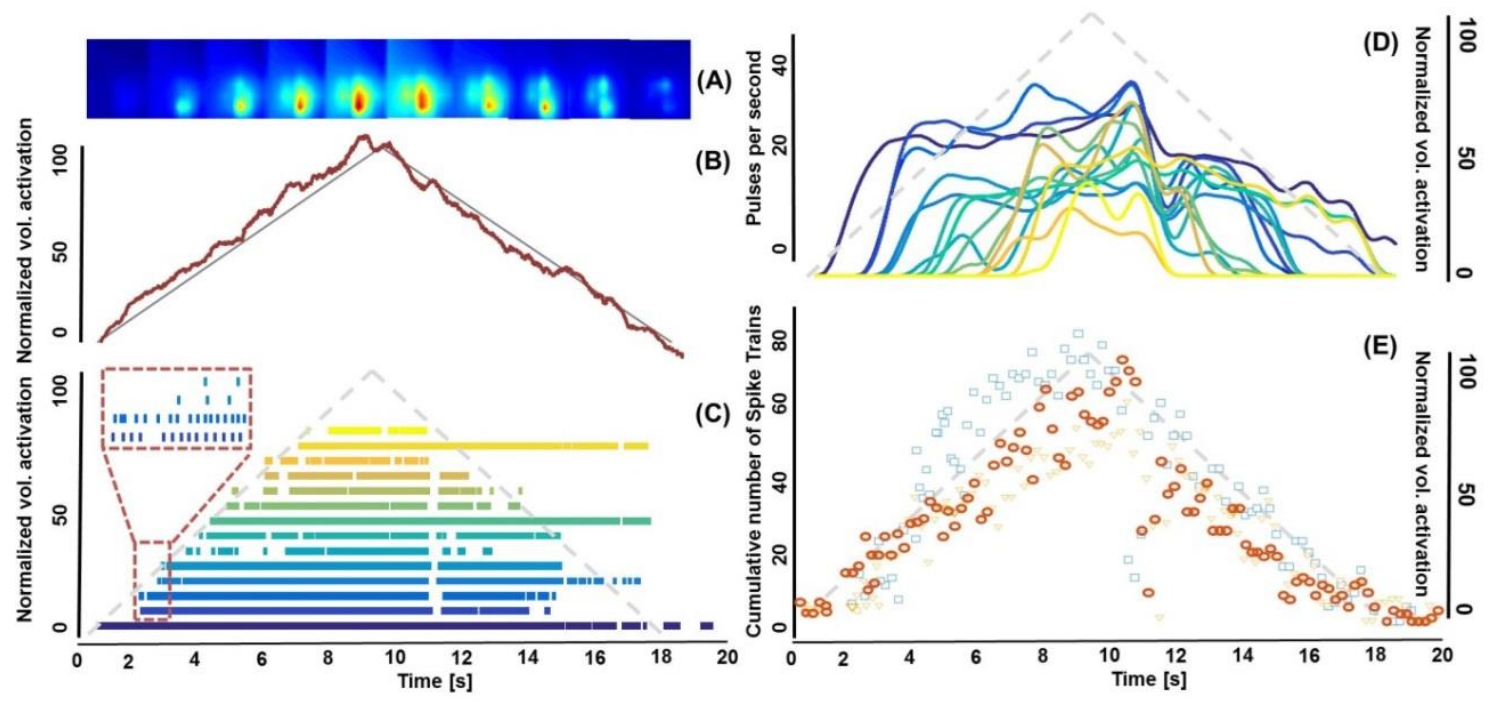

Figure 15 - Motor neuron behaviour ramp like activation in patient T1. (A) Amplitude maps of the interference EMG during a contraction of increasing and decreasing force (linear). (B) Prompt that was given to the patient as visual feedback to modulate the intensity of the contraction is shown as a black line. The surface EMG amplitude is shown as a red line. (C) Spike trains of motor neurons decoded through EMG decomposition. (D) Smoothed discharge rates of individual motor neurons with respect to the cue. (E) Instantaneous discharge rate estimated over intervals of $\mathbf{2 0 0} \mathrm{ms}$ computed from the cumulative spike train of the decoded motor neurons for three repetitions of the same task (circles; each repetition is represented with a different color), showing the association between the instantaneous rate of motor neurons and intensity of activity. The behavior of motor neurons during the ramp contractions in this representative example fully reflects the general observation on all patients tested and all trials. This is evident from the results on each patient shown in Figure 4 (lower panel).

Observations made for patient T1 were confirmed in the other patients from the same pool. Contraction intensities for one patient are presented in Figure 16. The variability of the estimates for all three patients across different processing windows can be seen in the lower panels of Figure 16. 

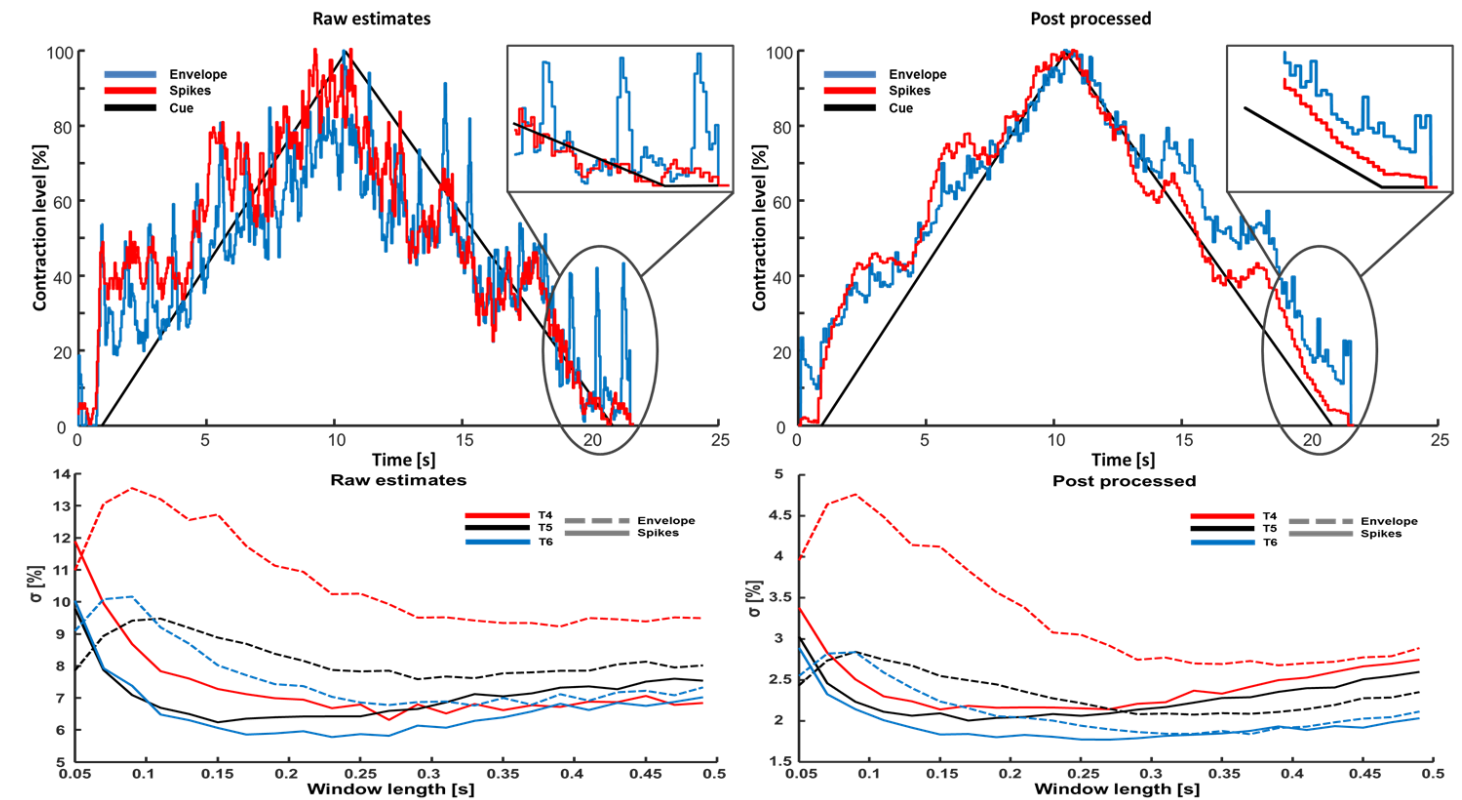

Figure 16 - Force estimates without (left column) and with (right column) post processing based on averaging over three consecutive past intervals. The plots in the top panels (representatively for patient T4) show the comparison between force estimates based on the EMG envelope (blue) and motor neuron spike trains (red) with respect to the cue (black). The bottom panels show the standard deviations $(\sigma)$ of estimation (colour coded for each of the three patients T4-T6) after linear de-trending when varying the processing interval, without (left) and with (right) post processing.

\subsection{Discussion}

This study presented a concept of a new neural interface for proportional control of neurorehabilitation technologies based on the discharge timings of spinal motor neurons. Proposed gateway to human neural day was achieved by a combination of TMR, which connects the axons of the target motor neurons to available muscle fibres, and the decoupling through deconvolution of the electrical activity of the muscle fibres innervated by each axon obtained from multi-channel EMG recordings. It has been shown that this kind of interface allows generation of meaningful and accurate control signal which can be used for novel prosthetic control. Presented technique outperformed standard interface based on classic EMG.

Using the proposed interface, a large number of motor neurons was detected from the observed reinnervation site in each of the patients. Clear assessment of the decoded neural information was possible due to accurate identification of these motor neurons. This allowed the proposed interface to go beyond the usual global description of EMG based on either amplitude or frequency features. The obtained result can be compared to the decoding of the output circuits of the spinal cord and to the identification of the spike trains directly from axonal interfacing with implanted electrodes. Though here, differently from the implanted nerve recordings, observation of the greater number of motor neurons was made. In addition, identification of the complete series of discharges was possible for the decoded motor neurons, while during the direct nerve interfacing error rate is close to $30 \%$ with 5 to 6 decoded MUs on average. This leads 
to a conclusion that using the proposed interface, motor neuron spike trains can be effectively used as relevant source of neural information for generating prosthetic commands.

The accuracy of the proposed interface has been proven through a fairly standard scenario of single DoF proportional control. The decoded neural information allowed a finer separation of intensity levels while the classic surface EMG amplitude based approach has proven itself to be more coarse. Theoretically speaking, the extracted neural information has a potential to exactly predict force [159], while on the other hand surface EMG relays on the signal variance which is constrained by the bandwidth of the signal [160].

\subsection{Methods}

\subsubsection{Patients}

Total of three patients (Table VII) participated in this study. All of them are traumatic transhumeral amputees who have undergone TMR surgery at the Medical University of Vienna. During the procedure medianus, ulnaris, and radialis nerves have been transferred into the brachialis, caput breve bicipitis, and caput laterale tricipitis muscles, respectively. This study has been approved by the local ethics committee "Ethikkommission der Medizinischen Universität Wien", approval number 1234/2015. All participants have read and signed informed consents before experiments were conducted.

Table VII - Transhumeral TMR Patinets' characteristics

\begin{tabular}{|c|c|c|c|c|c|c|c|c|}
\hline \multirow{2}{*}{ Pat. } & \multirow{2}{*}{ Age } & \multirow{2}{*}{ Sex } & Amputation & \multicolumn{2}{|c|}{ Time since } & \multicolumn{2}{|c|}{$\begin{array}{c}\text { TMR site in } \mathrm{m} . \\
\text { Bicpesc brachii }\end{array}$} & $\begin{array}{c}\text { TMR site } \\
\text { in } \mathrm{m} . \\
\text { Caput } \\
\text { laterale } \\
\text { tricipitis }\end{array}$ \\
\hline \hline T1 & 31 & $\mathrm{M}$ & $\begin{array}{c}\text { Transhumeral, } \\
\text { left }\end{array}$ & $2 \mathrm{y}, 3 \mathrm{~m}$ & $9 \mathrm{~m}$ & radialis & ulnaris & radialis \\
\hline T2 & 17 & $\mathrm{M}$ & $\begin{array}{c}\text { Transhumeral, } \\
\text { left }\end{array}$ & $>5 \mathrm{y}$ & $4 \mathrm{y}, 2 \mathrm{~m}$ & radialis & ulnaris & radialis \\
longum & $\begin{array}{c}\text { Caput } \\
\text { breve }\end{array}$ & radialis \\
T3 & 51 & $\mathrm{M}$ & $\begin{array}{c}\text { Transhumeral, } \\
\text { left }\end{array}$ & $>10 \mathrm{y}$ & $4 \mathrm{y}, 4 \mathrm{~m}$ & radialis & ulnaris & radialis \\
\hline
\end{tabular}




\subsubsection{Experimental set-up and protocol}

For the purpose of this study an electrode grid containing 64 sensors has been placed over the reinnervated short head of the biceps using double adhesive foam. Signals sensed in this way were further acquired using a multichannel biosignal amplifier (OTBioelettronica EMGUSB2). Gain was set at either 500 or 1000 and the sampling rate used was $2048 \mathrm{~Hz}$ with the sampling depth of $12 \mathrm{bits}$. In hardware realized band pass filter had cut-off frequencies at 3 and $900 \mathrm{~Hz}$. Schematic representation of the set-up is shown in Figure 17.

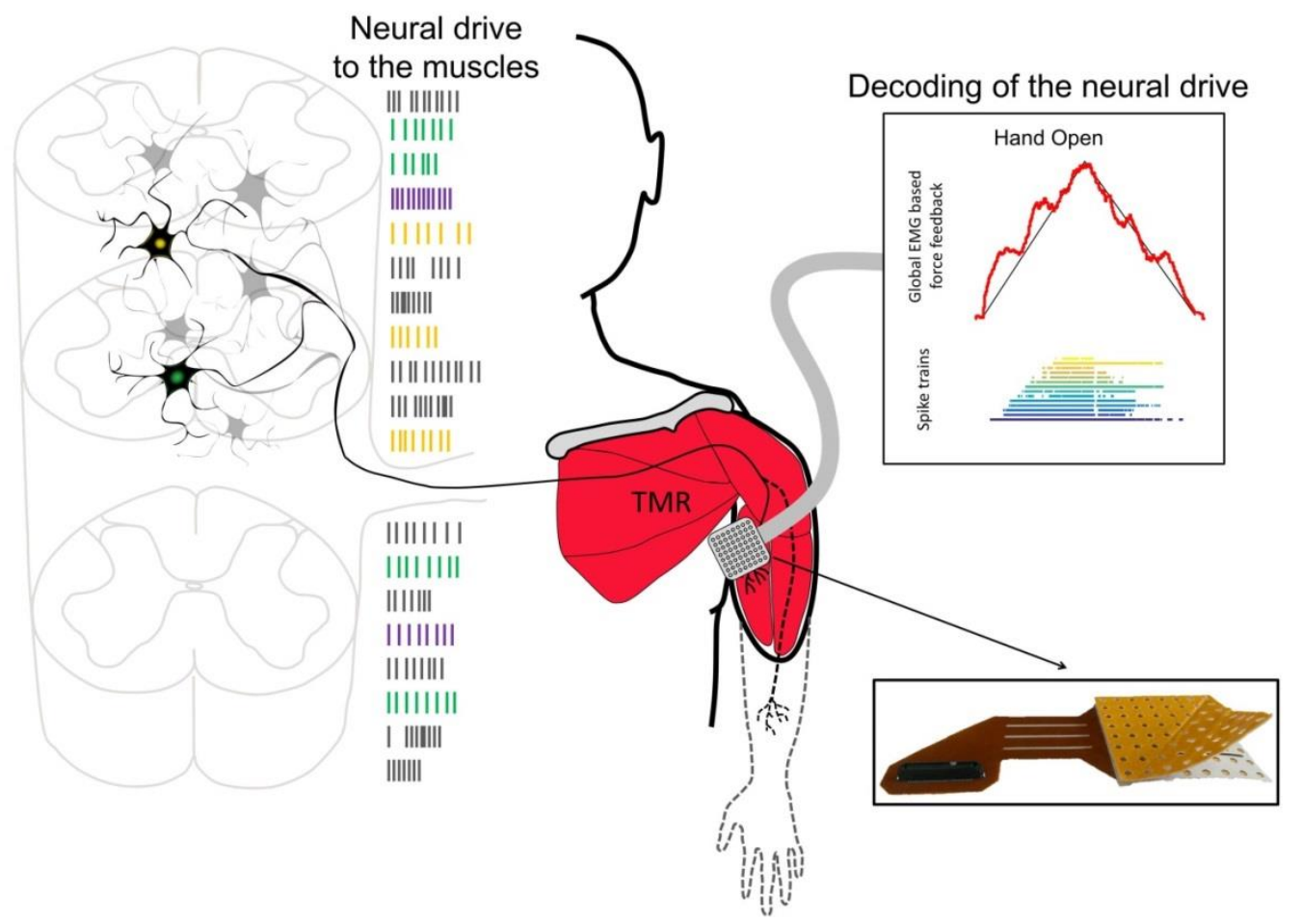

Figure 17 - Interfacing spinal motor neurons in humans. Following TMR, nerves are redirected to innervate neighbouring muscles which are treaded as biological amplifiers of nerve activity. The spike trains discharged by the innervating motor neurons are decoded by deconvolution of the surface EMG signals. The spike trains are then used to generate the proportional control signal for potential neurorehabilitation applications. The spike trains shown here are extracted from data acquired from patient T1. These spike trains are represented with the sole purpose of describing the general concept proposed in this work.

The patients seated comfortably facing a computer screen. One surface EMG electrode grid was mounted over the reinnervated short head of the biceps. Initially, all participants were asked to perform a maximal voluntary contraction by attempting a hand open gesture of their missing limb. The maximum EMG envelope across all EMG channels during this task was taken as reference for providing feedback in percent of the maximum intensity. Each subject was then prompted to increase and decrease the intensity of muscle activity from the relaxation state to the maximal intensity, over 20 s. This was done using a visual cue in a form of a ramp, which was then supposed to be matched using a cursor which changed its vertical position in relation to the muscle activation. 
The intensity of muscle activation was estimated using the EMG envelope, as a classic reference approach, and the spike trains of the identified motor neurons. The EMG signals were decomposed by a blind source separation algorithm [161] in order to provide the discharges of activation of the innervating motor neurons. These approaches were compared varying the processing interval from $50 \mathrm{~ms}$ to $500 \mathrm{~ms}$, with an interval overlap of $50 \%$. Moreover, a post processing was applied by averaging over three consecutive past intervals. For each condition, the standard deviation of the intensity estimate, after linear de-trending, was computed to determine the accuracy in the control. 


\section{Thesis discussion and conclusions}

The work presented in this thesis addressed the problem of deriving high quality neural information in order to provide a basis for a superior control of neurorehabilitation systems to users with upper limb deficiencies. Furthermore, this approach has been implemented and tested on some of the most difficult patient pools for which very limited solutions were previously available. The strong translational efforts are made throughout the studies, resulting in successful clinical implementations of the majority of the developed approaches.

The current market of upper limb prosthetics offers a variety of highly dexterous devices, though a major complain is directed towards the way in which they are controlled. Conventional cable driven systems offer limited range of motion and allow steering of just a few degrees of freedom (DoFs) which are usually coupled. Furthermore, they rely on presence of specific anatomy and patient stature while delivering somewhat unnatural control. Alternative myoelectric systems overcome some of these issues by translating the amplitude of the sensed electrical activity of the muscle contractions into the velocity of the currently controlled DoF. Through a simple state machine approach, relying on simple tricks, users of these systems are able to access a variety of DoFs and to control them in a proportional way. However, it is clear that this is not the most intuitive way of interacting with the world and cycling through individual DoFs can be rather frustrating and cumbersome.

Academia has been addressing these issues in the past decades in various ways, out of which those based on machine learning approaches seemed to be the most promising ones. Though, their commercialization hasn't quite taken off, mostly due to robustness issues and still not fully intuitive control. These approaches base themselves on a fact that the electromyographic (EMG) signals originating from various motions are essentially different once properly described. However, all the descriptors used so far are based on amplitude or spectral moments of the observed signals.

In addition, both approaches are unable to provide functional improvements in critical clinical cases. For instance, in case of high level amputations, the number of DoFs which needs to be controlled is large yet muscles, which are commonly used as sources of control signals, are few. This leaves classic machine learning based solutions with very few inputs to work with resulting in poor performance. Industrial one-to-one systems have been able to prosper from surgical interventions such as TMR, though the delivered control is still quite rudimental and overwhelmingly crude. On the other 
hand, in patients who have suffered critical soft tissue injuries it is hard to gain access to the usually intact neural drive do the remaining anatomy which now represents more of the impediment than anything else. In these cases, both academic and industrial solutions are currently standing little to no chance of delivering any functional benefit.

Even though research into neurorehabilitation technologies has yielded many interesting concepts, the way academia classifies which of them might be promising in everyday life seems not to be delivering quite as expected. As the first study of this thesis, concept of offline testing of myoelectric systems has been re-evaluated. Most commonly used classification accuracy score has been compared to clinically recognized tests. Correlation between them was shown to be poor indicating necessity for improving or further shifting the academic evaluation tools towards clinically used ones. Furthermore, during this process, the validity of clinical tests themselves has been questioned. The work presented here indicates that even these scores fail to account for all major parameters describing ones functionality given the tested technology. Main issue arises from the fact that the majority of clinical evaluations rely strictly on whether or not the tested subject is able to perform the given task and in which time. What is neglected is the quantitative description of the way that the task has been done. Here, it is shown through a side by side comparison of an industrial and an academic flagship, transradial solutions that even though the former one has obtained slightly better clinical scores, the amount of compensatory movements expressed in the later is smaller. This leads to a conclusion that the system proposed by academia is offering more natural control which on a long run might provide higher quality of life to a prosthetic user. Moreover, the significance of translational activities within myocontrol research has been strongly outlined in this study and it has been shown that despite all the challenges clinical implementation of the newly proposed systems is of out most importance.

As aforementioned, the majority of patients with critical soft tissue upper limb injury currently cannot be provided with any of the available neurorehabilitation technologies in order to sufficiently increase or substitute their lack of functionality. Case series presented in this thesis lays down a viable solution to this problem. A combination of surgical techniques and engineering solutions was developed as an extension of bionic reconstruction technique previously implemented for improving everyday experience in patients suffering from brachial plexus injuries. This technique, through means of surgical interventions, elective amputation, tech-neurorehabilitation and advanced human-machine interfacing enabled access and utilization of the neural drive present in patient suffering from this specific, yet not so rare, disability. As shown here through a set of clinical tests conducted before, during and post intervention the functional improvement is high and rewarding. Through a battery of psychological tests and testimonials by all of the tested patients, success of 
this approach extends beyond functionality. Namely, all three patients in this case series managed to reintegrate into the society and continue their lives with a higher level of social and emotional engagement. This study was yet another example in which strong translational approach to a very complex problem led to a clinical and finally real world application of a laboratory based concept.

Finally a concept of using high level neural information, which was made accessible by TMR in transhumeral amputees, for potentially delivering precise proportional control signal was investigated. Namely, by deconvolution of the HD EMG signals obtained from reinnervated muscles the MU spike trains were obtained allowing precise and highly modulated reconstruction of the intended force signal. On a small pool of three patients it has been shown that this approach is able to potentially deliver finer and more articulated output which could be of use for driving various neurorehabilitation technologies. This might be the study which is at this point furthest from a clinical scenario, but on a long run, introduction of this indirect interface to spinal cord, into already available TMR prosthetic solutions could deliver more precise and natural steering over all driveable DoFs. Therefore, this result is putting a strong basis for future clinically viable solutions which could revolutionize the way novel neuroethologies are being interfaced.

The outcomes of this thesis strongly support the idea that the engineering research in man-machine interfacing for prosthesis control needs to be evaluated in real clinical cases relying on extensive clinical testing. The laboratory tests are evidently not sufficient and the simplified conditions which they provide, relaying on classic metrics, are misleading and useless. The current state of the art in commercial myocontrol systems can be advanced by applying algorithms which support simultaneous and proportional control, though only if they have been extensively clinically tested. This seems to be the only way to make a sound prediction on whether or not a system could stand a chance in the real world.

In order to set a new frontier in myocontrol system design the necessity to further deepen the information extracted from muscle electrical activity seems eminent. The way to achieve this is by estimating the neural drive sent to the muscles directly, which has been proven feasible for the first time in clinically challenging conditions of transhumeral amputations.

Overall, the thesis provides new horizons for the clinical translation of advanced myocontrol. This has been achieved after decades in which the clinical transferability of new methods for man-machine interfacing, in upper limb prostheses, has been close to none. The key to this success has been in combining new surgical interventions, advanced methods for EMG detection, integrative engineering, and clinical tests. This multidisciplinary effort was only made possible through collaborations with surgeons, clinicians, physiotherapists, orthopaedics, mathematicians, and engineers. 


\section{References}

[1] R. Niska, F. Bhuiya, and J. Xu, "National Hospital Ambulatory Medical Care Survey: 2007 emergency department summary.," Natl. Health Stat. Report., no. 26, pp. 1-31, 2010.

[2] D. Ootes, K. T. Lambers, and D. C. Ring, "The epidemiology of upper extremity injuries presenting to the emergency department in the United States," Hand, vol. 7, no. 1, pp. 18-22, 2012.

[3] C. F. Larsen, S. Mulder, A. M. T. Johansen, and C. Stam, "The epidemiology of hand injuries in the Netherlands and Denmark," Eur. J. Epidemiol., vol. 19, no. 4, pp. 323-327, 2004.

[4] U.S Department of Labor and Bureau of Labor Statistics, Work related hand injuries and upper extremity amputations.pdf, Bulletin 2. Washington, D.C: U.S Department of Labor, 1982.

[5] A. P. Kahn, The encyclopedia of work-related hand injuries and upper extremity amputations, vol. 1. New York, New York, USA: Facts on File, 2004.

[6] A. C. Rettig, "Athletic Injuries of the Wrist and Hand," Am. J. Sports Med., vol. 32, no. 1, pp. 262-273, 2004.

[7] V. PUTTI, "Historical Prostheses," J. Hand Surg. J. Br. Soc. Surg. Hand, vol. 30, no. 3, pp. 310-325, Jun. 2005.

[8] D. R. Merrill, J. Lockhart, P. R. Troyk, R. F. Weir, and D. L. Hankin, "Development of an implantable myoelectric sensor for advanced prosthesis control.," Artif. Organs, vol. 35, no. 3, pp. 249-52, Mar. 2011.

[9] D. Childress, "Historical aspects of powered limb prostheses," Clin. prosthetics Orthot., 1985.

[10] E. Marquardt, "The Heidelberg pneumatic arm prosthesis," J. Bone Jt. Surgery, Br. ..., vol. 47, no. 3, p. 425, 1965.

[11] T. a Kuiken, G. a Dumanian, R. D. Lipshutz, L. a Miller, K. a Stubblefield, R. D. Lipschutz, L. a Miller, and K. a Stubblefield, "The use of targeted muscle reinnervation for improved myoelectric prosthesis control in a bilateral shoulder disarticulation amputee," Prosthet. Orthot. Int., vol. 28, no. 3, pp. 245-253, Dec. 2004. 
[12] J. Hoffer and G. Loeb, "Implantable electrical and mechanical interfaces with nerve and muscle," Ann. Biomed. Eng., vol. 8, 1980.

[13] D. Farina, H. Rehbaum, A. Holobar, I. Vujaklija, N. Jiang, C. Hofer, S. Salminger, H.-W. Van Vliet, and O. Aszmann, "Non-Invasive, Accurate Assessment of the Behavior of Representative Populations of Motor Units in Targeted Reinnervated Muscles.," IEEE Trans. Neural Syst. Rehabil. Eng., vol. 4320, no. c, Feb. 2014.

[14] M. S. Gart, J. M. Souza, and G. A. Dumanian, "Targeted Muscle Reinnervation in the Upper Extremity Amputee: A Technical Roadmap," J. Hand Surg. Am., vol. 40, no. 9, pp. 1877-1888, 2015.

[15] K. A. Stubblefield, L. A. Miller, R. D. Lipschutz, and T. A. Kuiken, "Occupational therapy protocol for amputees with targeted muscle reinnervation," J. Rehabil. Res. Dev., vol. 46, no. 4, p. 481, Jan. 2009.

[16] T. a Kuiken, L. a Miller, R. D. Lipschutz, B. a Lock, K. Stubblefield, P. D. Marasco, P. Zhou, and G. a Dumanian, "Targeted reinnervation for enhanced prosthetic arm function in a woman with a proximal amputation: a case study.," Lancet, vol. 369, no. 9559, pp. 371-80, Feb. 2007.

[17] T. a Kuiken, G. Li, B. a Lock, R. D. Lipschutz, L. a Miller, K. a Stubblefield, and K. B. Englehart, "Targeted muscle reinnervation for real-time myoelectric control of multifunction artificial arms," JAMA, vol. 301, no. 6, pp. 619-628, 2009.

[18] K. Ohnishi, R. F. Weir, and T. a Kuiken, "Neural machine interfaces for controlling multifunctional powered upper-limb prostheses.," Expert Rev. Med. Devices, vol. 4, no. 1, pp. 43-53, 2007.

[19] L. a Miller, R. D. Lipschutz, K. a Stubblefield, B. a Lock, H. Huang, T. W. Williams, R. F. Weir, and T. a Kuiken, "Control of a six degree of freedom prosthetic arm after targeted muscle reinnervation surgery.," Arch. Phys. Med. Rehabil., vol. 89, no. 11, pp. 2057-65, Nov. 2008.

[20] R. L. Lieber, "Skeletal Muscel Architecture Implications for Muscle Function and Surgical Tendon Transfer," J. Hand Ther., vol. 6, no. 2, pp. 105-113, 1993.

[21] W. P. Cooney, R. L. Linscheid, and K. N. An, "Opposition of the thumb: an anatomic and biomechanical study of tendon transfers," J. Hand Surg. Am., vol. 9, no. 6, pp. 777-786, 1984.

[22] J. W. May and L. Gordon, "Palm of hand free flap for forearm length preservation in nonreplantable forearm amputation: a case report.," J. Hand Surg. Am., vol. 5, no. 4, pp. 377-380, 1980. 
[23] B. G. Ulusal, Y.-T. Lin, A. E. Ulusal, and C.-H. Lin, "Free lateral arm flap for 1stage reconstruction of soft tissue and composite defects of the hand: A retrospective analysis of 118 cases.," Ann. Plast. Surg., vol. 58, no. 2, pp. 173-8, 2007.

[24] K. Brandt, R. K. Khouri, and J. Upton, "Free flaps as flow-through vascular conduits for simultaneous coverage and revascularization of the hand or digit.," Plastic and reconstructive surgery, vol. 98. pp. 321-327, 1996.

[25] M. Neumeister, T. Hegge, A. Amalfi, and M. Sauerbier, "The reconstruction of the mutilated hand," Semin. Plast. Surg., vol. 24, no. 1, pp. 77-102, 2010.

[26] V. P. Blair and L. T. Byars, "Toe to Finger Transplant," Ann. Surg., pp. 287-290, 1940.

[27] W. A. Morrison, B. McC. O'Brien, and A. M. MacLeod, "Ring finger transfer in reconstruction of transmetacarpal amputations," J. Hand Surg. Am., vol. 9, no. 1, pp. 4-11, 1984.

[28] G. Foucher and A. L. H. Moss, "Microvascular second toe to finger transfer: a statistical analysis of 55 transfers," Br. J. Plast. Surg., vol. 44, no. 2, pp. 87-90, 1991.

[29] T. J. Canty and E. E. Bleck, "Split-thickness skin grafting of amputation stumps," Plast. Reconstr. Surg., vol. 10, pp. 99-115, 1952.

[30] M. R. Wood, G. A. Hunter, and S. G. Millstein, "The value of stump split skin grafting following amputation for trauma in adult upper and lower limb amputees," Prosthet Orthot Int, vol. 11, no. 2, pp. 71-74, 1987.

[31] J. W. Jones, S. A. Gruber, J. H. Barker, and W. C. Breidenbach, "SUCCESSFUL HAND TRANSPLANTATION One-Year Follow-up," N. Engl. J. Med., vol. 343, no. 7, pp. 468-473, 2000.

[32] S. Salminger, A. Sturma, A. Roche, M. Ninkovic, G. Pierer, A. Chelmonski, J. Jablecki, and O. Aszmann, "Handfunktion nach Handtransplantation und prothetischer Rekonstruktion," in 38. Seminar - Surgery meets Biomedical Engineering, 2014.

[33] P. Petruzzo, M. Lanzetta, J.-M. Dubernard, L. Landin, P. Cavadas, R. Margreiter, S. Schneeberger, W. Breidenbach, C. Kaufman, J. Jablecki, F. Schuind, and C. Dumontier, "The International Registry on Hand and Composite Tissue Transplantation," Transplantation, vol. 90, no. 12, pp. 1590-1594, Dec. 2010.

[34] E. Biddiss and T. Chau, "Upper-limb prosthetics: critical factors in device abandonment.," Am. J. Phys. Med. Rehabil., vol. 86, no. 12, pp. 977-87, Dec. 2007. 
[35] C. Pylatiuk, S. Schulz, and L. Döderlein, "Results of an Internet survey of myoelectric prosthetic hand users," Prosthet. Orthot. Int., vol. 31, no. 4, pp. 362-370, 2007.

[36] M. Egermann, P. Kasten, and M. Thomsen, "Myoelectric hand prostheses in very young children," Int. Orthop., vol. 33, no. 4, pp. 1101-1105, 2009.

[37] L. V McFarland, S. L. Hubbard Winkler, A. W. Heinemann, M. Jones, and A. Esquenazi, "Unilateral upper-limb loss: satisfaction and prosthetic-device use in veterans and servicemembers from Vietnam and OIF/OEF conflicts.," J. Rehabil. Res. Dev., vol. 47, no. 4, pp. 299-316, 2010.

[38] K. Østlie, I. M. Lesjø, R. J. Franklin, B. Garfelt, O. H. Skjeldal, and P. Magnus, "Prosthesis rejection in acquired major upper-limb amputees: a population-based survey.," Disabil. Rehabil. Assist. Technol., vol. 7, no. 4, pp. 294-303, Jul. 2012.

[39] H. Silcox, M. Rooks, R. Vogel, and L. Fleming, "Myoelectric prostheses. A longterm follow-up and a study of the use of alternate prostheses.," J. Bone Joint Surg. Am., vol. 75-A, no. 12, pp. 1781-1789, 1993.

[40] S. F. Burrough and J. A. Brook, "Patterns of Acceptance and Rejection of Upper Limb Prostheses," Orthot. Prosthetics, vol. 39, no. 2, pp. 40-47, 1985.

[41] M. K. Glynn, H. R. Galway, G. Hunter, and W. F. Sauter, "Management of the upper-limb-deficient child with a powered prosthetic device.," Clin. Orthop. Relat. Res., vol. 209, no. 209, pp. 202-5, 1986.

[42] Otto Bock Healthcare GmbH, "FDA 510(k) premarket notification: Summary Axon-Bus Prosthetic System," Silver Spring, MD 20993-0002, 2014.

[43] Touch Bionics, "How the i-limb works." [Online]. Available: http://www.touchbionics.com/products/how-i-limb-works. [Accessed: 29-Apr-2016].

[44] RSL Steeper, "BeBionic v3 Product Brochure." RSL Steeper, 2013.

[45] Touch Bionics, "i-limb QR codes." [Online]. Available: http://www.touchbionics.com/resources/qr-codes. [Accessed: 29-Apr-2016].

[46] B. Hudgins, P. Parker, and R. N. Scott, "A new strategy for multifunction myoelectric control," IEEE Trans. Biomed. Eng., vol. 40, no. 1, pp. 82-94, Jan. 1993.

[47] D. Graupe, J. Salahi, and K. H. Kohn, "Multifunctional prosthesis and orthosis control via microcomputer identification of temporal pattern differences in single-site myoelectric signals.," J. Biomed. Eng., vol. 4, no. 1, pp. 17-22, Jan. 1982. 
[48] F. R. Finley and R. W. Wirta, "Myocoder-computer study of electromyographic patterns.," Arch. Phys. Med. Rehabil., vol. 48, no. 1, pp. 20-4, Jan. 1967.

[49] D. Graupe and W. K. Cline, "Functional separation of EMG signals via ARMA identification methods for prosthesis control purposes," IEEE Transactions on Systems, Man and Cybernetics, vol. SMC-5, no. 2. pp. 252-259, 1975.

[50] P. Herberts, C. Almström, and K. Caine, "Clinical application study of multifunctional prosthetic hands.," J. Bone Joint Surg. Br., vol. 60-B, no. 4, pp. 552560, 1978.

[51] B. Peerdeman, D. Boere, H. Witteveen, R. Huis in 'tVeld, H. Hermens, S. Stramigioli, H. Rietman, P. Veltink, and S. Misra, "Myoelectric forearm prostheses: State of the art from a user-centered perspective," J. Rehabil. Res. Dev., vol. 48, no. 6, p. 719, 2011.

[52] S. Amsuess, P. Gobel, B. Graimann, and D. Farina, "A Multi-Class Proportional Myocontrol Algorithm for Upper Limb Prosthesis Control: Validation in Real-Life Scenarios on Amputees.," IEEE Trans. Neural Syst. Rehabil. Eng., vol. 4320, no. c, pp. 111, Oct. 2014.

[53] A. Ameri, E. J. Scheme, E. N. Kamavuako, K. B. Englehart, and P. a. Parker, "Realtime, simultaneous myoelectric control using force and position-based training paradigms," IEEE Trans. Biomed. Eng., vol. 61, no. 2, pp. 279-287, Sep. 2014.

[54] E. Scheme and K. Englehart, "Electromyogram pattern recognition for control of powered upper-limb prostheses: State of the art and challenges for clinical use," J. Rehabil. Res. Dev., vol. 48, no. 6, p. 643, 2011.

[55] K. Englehart, B. Hudgins, and P. a Parker, "A wavelet-based continuous classification scheme for multifunction myoelectric control.," IEEE Trans. Biomed. Eng., vol. 48, no. 3, pp. 302-11, Mar. 2001.

[56] A. Stango, F. Negro, and D. Farina, "Spatial Correlation of High Density EMG Signals Provides Features Robust to Electrode Number and Shift in Pattern Recognition for Myocontrol," IEEE Trans. Neural Syst. Rehabil. Eng., vol. 23, no. 2, pp. 189-198, 2015.

[57] J. M. Hahne, F. Biebmann, N. Jiang, H. Rehbaum, D. Farina, F. C. Meinecke, K.-R. Muller, and L. C. Parra, "Linear and Nonlinear Regression Techniques for Simultaneous and Proportional Myoelectric Control," IEEE Trans. Neural Syst. Rehabil. Eng., vol. 22, no. 2, pp. 269-279, 2014.

[58] G. H. Golub, P. C. Hansen, and D. P. O'Leary, "Tikhonov regularization and total least squares," Siam J. Matrix Anal. Appl., vol. 21, no. 1, pp. 185-194, 1999. 
[59] N. Jiang, S. Muceli, B. Graimann, and D. Farina, "Effect of arm position on the prediction of kinematics from EMG in amputees," Med. Biol. Eng. Comput., vol. 51, no. 1-2, pp. 143-151, 2013.

[60] J. L. G. Nielsen, S. Holmgaard, N. Jiang, K. B. Englehart, D. Farina, and P. a Parker, "Simultaneous and proportional force estimation for multifunction myoelectric prostheses using mirrored bilateral training.," IEEE Trans. Biomed. Eng., vol. 58, no. 3, pp. 681-8, Mar. 2011.

[61] S. Muceli and D. Farina, "Simultaneous and Proportional Estimation of HandKinematics From EMG During Mirrored Movements at MultipleDegrees-ofFreedom.," IEEE Trans. Neural Syst. Rehabil. Eng., vol. 20, no. 3, pp. 371-8, Dec. 2011.

[62] N. Jiang, K. B. Englehart, and P. a Parker, "Extracting simultaneous and proportional neural control information for multiple-DOF prostheses from the surface electromyographic signal.," IEEE Trans. Biomed. Eng., vol. 56, no. 4, pp. 1070-80, Apr. 2009.

[63] D. D. Lee and H. S. Seung, "Learning the parts of objects by non-negative matrix factorization.," Nature, vol. 401, no. 6755, pp. 788-91, Oct. 1999.

[64] N. Jiang, H. Rehbaum, I. Vujaklija, B. Graimann, and D. Farina, "Intuitive, Online, Simultaneous, and Proportional Myoelectric Control Over Two Degrees-of-Freedom in Upper Limb Amputees.," IEEE Trans. Neural Syst. Rehabil. Eng., vol. 22, no. 3, pp. 50110, May 2014.

[65] N. Jiang, I. Vujaklija, H. Rehbaum, B. Graimann, and D. Farina, "Is accurate mapping of EMG signals on kinematics needed for precise online myoelectric control?," IEEE Trans. Neural Syst. Rehabil. Eng., vol. 22, no. 3, pp. 549-58, 2014.

[66] M. Ison, I. Vujaklija, B. Whitsell, D. Farina, and P. Artemiadis, "High-Density Electromyography and Motor Skill Learning for Robust Long-Term Control of a 7-DoF Robot Arm," IEEE Trans. Neural Syst. Rehabil. Eng., vol. to appear, pp. 1-10, 2015.

[67] S. Amsuess, I. Vujaklija, P. Gobel, A. Roche, B. Graimann, O. Aszmann, and D. Farina, "Context-Dependent Upper Limb Prosthesis Control for Natural and Robust Use," IEEE Trans. Neural Syst. Rehabil. Eng., pp. 1-1, 2015.

[68] M. Ortiz-Catalan, R. Brånemark, and B. Håkansson, "BioPatRec: A modular research platform for the control of artificial limbs based on pattern recognition algorithms.," Source Code Biol. Med., vol. 8, no. 1, p. 11, Jan. 2013.

[69] M. Ison, I. Vujaklija, B. Whitsell, D. Farina, and P. Artemiadis, "Simultaneous Myoelectric Control of a Robot Arm using Muscle Synergy-Inspired Inputs from High- 
Density Electrode Grids," in IEEE International Conference on Robotics and Automation, 2015, pp. 6469-6474.

[70] A. M. Simon, L. J. Hargrove, B. a. Lock, and T. a. Kuiken, "Target Achievement Control Test: Evaluating real-time myoelectric pattern-recognition control of multifunctional upper-limb prostheses," J. Rehabil. Res. Dev., vol. 48, no. 6, p. 619, 2011.

[71] E. J. Fimbel, M. Lemay, and M. Arguin, "Speed-accuracy trade-offs in myocontrol," Hum. Mov. Sci., vol. 25, no. 2, pp. 165-180, 2006.

[72] P. M. Fitts, "The Information Capacity of the Human Motor," J. Exp. Biol., vol. 47, no. 6, pp. 381-391, 1954.

[73] J. Park, W. Bae, H. Kim, and S. Park, "EMG - force correlation considering Fitts' law," 2008 IEEE Int. Conf. Multisens. Fusion Integr. Intell. Syst., pp. 644-649, Aug. 2008.

[74] E. J. Scheme and K. B. Englehart, "Validation of a selective ensemble-based classification scheme for myoelectric control using a three-dimensional fitts' law test," IEEE Trans. Neural Syst. Rehabil. Eng., vol. 21, no. 4, pp. 616-623, 2013.

[75] W. Daly, L. Voo, T. Rosenbaum-Chou, A. Arabian, and D. Boone, "Socket Pressure and Discomfort in Upper-Limb Prostheses: A Preliminary Study," JPO J. Prosthetics Orthot., vol. 26, no. 2, pp. 99-106, 2014.

[76] A. D. Roche, I. Vujaklija, S. Amsüss, A. Sturma, P. Göbel, D. Farina, and O. C. Aszmann, "A Structured Rehabilitation Protocol for Improved Multifunctional Prosthetic Control: A Case Study.," J. Vis. Exp., no. 105, p. e52968, 2015.

[77] A. Sturma, A. D. Roche, P. Göbel, M. Herceg, N. Ge, V. Fialka-Moser, and O. Aszmann, "A surface EMG test tool to measure proportional prosthetic control," Biomed. Tech., vol. 60, no. 3, pp. 207-213, 2015.

[78] C. M. Light, P. H. Chappell, and P. J. Kyberd, "Establishing a Standardized Clinical Assessment Tool of Pathologic and Prosthetic Hand Function: Normative Data, Reliability, and Validity," Arch. Phys. Med. Rehabil., vol. 83, no. 6, pp. 776-783, 2002.

[79] L. N. Hermansson, A. G. Fisher, B. Bernspang, and A. C. Eliasson, "Assessment of capacity for myoelectric control: a new Rasch-built measure of prosthetic hand control," J. Rehabil. Med., vol. 37, no. 3, pp. 166-71, 2005.

[80] E. Davis Sears and K. C. Chung, "Validity and Responsiveness of the JebsenTaylor Hand Function Test," J. Hand Surg. Am., vol. 35, no. 1, pp. 30-37, Jan. 2010.

[81] M. A. Lebedev and M. A. L. Nicolelis, "Brain-machine interfaces: past, present and future," Trends Neurosci., vol. 29, no. 9, pp. 536-546, 2006. 
[82] C. J. Heckman and R. M. Enoka, "Motor unit.," Compr. Physiol., vol. 2, no. 4, pp. 2629-82, Oct. 2012.

[83] J. Duchateau and R. M. Enoka, "Human motor unit recordings: Origins and insight into the integrated motor system," Brain Res., vol. 1409, pp. 42-61, 2011.

[84] R. Merletti and D. Farina, Eds., Surface Electromyography: Physiology, Enigineering and applications. Wiley-IEEE Press, 2015.

[85] A. B. Jenny and J. Inukai, "Principles of motor organization of the monkey cervical spinal cord.," J. Neurosci., vol. 3, no. 3, pp. 567-575, 1983.

[86] B. Feinstein, B. Lindegård, E. Nyman, and G. Wohlfart, "Morphologic Studies Of Motor Units In Normal Human Muscles," Cells Tissues Organs, vol. 23, no. 2, pp. 127142, Jul. 1955.

[87] H. S. Milner-Brown, R. B. Stein, and R. Yemm, "The contractile properties of human motor units during voluntary isometric contractions," J. Physiol., vol. 228, pp. 285-306, 1973.

[88] C. K. Thomas, R. S. Johansson, G. Westling, and B. Bigland-Ritchie, "Twitch properties of human thenar motor units measured in response to intraneural motoraxon stimulation.," J. Neurophysiol., vol. 64, no. 4, pp. 1339-46, Oct. 1990.

[89] M. Van Cutsem, P. Feiereisen, J. Duchateau, and K. Hainaut, "Mechanical Properties and Behaviour of Motor Units in the Tibialis Anterior During Voluntary Contractions," Can. J. Appl. Physiol., vol. 22, no. 6, pp. 585-597, Nov. 1997.

[90] A. J. Harris, "Muscle Fiber and Motor Unit Behavior in the Longest Human Skeletal Muscle," J. Neurosci., vol. 25, no. 37, pp. 8528-8533, 2005.

[91] T. M. M. Vieira, I. D. Loram, S. Muceli, R. Merletti, and D. Farina, "Postural activation of the human medial gastrocnemius muscle: are the muscle units spatially localised?," J. Physiol., vol. 589, pp. 431-443, 2011.

[92] L. G. Ermilov, C. B. Mantilla, K. L. Rowley, and G. C. Sieck, "Safety factor for neuromuscular transmission at type-identified diaphragm fibers," Muscle and Nerve, vol. 35, no. 6, pp. 800-803, 2007.

[93] C. B. Mantilla, K. L. Rowley, W. Z. Zhan, M. A. Fahim, and G. C. Sieck, "Synaptic vesicle pools at diaphragm neuromuscular junctions vary with motoneuron soma, not axon terminal, inactivity," Neuroscience, vol. 146, no. 1, pp. 178-189, 2007.

[94] R. L. Ruff, "Neurophysiology of the neuromuscular junction: Overview," Ann. N. Y. Acad. Sci., vol. 998, pp. 1-10, 2003. 
[95] S. J. Wood and C. R. Slater, Safety factor at the neuromuscular junction, vol. 64, no. 4. 2001.

[96] A. J. Fuglevand, D. A. Winter, A. E. Patla, and D. Stashuk, "Detection of motor unit action potentials with surface electrodes: influence of electrode size and spacing," Biol. Cybern., vol. 67, no. 2, pp. 143-153, 1992.

[97] D. Farina, R. Merletti, and R. M. Enoka, "The extraction of neural strategies from the surface EMG," J. Appl. Physiol., vol. 96, no. 4, pp. 1486-1495, Apr. 2004.

[98] G. A. Garc??a, R. Okuno, and K. Akazawa, "Surface electrode-array electromyogram decomposition," Int. IEEE/EMBS Conf. Neural Eng. NER, vol. 2003Janua, no. August, pp. 388-391, 2003.

[99] M. Gazzoni, D. Farina, and R. Merletti, "A new method for the extraction and classification of single motor unit action potentials from surface EMG signals.," J. Neurosci. Methods, vol. 136, no. 2, pp. 165-77, Jul. 2004.

[100] B. U. Kleine, J. P. van Dijk, B. G. Lapatki, M. J. Zwarts, and D. F. Stegeman, "Using two-dimensional spatial information in decomposition of surface EMG signals," J. Electromyogr. Kinesiol., vol. 17, no. 5, pp. 535-548, 2007.

[101] E. M. Maathuis, J. Drenthen, J. P. van Dijk, G. H. Visser, and J. H. Blok, "Motor unit tracking with high-density surface EMG," J. Electromyogr. Kinesiol., vol. 18, no. 6, pp. 920-930, 2008.

[102] a Holobar and D. Zazula, "Correlation-based decomposition of surface electromyograms at low contraction forces.," Med. Biol. Eng. Comput., vol. 42, no. 4, pp. 487-95, Jul. 2004.

[103] A. Holobar and D. Zazula, "Multichannel blind source separation using convolution Kernel compensation," IEEE Trans. Signal Process., vol. 55, no. 9, pp. 4487-4496, 2007.

[104] C. J. De Luca, A. Adam, R. Wotiz, L. D. Gilmore, and S. H. Nawab, “Decomposition of surface EMG signals," J Neurophysiol, vol. 96, no. 3, pp. 1646-1657, 2006.

[105] S. H. Nawab, S. S. Chang, and C. J. De Luca, "High-yield decomposition of surface EMG signals," Clin. Neurophysiol., vol. 121, no. 10, pp. 1602-1615, 2010.

[106] O. C. Aszmann, A. D. Roche, S. Salminger, T. Paternostro-sluga, M. Herceg, A. Sturma, C. Hofer, and D. Farina, "Bionic reconstruction to restore hand function after brachial plexus injury : a case series of three patients," Lancet, vol. 6736, pp. 1-7, 2015. 
[107] W. Hill, Ø. Stavdahl, L. N. Hermansson, P. Kyberd, S. Swanson, and S. Hubbard, "Functional Outcomes in the WHO-ICF Model: Establishment of the Upper Limb Prosthetic Outcome Measures Group," J. Prosthetics Orthot., vol. 21, no. 1, pp. 115119, 2009.

[108] N. Jiang, S. Dosen, and D. Farina, "Myoelectric control of artificial limbs: is there the need for a change of focus?," IEEE Signal Process. Mag., no. September, pp. 12-15, 2012.

[109] C. Cipriani, J. L. Segil, J. A. Birdwell, and R. F. Weir, "Dexterous control of a prosthetic hand using fine-wire intramuscular electrodes in targeted extrinsic muscles," IEEE Trans. Neural Syst. Rehabil. Eng., vol. 22, no. 4, pp. 828-836, 2014.

[110] S. Dosen, C. Cipriani, M. Kostić, M. Controzzi, M. C. Carrozza, and D. B. Popović, "Cognitive vision system for control of dexterous prosthetic hands: experimental evaluation.," J. Neuroeng. Rehabil., vol. 7, p. 42, Jan. 2010.

[111] C. Nissler, N. Mouriki, and C. Castellini, "Optical Myography: Detecting Finger Movements by Looking at the Forearm," Front. Neurorobot., vol. 10, no. April, pp. 110, 2016.

[112] V. Ortenzi, S. Tarantino, C. Castellini, and C. Cipriani, "Ultrasound imaging for hand prosthesis control: A comparative study of features and classification methods," IEEE Int. Conf. Rehabil. Robot., vol. 2015-Septe, pp. 1-6, 2015.

[113] R. F. Weir, P. R. Troyk, G. DeMichele, T. Kuiken, and T. Ku, "Implantable myoelectric sensors (IMES) for upper-extremity prosthesis control- preliminary work," Annu. Int. Conf. IEEE Eng. Med. Biol. Soc., vol. 25, pp. 1562-1565, 2003.

[114] M. G. Catalano, G. Grioli, E. Farnioli, a. Serio, C. Piazza, and a. Bicchi, "Adaptive synergies for the design and control of the Pisa/IIT SoftHand," Int. J. Robot. Res. , vol. 33, no. 5, pp. 768-782, 2014.

[115] C. Cipriani, M. Controzzi, and M. C. Carrozza, "The SmartHand transradial prosthesis.," J. Neuroeng. Rehabil., vol. 8, no. 1, p. 29, 2011.

[116] M. Grebenstein, A. Albu-Schäffer, T. Bahls, M. Chalon, O. Eiberger, W. Friedl, R. Gruber, S. Haddadin, U. Hagn, R. Haslinger, H. Höppner, S. Jörg, M. Nickl, A. Nothhelfer, F. Petit, J. Reill, N. Seitz, T. Wimböck, S. Wolf, T. Wüsthoff, and G. Hirzinger, "The DLR hand arm system," Proc. - IEEE Int. Conf. Robot. Autom., pp. 3175-3182, 2011.

[117] M. Ortiz-Catalan, F. Rouhani, R. Branemark, and B. Hakansson, "Offline accuracy: A potentially misleading metric in myoelectric pattern recognition for prosthetic control," Proc. Annu. Int. Conf. IEEE Eng. Med. Biol. Soc. EMBS, vol. 2015Novem, pp. 1140-1143, 2015. 
[118] M. R. Ahsan, M. I. Ibrahimy, and O. O. Khalifa, "Advances in Electromyogram Signal Classification to Improve the Quality of Life for the Disabled and Aged People," J. Comput. Sci., vol. 6, no. 7, pp. 706-715, Jul. 2010.

[119] L. Liu, P. Liu, E. a. Clancy, E. Scheme, and K. B. Englehart, "Electromyogram whitening for improved classification accuracy in upper limb prosthesis control," IEEE Trans. Neural Syst. Rehabil. Eng., vol. 21, no. 5, pp. 767-774, 2013.

[120] K. Englehart, B. Hudgins, P. a Parker, and M. Stevenson, "Classification of the myoelectric signal using time-frequency based representations," Med. Eng. Phys., vol. 21, no. 6-7, pp. 431-8, 1999.

[121] L. Hargrove, Ping Zhou, K. Englehart, and T. A. Kuiken, "The Effect of ECG Interference on Pattern-Recognition-Based Myoelectric Control for Targeted Muscle Reinnervated Patients," IEEE Trans. Biomed. Eng., vol. 56, no. 9, pp. 2197-2201, Sep. 2009.

[122] G. Li, A. E. Schultz, and T. A. Kuiken, "Quantifying Pattern Recognition - Based Myoelectric Control of Multifunctional Transradial Prostheses," IEEE Trans. Neural Syst. Rehabil. Eng., vol. 18, no. 2, pp. 185-192, Apr. 2010.

[123] M. Ortiz-Catalan, B. Hakansson, and R. Branemark, "An osseointegrated human-machine gateway for long-term sensory feedback and motor control of artificial limbs," Sci. Transl. Med., vol. 6, no. 257, p. 257re6-257re6, 2014.

[124] D. M. Oleske and J. J. Hahn, "Work-related injuries of the hand: data from an occupational injury/illness surveillance system.," J. Community Health, vol. 17, pp. 205-219, 1992.

[125] J. Hussmann, J. Kucan, and R. Russell, "Electrical treatment injuries - morbidity, outcome and treatment rationale," Burns, vol. 21, no. 7, pp. 530-535, 1995.

[126] S. Waikakul, "Revascularization of degloving injuries of the limbs," Injury, vol. 28, no. 4, pp. 271-274, 1997.

[127] H. P. von Schroeder and M. J. Botte, "Crush syndrome of the upper extremity," Hand Clin., no. 14, pp. 451-6, 1998.

[128] S. Togawa, N. Yamami, H. Nakayama, Y. Mano, K. Ikegami, and S. Ozeki, "The validity of the mangled extremity severity score in the assessment of upper limb injuries.," J. Bone Joint Surg. Br., vol. 87, no. 11, pp. 1516-9, Nov. 2005.

[129] H. Chim, M. A. Maricevich, B. T. Carlsen, S. L. Moran, C. J. Salgado, F.-C. Wei, and S. Mardini, "Challenges in replantation of complex amputations.," Semin. Plast. Surg., vol. 27, no. 4, pp. 182-9, Nov. 2013. 
[130] S.-H. Wang, K.-F. Young, and J.-N. Wei, "Replantation of severed limbs-Clinical analysis of 91 cases," J. Hand Surg. Am., vol. 6, no. 4, pp. 311-318, Jul. 1981.

[131] R. C. Russell, B. M. O'Brien, W. a. Morrison, G. Pamamull, and A. MacLeod, “The late functional results of upper limb revascularization and replantation.," J. Hand Surg. Am., vol. 9, no. 5, pp. 623-33, Sep. 1984.

[132] M. J. Bosse, E. J. MacKenzie, J. F. Kellam, A. R. Burgess, L. X. Webb, M. F. Swiontkowski, R. W. Sanders, A. L. Jones, M. P. McAndrew, B. M. Patterson, M. L. McCarthy, T. G. Travison, and R. C. Castillo, "An analysis of outcomes of reconstruction or amputation after leg-threatening injuries.," N. Engl. J. Med., vol. 347, no. 24, pp. 1924-1931, 2002.

[133] R. Sherman, "To reconstruct or not to reconstruct?," N. Engl. J. Med., no. 347, pp. 1906-7, 2002.

[134] K. Tsuge, "Treatment of established Volkmann's contracture.," J. Bone Joint Surg. Am., vol. 57, no. 7, pp. 925-9, Oct. 1975.

[135] J. M. Serletti, A. J. Carras, R. J. O’Keefe, and R. N. Rosier, "Functional outcome after soft-tissue reconstruction for limb salvage after sarcoma surgery," Plast. Reconstr. Surg., vol. 102, 1998.

[136] A. D. Roche, H. Rehbaum, D. Farina, and O. C. Aszmann, "Prosthetic Myoelectric Control Strategies: A Clinical Perspective," Curr. Surg. Reports, vol. 2, no. 3, p. 44, Jan. 2014.

[137] R. C. Lyle, "A performance test for assessment of upper limb function in physical rehabilitation treatment and research," vol. 4, no. 4. pp. 483-492, 1981.

[138] P. L. Hudak, P. C. Amadio, and C. Bombardier, "Development of an upper extremity outcome measure: the DASH (disabilities of the arm, shoulder and hand) [corrected]. The Upper Extremity Collaborative Group (UECG)," American journal of industrial medicine, vol. 29, no. 6. pp. 602-8, 1996.

[139] J. E. Ware and B. Gandek, "Overview of the SF-36 Health Survey and the International Quality of Life Assessment (IQOLA) Project.," J. Clin. Epidemiol., vol. 51, no. 11, pp. 903-12, Nov. 1998.

[140] H. Matsuzaki, H. Narisawa, H. Miwa, and S. Toishi, "Predicting functional recovery and return to work after mutilating hand injuries: Usefulness of Campbell's Hand Injury Severity Score," J. Hand Surg. Am., vol. 34, no. 5, pp. 880-885, 2009.

[141] B. D. Medling, R. A. Bueno, R. C. Russell, and M. W. Neumeister, "Replantation outcomes," Clin. Plast. Surg., vol. 34, no. 2, pp. 177-185, 2007. 
[142] S. Raspopovic, M. Capogrosso, F. M. Petrini, M. Bonizzato, J. Rigosa, G. Di Pino, J. Carpaneto, M. Controzzi, T. Boretius, E. Fernandez, G. Granata, C. M. Oddo, L. Citi, A. L. Ciancio, C. Cipriani, M. C. Carrozza, W. Jensen, E. Guglielmelli, T. Stieglitz, P. M. Rossini, and S. Micera, "Restoring Natural Sensory Feedback in Real-Time Bidirectional Hand Prostheses," Sci. Transl. Med., vol. 6, no. 222, p. 222ra19-222ra19, 2014.

[143] G. Courtine, S. Micera, J. DiGiovanna, and J. D. R. Millán, "Brain-machine interface: closer to therapeutic reality?," Lancet, vol. 381, no. 9866, pp. 515-7, Mar. 2013.

[144] T. a Kung, N. B. Langhals, D. C. Martin, P. J. Johnson, P. S. Cederna, and M. G. Urbanchek, "Regenerative peripheral nerve interface viability and signal transduction with an implanted electrode.," Plast. Reconstr. Surg., vol. 133, no. 6, pp. 1380-94, 2014.

[145] M. GROB, N. A. PAPADOPULOS, A. ZIMMERMANN, E. BIEMER, and L. KOVACS, "The Psychological Impact of Severe Hand Injury," J. Hand Surg. (European Vol., vol. 33, no. 3, pp. 358-362, 2008.

[146] W. K. Ng, M. N. Kaur, and A. Thoma, "Long-term outcomes of major upper extremity replantations.," Can. J. Plast. Surg., vol. 22, no. 1, pp. 9-13, Jan. 2014.

[147] L. Kovacs, M. Grob, A. Zimmermann, M. Eder, P. Herschbach, G. Henrich, R. Zimmer, E. Biemer, and N. a Papadopulos, "Quality of life after severe hand injury.," J. Plast. Reconstr. Aesthet. Surg., vol. 64, no. 11, pp. 1495-502, Nov. 2011.

[148] T. M. Meyer, "Psychological aspects of mutilating hand injuries," Hand Clin., vol. 19, no. 1, pp. 41-49, 2003.

[149] W. W. Weddington, K. B. Segraves, and M. A. Simon, "Psychological outcome of extremity sarcoma survivors undergoing amputation or limb salvage.," J. Clin. Oncol., vol. 3, no. 10, pp. 1393-9, Oct. 1985.

[150] a Mayer, K. Kudar, K. Bretz, and J. Tihanyi, "Body schema and body awareness of amputees.," Prosthet. Orthot. Int., vol. 32, no. 3, pp. 363-382, 2008.

[151] F. A. Muthny, References for evaluation scales in quality assurance in rehabilitation-- 6. Assessment of coping processes with the Freiburg Questionnaire of Illness Coping. Rehabilitation (Stuttg), 1996.

[152] D. Farina and O. Aszmann, "Bionic limbs: clinical reality and academic promises.," Sci. Transl. Med., vol. 6, no. 257, p. 257ps12, Oct. 2014.

[153] G. S. Dhillon, S. M. Lawrence, D. T. Hutchinson, and K. W. Horch, "Residual function in peripheral nerve stumps of amputees: implications for neural control of artificial limbs.," J. Hand Surg. Am., vol. 29, no. 4, pp. 605-15-8, Jul. 2004. 
[154] B. Wodlinger and D. M. Durand, "Peripheral nerve signal recording and processing for artificial limb control," 2010 Annu. Int. Conf. IEEE Eng. Med. Biol. Soc. $E M B C^{\prime} 10$, pp. 6206-6209, 2010.

[155] V. Gilja, P. Nuyujukian, C. A. Chestek, J. P. Cunningham, B. M. Yu, J. M. Fan, M. M. Churchland, M. T. Kaufman, J. C. Kao, S. I. Ryu, and K. V Shenoy, "A highperformance neural prosthesis enabled by control algorithm design.," Nat. Neurosci., vol. 15, no. 12, pp. 1752-7, 2012.

[156] J. P. Donoghue, "Connecting cortex to machines: recent advances in brain interfaces.," Nat. Neurosci., vol. 5 Suppl, pp. 1085-8, 2002.

[157] J. E. Cheesborough, G. A. Dumanian, L. H. Smith, and T. A. Kuiken, "Targeted Muscle Reinnervation and Advanced Prosthetic Arms," Semin. Plast. Surg., vol. 1, no. 212, pp. 62-72, 2015.

[158] P. Zhou, M. M. Lowery, K. B. Englehart, H. Huang, G. Li, L. Hargrove, J. P. A. Dewald, and T. A. Kuiken, "Decoding a new neural machine interface for control of artificial limbs.," J. Neurophysiol., vol. 98, no. 5, pp. 2974-82, 2007.

[159] F. Negro, A. Holobar, and D. Farina, "Fluctuations in isometric muscle force can be described by one linear projection of low-frequency components of motor unit discharge rates," J. Physiol., vol. 587, no. 24, pp. 5925-5938, 2009.

[160] E. a Clancy and N. Hogan, "Probability density of the surface electromyogram and its relation to amplitude detectors.," IEEE Trans. Biomed. Eng., vol. 46, no. 6, pp. 730-9, Jun. 1999.

[161] F. Negro, S. Muceli, A. M. Castronovo, A. Holobar, and D. Farina, "Multi-channel intramuscular and surface EMG decomposition by convolutive blind source separation," J. Neural Eng., vol. 13, no. 2, p. 26027, Apr. 2016.

[162] D. Atkins, D. Heard, and W. Donovan, "Epidemiologic overview of individuals with upper-limb loss and their reported research priorities," JPO J. Prosthetics Orthot., vol. 8, no. 1, 1996.

[163] C. Castellini and M. Nowak, "EMG-Based Prediction of Multi-DOF Activations Using Single-DOF Training: A Preliminary Result," Myoelectric Controls Symposium (MEC). pp. 45-49, 2014. 


\section{Appendix I - Multichannel transradial socket design}

Myoelectric transradial systems containing more than two electrodes are still not freely available on the market. Therefore, procedures for producing sockets needed for conducting studies presented in this thesis have not been yet established and are not part of regular activities of orthopaedic technicians. For the purpose of the studies conducted here over 20 semi-permanent sockets have been custom designed and made for more than 15 impaired individuals.

Brief conceptual design and technical notes are given in this appendix as a future reference. The details presented are far from ideal and further improvements are planned and needed. In addition, it should be noted that the presented concept was developed specifically with Ottobock's Michelangelo Hand in mind, equipped with standard rotation and experimental flexion/extension unit. Additionally, sockets were made in such way so that they can host up to eight Ottobock surface EMG electrodes of either kind. However, with minor adjustments, this design can be adapted for other prosthetic solutions as well.

In order to secure a snug fit of the socket, any access soft tissue and prominent scarring that can be observed on the stump should be noted. A plan for dealing with potential loose skin is an absolute must and critical areas should be marked ensuring a firm and reliable mold sampling. This procedure is a fairly standard one and requires technician to have an average level of skill. For the purpose of tight fitting and easy removal of negative mold, plastic foil should be neatly wrapped around the stump in not more than three layers. First layer of medical plaster cast needs to be lightly soaked and used to quickly yet firmly cover the entire region of the forearm and up to one third of the upper arm. Region around the elbow usually needs to be additionally strengthened. At this point, while the plaster is still soft, a firm pressure has to be applied using thumb and middle finger distributing the grip force on the back side of the elbow between the two epicondyles. If done properly, all the prominent landmarks of this region should be enhanced and comfortable socket fit is guaranteed.

At this point, the foundation is set for applying the second layer of plaster made out of cellacast, which will provide the necessary sturdiness of the negative. Same as before, special care needs to be given to the elbow region. In order to have easier time in removing the applied layers from the subject's stump, it is recommended to wrap cellacast around one centimetre shorter along the upper arm than the medical plaster. 
Once the desired level of hardness is achieved, negative cast should be removed by making two incisions on both sides of the upper arm all the way to the forearm. An example of a negative is shown in Figure 18.

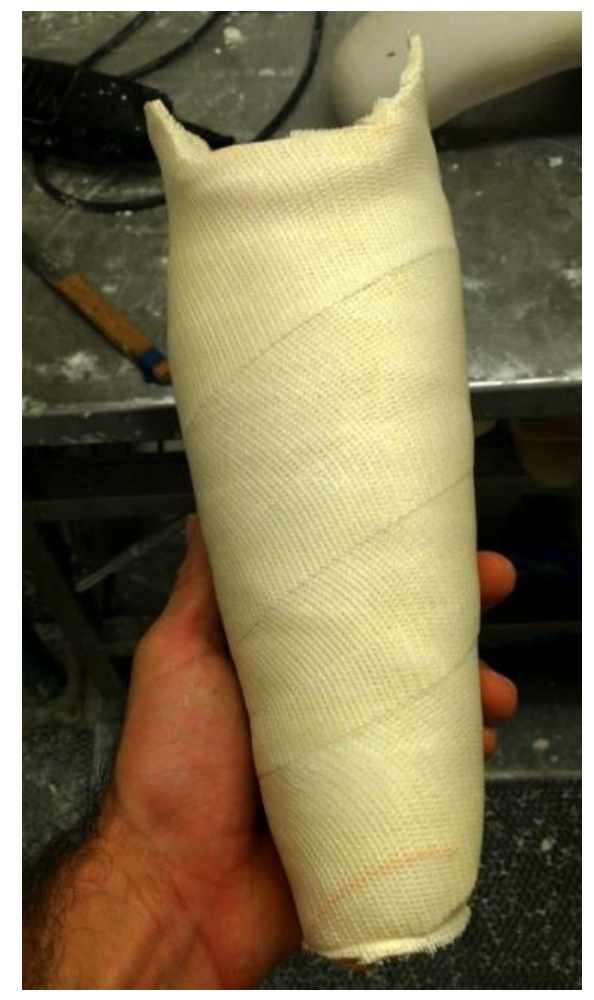

Figure 18 - An example of a negative cast with the second layer made out of cellecast. This particular patient has a rather long and strong stump which enables easy and neat sampling.

Production of a positive is almost the same as in any other socket design requiring the negative to be filled with a mixture of fine cement and water allowing smooth molding and easy removal from the plaster. Once the cement is hardened, negative cast needs to be removed and the general surface of the obtained stump positive should be polished (Figure 19). Special care of prominent landmarks as well as thorough grinding of the upper arm region needs to be done and at this point it is of outmost importance that the socket "end line" is enhanced and smoothly defined as it will highly reflect on the subject's comfort. This stage additionally requires that the electrode positions are marked and appropriately flattened down. Using a previously determined paper template with exact sizes and positions of the sensors helps in this process. In addition, a centre axis point of the tip of the stump should also be marked determining the appropriate fitting of the exhaust anchoring point. 


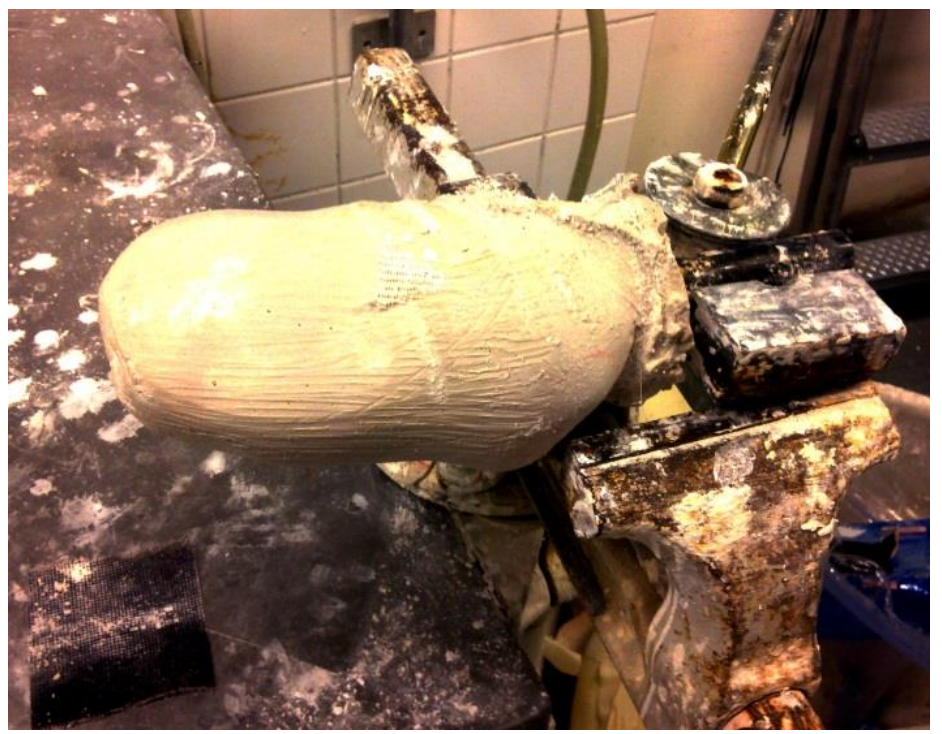

Figure 19 - Positive cast after molding ready for refinement and polishing. Strong anchoring line across the front of the elbow can be observed.

Once the polishing of the positive is done to a satisfactory level, a rapid socket liner can be made. First, the cast needs to be wrapped into temperature resilient foil or textile mash, allowing easy separation of the highly warmed up $\left(\sim 1200^{\circ} \mathrm{C}\right)$ thermoplastic liner material. As soon as the thermoplast is applied all the access air is vacuumed out securing the tight following of the contours defined on the positive (Figure 20). This is a critical point of the process, since too rapid air extraction might cause the unwanted folding of the material. Albeit, if the vacuuming process is too slow it can lead to gap and bubble formation resulting in a loos or uncomfortable fit.

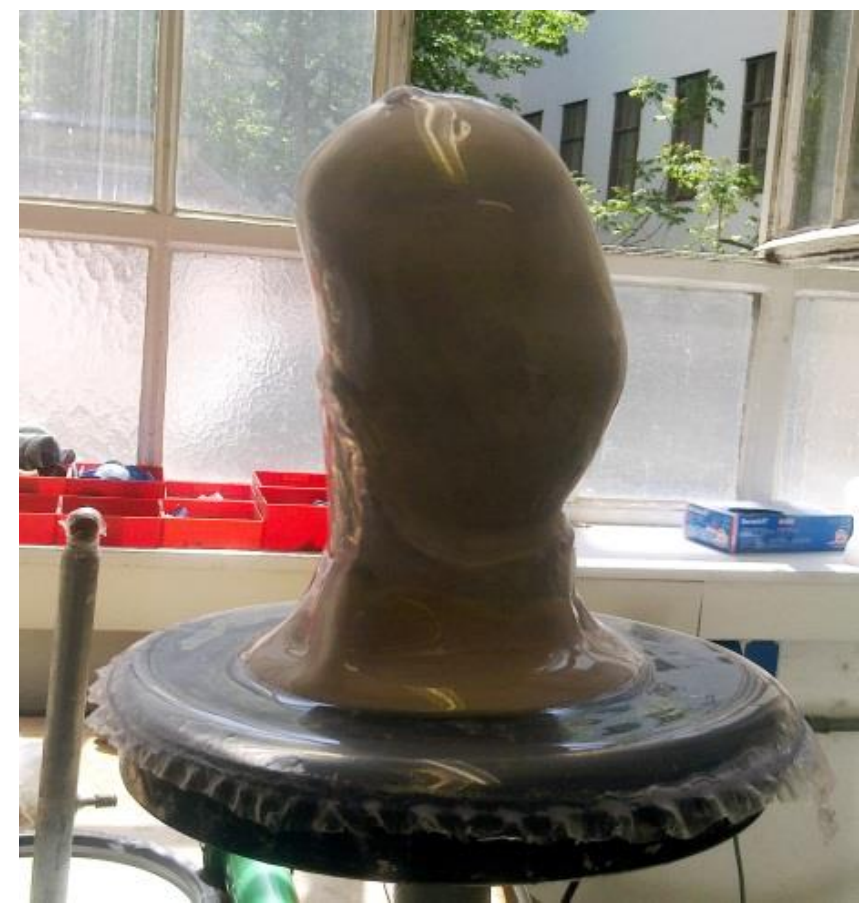

Figure 20 - Warmed up thermoplast has to be carefully applied on the positive in order to ensure tight and comfortable fit. This is being done using a vacuum pump during gentle and slow stretching of the material. 
Upon the removal of the liner, some trimming and subtle polishing should be done in order to remove the access plastic around the edges. Hard rubber rotating chisel can be used for making the holes for the electrodes on, now clearly visible, markings and similar should be done for the fitting exhaust. First test fitting of the patient should be performed at this stage. In case of discomfort, still some minor changes can be made at this point by targeted reheating and remolding of the material.

Once satisfied with the fit, depending on the size and shape of the stump the outer semi-permanent socket can be made. If the stump is very short or narrow, a hard polyurethane extension can be made on the top of the positive mold holding the liner so that it gives a solid structure for application of a fresh layer of cellacast (Figure 21). This layer will then be used as a chassis for mounting of the lamination ring and guiding the fitting exhaust. When dry, outer cast needs to be removed, cleaned, trimmed and provided with the screw holes which will secure the connection to the liner.

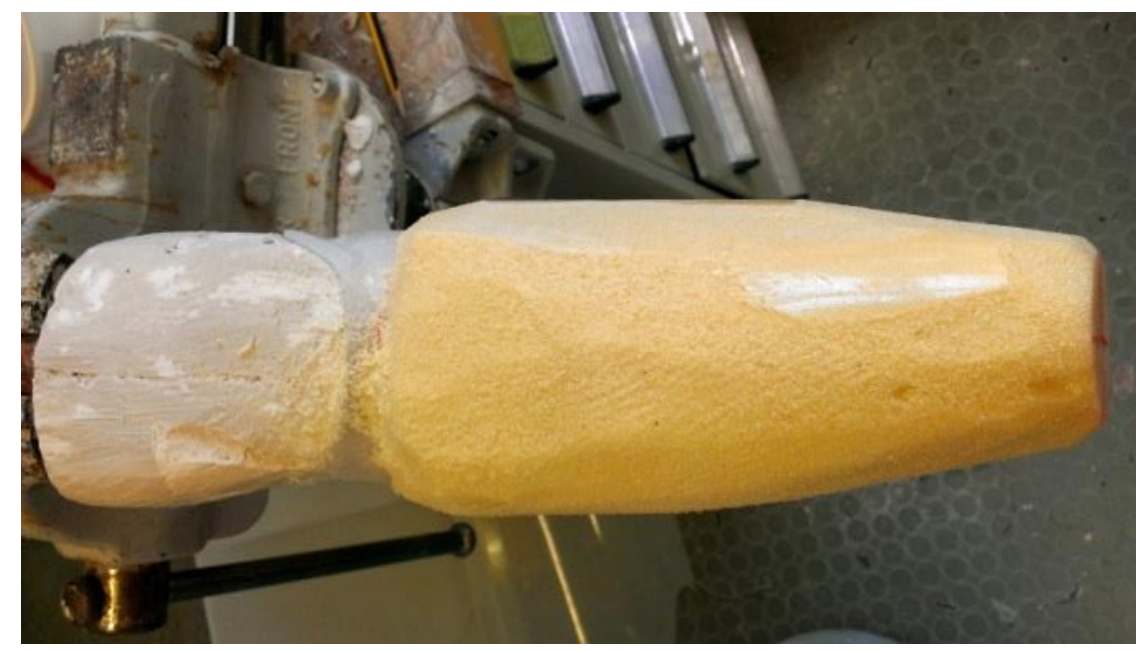

Figure 21 - Positive cast with a thermoplastic liner still attached and polished polyurethane extension pre-shaped to provide a solid base for casting the outer semi-permanent socket

In case of longer stumps, an open design of the socket is recommended, as in Figure 22 , promoting easy donning and doffing. In this way, standard fitting vacuum problems are also avoided. Open design sockets usually are not well suited for standard lamination rings, therefore a 3D printed carrier can be mounted on the distal part of the outer socket. 


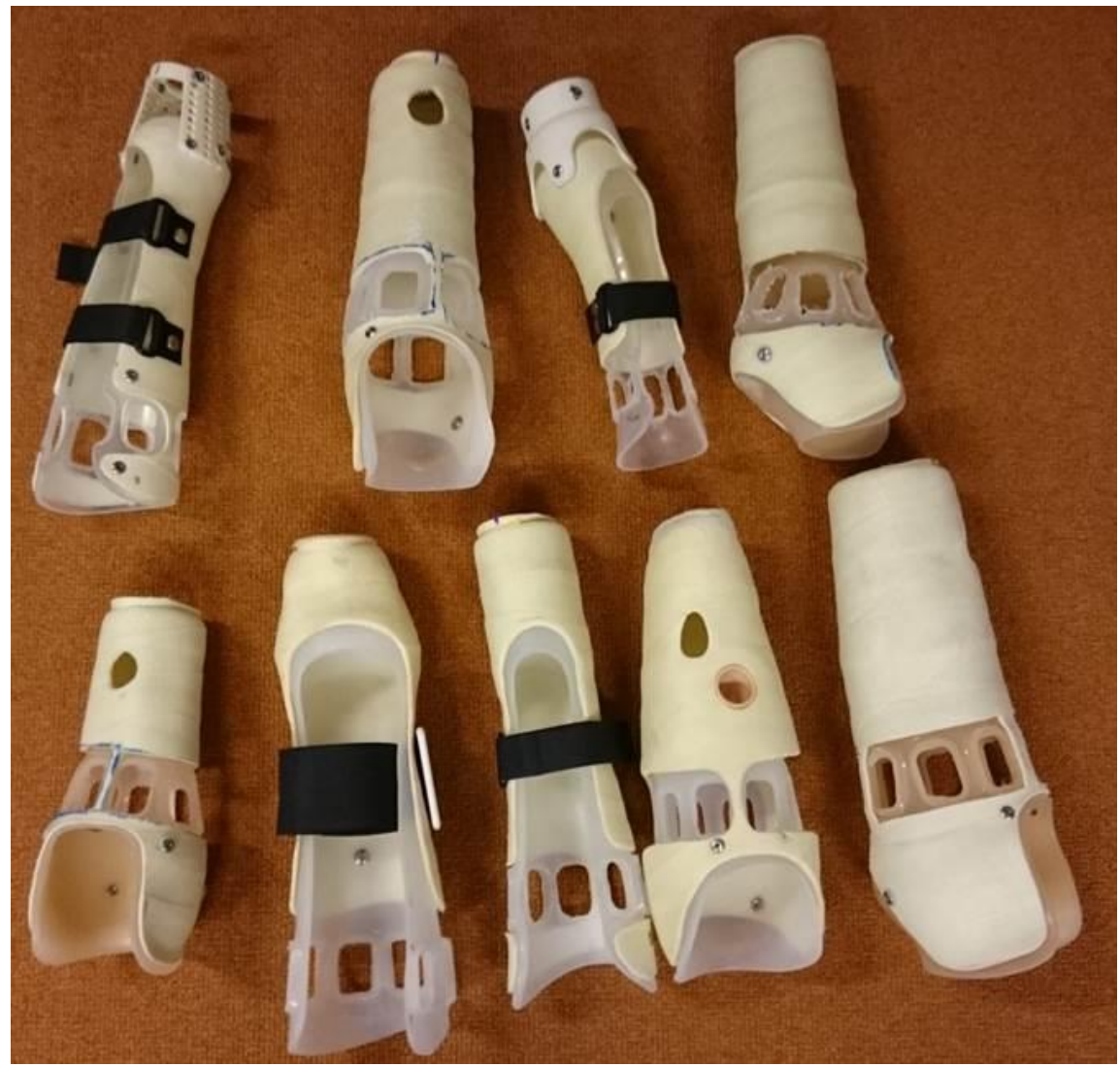

Figure 22 - Examples of different semi-permanent sockets built using the presented technique. Four sockets featuring the black Velcro strips are so called open designs intended for users with transcarpal amputations or generally longer stumps

Finally, electrodes can be attached using a rubber band hosting 3D printed electrode housings which allows adjustable fitting and tension distribution. This has proven to be a good solution for compensating slight stump volume changes and intrinsic muscle movements during contractions. 


\section{Appendix II - Simultaneous and proportional myocontrol algorithm}

\section{This appendix is based on the following publication*:}

Amsuess, S., Vujaklija, I., Gobel, P., Roche, A., Graimann, B., Aszmann, O., et al. (2015). Context-Dependent Upper Limb Prosthesis Control for Natural and Robust Use. IEEE Trans. Neural Syst. Rehabil. Eng., 1-1. doi:10.1109/TNSRE.2015.2454240.

*This paper is core of the PhD thesis of the first author. I contributed as second author to the developments of the algorithm and to the experimental tests and data analysis. The study and paper are not included as a main study but as an appendix as they are part of the cited PhD thesis. An appendix on this paper is, however, provided since this work is at the basis of the subsequent developments described as the core of the present thesis.

Advanced myoelectric control algorithm used throughout this thesis is an adaptation of the approach described in [67]. Due to its extensive use and pivotal point in testing some of the presented hypotheses, this control paradigm, which we have developed in parallel to the presented work, is further discussed in this appendix.

The main goal of this advanced control system was to provide users with more natural control. Based on a survey [162] of current myoelectric users, simultaneous activation of two joints ranked quite high on a wish list of desired prosthetic improvements. Considering that in general, during reaching tasks we tend to first perform gross prepositioning of our entire arm and only then perform the final fine act of grasping, this was set as a main design goal while developing the system.

Taking into account the design experiences and the developments of similar systems in the past, pros and cons of simultaneous and sequential estimators have been observed. Namely, the first ones are able to deliver intuitive, simultaneous control over dynamic activations, while the later ones are suited for precise, single DoF control over many motions in a reasonably robust way. This has led to an idea of a hybrid system which would be capable of combining the best of both worlds while not compromising the strong points of either. An idea of the two parallel estimators working exclusively as needed has been proposed (Figure 23). 


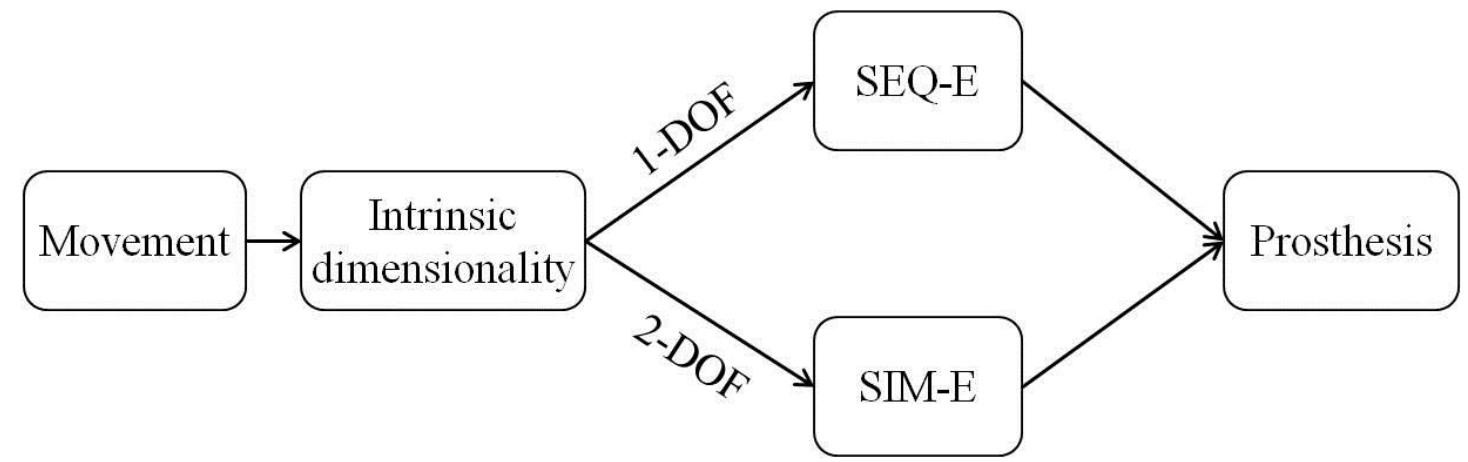

Figure 23 - Hybrid myoelectric control algorithm capable of estimating simultaneous and proportional movements. Based on intrinsic dimensionality of the movement, new incoming data point will be forwarded to either sequential estimator (SEQ-E) in case of the single DoF activation or to the simultaneous estimator (SIM-E) if the processed EMG is a product of a multiple DoF activation. (C) 2015|IEEE TNSRE

The first point which had to be carefully considered was the determination of the intrinsic dimensionality of the incoming data sample. More precisely, the overall system had to obtain knowledge whether the observed EMG is a result of an individual or simultaneous motion. Simple yet effective measure has been taken at this point relaying on a pre-set threshold. Once the training data for all the single motions has been collected, a feature space can be formed resulting in the clustering of the points originating from the same motion. New unseen feature then lands into the formed space and based on its distance from each of the point clouds a sturdy decision on whether it belongs to a single or simultaneous motion can be made. Basically, if it fits into one of the clouds or in a near proximity of it, system should classify it as a single DoF activation, yet if this is not the case it is going to be treated as a multiple DoF motion. This is a reasonable assumption considering that a feature vector containing the information from the two distinct movement classes lies somewhere between those two classes [163].

In order to measure the distance between the new point and the training clouds, after exhausting testing, a simple Mahalanobis distance measure proved itself to be the best trade-off between the accuracy of made decisions and computation time:

$$
D_{\text {Mahal }}=\left(\boldsymbol{x}-\boldsymbol{\mu}_{i}\right)^{T} \Sigma_{i}^{-1}\left(\boldsymbol{x}-\boldsymbol{\mu}_{i}\right)
$$

where $\boldsymbol{x}$ is a feature vector, $i$ is a given class with its mean vector $\boldsymbol{\mu}_{i}$ and the covariance matrix $\Sigma_{i}$. Though, it should be noted that the threshold for determining whether the new data point is novel, and as such originating from a simultaneous activation, has to be set manually in this case.

The rest of the system was implemented as shown in Figure 23. As the sequential estimator, a Common Spatial Pattern Proportional Estimator (CSP-PE) [52] was chosen. CSP is in essence a supervised spatial filtering method which aims at maximally discriminating between data stemming from two differentiable classes. This is being achieved by using a linear weighting of the input signals from different channels. 
Considering, it performs a linear transformation of an input signal $x \in \mathbb{R}^{n}$ using a weighting matrix $W \in \mathbb{R}^{n x m}$ on $m$-dimensional vector $y \in \mathbb{R}^{m}$ :

$$
y=W^{T} x
$$

If both $x$ and $y$ have been drawn from centred distributions, and if $E[\cdot]$ is the expectation operator and $\hat{\Sigma}$ is empiric covariance matrix of $x$, then the variance of $y$ can be obtained as:

$$
\operatorname{var}(y)=E[y y]^{T}=W^{t} E[x x]^{T} W=W^{T} \hat{\Sigma} W
$$

In order to obtain the optimal weighting matrix $W$ the generalized Rayleigh quotient is maximized. This basically maximizes the variance of the output for data from class 1 , while at the same time it minimizes it for the data from class 2 :

$$
W:=\arg _{W} \max W^{T} \widehat{\Sigma}_{1} W\left(W^{T} \widehat{\Sigma}_{2} W\right)^{-1}
$$

with $\hat{\Sigma}_{1}$ and $\hat{\Sigma}_{2}$ representing the empiric covariance matrices for the classes 1 and 2 respectively. Maximization of the previous expression with respect to $W$ can be achieved by applying the Lagrangian method through generalized eigenvalue decomposition of $\hat{\Sigma}_{2}^{-1} \widehat{\Sigma}_{1}$.

For this problem to be well defined, scaling of $W$ has to be fixed. That can be done by setting the norm of $W$ to $1:\|W\|^{2}=W^{T} W=1$, which results in the constrained optimization problem:

$$
\arg _{W} \max W^{T} \widehat{\Sigma}_{1} W\left(W^{T} \hat{\Sigma}_{2} W\right)^{-1} \text { subject to } W^{T} W=1
$$

This is known as quadratic programing and in order to obtain a closed form solution (19) can be transformed into Lagrangian formulation $\mathcal{L}(W)$

$$
\mathcal{L}(W)=W^{T} \widehat{\Sigma}_{1} W\left(W^{T} \widehat{\Sigma}_{2} W\right)^{-1}-\lambda\left(W^{T} W-1\right)
$$

where $\lambda$ are Lagrange multipliers. If $(20)$ is differentiated with respect to $W$ and equalized to 0 it reads:

$$
\begin{aligned}
\frac{\partial \mathcal{L}(W)}{\partial W} & =2 W \hat{\Sigma}_{2}^{-1} \widehat{\Sigma}_{1}-2 \lambda W=0 \\
& \rightarrow W \hat{\Sigma}_{2}^{-1} \widehat{\Sigma}_{1}=\lambda W
\end{aligned}
$$

(22) is true for all tuples $(W, \lambda)$ where $W \in \mathcal{W}$ and $\lambda \in \mathbb{R}$ and $\mathcal{W}$ is the set of eigenvectors of $\hat{\Sigma}_{2}^{-1} \hat{\Sigma}_{1}$ and $\lambda$ the corresponding eigenvalues. The quantity of $\lambda$ is a measure of separation quality for its corresponding $W$. When sorting the obtained generalized eigenvectors according to their respective eigenvalues from largest to lowest, the first vector constitutes the optimal spatial filter which maximizes the 
variance of $y$ for the data of class 1 while minimizing it for the data of class 2 . The last vector operates in the same way for the data of class 2 .

Once this approach is applied on a feature vector, the obtained operator ends up linearly combining the EMG features which, if proportional to the exerted force, result in delivering a transformation that combines feature values across all channels. Weighted average using CSP can be extended to obtain the maximized ratio of activation between all involved competing classes by calculating the separation through optimization of weighting matrices on one against one basis among all the possible class pairs. This finally results in a regression method which correlates EMG activation and the target output (velocity or force of the prosthesis) while at the same time it promotes the maximum separation between the movement classes. This approach has been shown to outperform classical LDA classification during online control of a physical prosthesis [52].

For the simultaneous estimator, linear regression was selected. Since linear regression was shown to work well with 2 DOF only [57], control of wrist rotation and wrist flexion/extension was done using this estimator for natural positioning of the hand. CSP-PE was capable of controlling all functions of the prosthesis individually.

Practical pilot tests have shown that the border between the single and the multiple activations should not be hard step like function, but rather smooth sigmoidal one. This is mostly due to the noise present in the system. It should be noted that errors in the estimation of the intrinsic movement dimensionality did not automatically result in a wrong activation of the prosthesis. It only implied that an intended simultaneous movement was performed sequentially or vice-versa. Since unintended simultaneous movements were more detrimental to the control than unintended sequential movements, the manually set threshold for the Mahalanobis distance approach was selected to slightly favour the sequential estimation.

For the purpose of the online feasibility evaluation, series of test scenarios have been devised and conducted. Two transradial amputees were fitted with a prosthetic devise previously described in chapter 2.1. Besides BBT, CPRT and SHAP tests, a custom designed block turn test was used for evaluating performance of the proposed system. This custom test was intended to enforce the users to activate all available movements (3.5 DoF) of the prostheses in order to ensure that the full provided functionality is available. Namely, subjects were prompted to pick up a rectangular wooden block which was lying on a shelf at shoulder level, rotate it and place it in the upward position at the lower shelf at the hip level. In order to conclude the task, block was supposed to be picked up one last time and placed horizontally at the same shelf level. The time of each successful execution as well as the number of drops was recorded over three trials. In addition to these two patients, ten able bodied volunteers were recruited and fitted with a hybrid device in order to execute all tests but SHAP. 
As a conclusion, these experiments have indicated high potential of the proposed approach and a possibility of its integration into a reasonable prosthetic system. Since all of the participants were able to execute all of the presented tasks, certain level of confidence that the system can be transferred across the population was gained. The same achievement in combination with the reported scores showed a degree of sturdiness of this approach with respect to various environmental factors.

Though, as outlined throughout this thesis, testing of a novel myoelectric system requires larger number of fully fitted amputees which have been systematically evaluated using clinically recognized metrics. Therefore, this particular publication has been focused on communicating the idea of a novel algorithmic concept and its capability to be implemented in a constrained environment such as the one of the presented prosthetic system. The actual performance evaluation was made and presented in different scenarios as a part of this thesis, but only after the rigorous consideration of all clinically relevant parameters. 


\section{Appendix III - Dissemination}

\section{Journal publications:}

1. Farina, D., Rehbaum, H., Holobar, A., Vujaklija, I., Jiang, N., Hofer, C., ... Aszmann, O. (2014). Non-Invasive, Accurate Assessment of the Behavior of Representative Populations of Motor Units in Targeted Reinnervated Muscles. IEEE Transactions on Neural Systems and Rehabilitation Engineering: A Publication of the IEEE Engineering in Medicine and Biology Society, 4320(c). http://doi.org/10.1109/TNSRE.2014.2306000

2. Jiang, N., Rehbaum, H., Vujaklija, I., Graimann, B., \& Farina, D. (2014). Intuitive, Online, Simultaneous, and Proportional Myoelectric Control Over Two Degrees-ofFreedom in Upper Limb Amputees. IEEE Transactions on Neural Systems and Rehabilitation Engineering : A Publication of the IEEE Engineering in Medicine and Biology Society, 22(3), 501-10. http://doi.org/10.1109/TNSRE.2013.2278411

3. Jiang, N., Vujaklija, I., Rehbaum, H., Graimann, B., \& Farina, D. (2014). Is accurate mapping of EMG signals on kinematics needed for precise online myoelectric control? IEEE Transactions on Neural Systems and Rehabilitation Engineering : A Publication of the IEEE Engineering in Medicine and Biology Society, 22(3), 549-58. http://doi.org/10.1109/TNSRE.2013.2287383

4. Amsuess, S., Vujaklija, I., Gobel, P., Roche, A., Graimann, B., Aszmann, O., \& Farina, D. (2015). Context-Dependent Upper Limb Prosthesis Control for Natural and Robust Use. IEEE Transactions on Neural Systems and Rehabilitation Engineering, 1-1. http://doi.org/10.1109/TNSRE.2015.2454240

5. Hofmann, D., Jiang, N., Vujaklija, I., \& Farina, D. (2015). Bayesian Filtering of Surface EMG for Accurate Simultaneous and Proportional Prosthetic Control. IEEE Transactions on Neural Systems and Rehabilitation Engineering, 1-1. http://doi.org/10.1109/TNSRE.2015.2501979

6. Ison, M., Vujaklija, I., Whitsell, B., Farina, D., \& Artemiadis, P. (2015). High-Density Electromyography and Motor Skill Learning for Robust Long-Term Control of a 7DoF Robot Arm. IEEE in Transactions on Neural Systems \& Rehabilitation Engineering, to appear, 1-10.

7. Roche, A. D., Vujaklija, I., Amsüss, S., Sturma, A., Göbel, P., Farina, D., \& Aszmann, O. C. (2015). A Structured Rehabilitation Protocol for Improved Multifunctional Prosthetic Control: A Case Study. Journal of Visualized Experiments : JoVE, (105), e52968. http://doi.org/10.3791/52968

8. Kapelner, T., Jiang, N., Holobar, A., Vujaklija, I., Roche, A. D., Farina, D., \& Aszmann, O. C. (2016). Motor Unit Characteristics after Targeted Muscle Reinnervation. Plos One, 11(2), e0149772. http://doi.org/10.1371/journal.pone.0149772 
9. Vujaklija, I., Farina, D., \& Aszmann, O. (2016). New developments in prosthetic arm systems. Orthopedic Research and Reviews, Volume 8, 31-39. http://doi.org/10.2147/ORR.S71468

10. Aszmann, O. C., Vujaklija, I., Roche, A. D., Salminger, S., Herceg, M., Sturma, A., et al. (2016). Elective amputation and bionic substitution restore functional hand use after critical soft tissue injuries. Nat. Sci. Reports.

\section{Conference publications:}

1. Ison, M., Vujaklija, I., Whitsell, B., Farina, D., \& Artemiadis, P. (2015). Simultaneous Myoelectric Control of a Robot Arm using Muscle Synergy-Inspired Inputs from High-Density Electrode Grids. In IEEE International Conference on Robotics and Automation (pp. 6469-6474). http://doi.org/10.1109/ICRA.2015.7140108

2. Kapelner, T., Jiang, N., Vujaklija, I., Aszmann, O. C., \& Farina, D. (2015). Classification of Motor Unit Activity Following Targeted Muscle Reinnervation. In IEEE engineering in medicine and biology magazine : the quarterly magazine of the Engineering in Medicine \& Biology Society (pp. 22-24).

3. Vujaklija, I., Amsuess, S., Roche, A. D., Farina, D., \& Aszmann, O. C. (2017). Clinical Evaluation of a Socket-Ready Naturally Controlled Multichannel Upper Limb Prosthetic System (pp. 3-7). http://doi.org/10.1007/978-3-319-46532-6_1

4. Muceli, S., Vujaklija, I., Jiang, N., Amsuess, S., Graimann, B., Aszmann, O. C., \& Farina, D. (2017). A Biologically-Inspired Robust Control System for Myoelectric Control (pp. 975-979). http://doi.org/10.1007/978-3-319-46669-9_158

5. Vujaklija, I., Muceli, S., Bergmeister, K., Aszmann, O. C., \& Farina, D. (2017). Prospects of Neurorehabilitation Technologies Based on Robust Decoding of the Neural Drive to Muscles Following Targeted Muscle Reinnervation (pp. 13591363). http://doi.org/10.1007/978-3-319-46669-9_222

\section{Presentations, workshops and lectures:}

Presentation at DEMOVE symposium 2014, Göttingen, Germany "Dexterous robot manipulation using high-density myoelectric control"

Key note lecture at ICORR 2015, Singapore, Singapore "Decoding the neural code of human movements for a new generation of man-machine interfaces"

Workshop at OT bioelettronica Day 2015, Turin, Italy "Advanced myoelectric control of prosthetic devices"

Invited talk at OT World 2016, Leipzig, Germany "Improved hand prosthetic experience through structured training and dexterous control"

Presentation at DEMOVE symposium 2016, Baiona, Spain "Clinical Evaluation of Advanced Upper Limb Myoelectric Prosthetic Systems - importance and challenges" 
Workshop (co-organizer) at Summer School in Neurorehabilitation 2016, Baiona, Spain "Detecting and decoding spinal motor neuron spike trains for understanding the neural control of movement"

Presentation at WeROB 2016, LaGranja, Spain "Clinical Evaluation of a Socket-Ready Naturally Controlled Multichannel Upper Limb Prosthetic System" - Special Session and Best Student Paper Finalist

Presentation at ICNR 2016, LaGranja, Spain "Prospects of Neurorehabilitation Technologies Based on Robust Decoding of the Neural Drive to Muscles following Targeted Muscle Reinnervation"

\section{Teaching activities:}

04/2015 - 07/2015 Georg-August-Universität Göttingen Introduction to Neurorehabilitaion Technologies

2014-2016 Private University of Applied Sciences, Göttingen, Orthobionics Module - Lecture series on Neuroprosthetics

\section{Google Scholar metrics:}

\begin{tabular}{lrr} 
Citation indices & All & Since 2011 \\
\hline Citations & 143 & 143 \\
h-index & 5 & 5 \\
i10-index & 3 & 3
\end{tabular}


Translating Advanced Myocontrol for Upper Limb Prostheses from the Laboratory to Clinics 
Dipl.-Ing. Ivan Vujaklija M.Sc.

Contact Am Vogelsang 1/606

37075 Göttingen, Germany

+49-176-31205512

ivan@vujaklija.rs

\title{
In short
}

PhD student of Human Medical Sciences program at Georg-August-Universität Göttingen; Currently working at Clinic for Trauma Surgery, Orthopaedic Surgery and Plastic Surgery, Research Department of Neurorehabilitation Systems, University of Göttingen as a research assistant. DOB 26/07/1987 Belgrade, Serbia. At the moment residing in Germany.

\section{Education}

10/2013 - to date Georg-August-Universität Göttingen / Universitätsmedizin Göttingen

PhD student at Promotionsprogramm "Humanwissenschaften in der Medizin"

10/2011 - 08/2013 University of Lübeck / University of Applied Sciences Lübeck

International Master's Program "Biomedical Engineering" - M.Sc. Biomedical Eng.

10/2006 - 07/2011 University of Belgrade

Faculty of Electrical Eng., Physical Electronics module, department for biomedical and ecological engineering - Dipl. Eng. of Electrical Eng. and computer sci.

\section{Work Experience}

04/2014 - to date Institute of Neurorehabilitation Systems, Uni. Medical Center Göttingen

Research assistant and a PhD student within the "DEMOVE" project (ERC \#267888)

09/2012 - 04/2014 Ottobock HealthCare GmbH, Duderstadt, Germany

Intern at the "Strategic Technology Management" unit. Conducted research in the areas of myo-control algorithms, biosignal processing and actuated prostheses.

03/2012 - 09/2012 University Hospital Schleswig-Holstein, Campus Lübeck, Germany

Junior research fellow at the Laboratory for Biomechanics and Biomechatronics. Development of framework for stereovision based motion tracking system in subcentimeter volumes and wireless EMG based gait analysis system.

05/2011 - 06/2011 Audiovox d.o.o., Belgrade, Serbia

Intern within the company's technical support with focus on personal hearing aids.

\section{Fellowships}

05/2015 - 12/2015 Universitätsmedizin Wien - CD lab for Restoration of Extremity Function Advanced upper limb prosthetic control and evaluation

06/2014 - 07/2014 Arizona State University - HORC lab, Tempe, AZ, USA

Dexterous robot control using high density EMG

09/2013 - 04/2014 Inst. für Bioprozess- und Analysenmesstechnik, Heilbad Heiligenstadt, Germany Development and assessment of the myoimpedance framework intended to be used as an additional modality in the EMG driven control systems

02/2013 - 04/2014 Department of Neurorehabilitation Engineering, Uni. Medical Center Göttingen Development and assessment of algorithms and techniques for biosignal processing and myoelectric interfaces.

\section{Teaching}

\author{
04/2015 - 07/2015 Georg-August-Universität Göttingen \\ Introduction to Neurorehabilitation Technologies \\ 10/2014 - 01/2016 Private University of Applied Sciences, Göttingen, Orthobionics Module \\ Lecture series on Neuroprosthetics
}




\section{Journal Publications}

Farina, D., Rehbaum, H., Holobar, A., Vujaklija, I., Jiang, N., Hofer, C., ... Aszmann, O. (2014). NonInvasive, Accurate Assessment of the Behavior of Representative Populations of Motor Units in Targeted Reinnervated Muscles. IEEE Transactions on Neural Systems and Rehabilitation Engineering : A Pub.of the IEEE Engineering in Medicine and Biology Society, 4320(c). http://doi.org/10.1109/TNSRE.2014.2306000

Jiang, N., Rehbaum, H., Vujaklija, I., Graimann, B., \& Farina, D. (2014). Intuitive, Online, Simultaneous, and Proportional Myoelectric Control Over Two Degrees-of-Freedom in Upper Limb Amputees. IEEE Transactions on Neural Systems and Rehabilitation Engineering : A Publication of the IEEE Engineering in Medicine and Biology Society, 22(3), 501-10. http://doi.org/10.1109/TNSRE.2013.2278411

Jiang, N., Vujaklija, I., Rehbaum, H., Graimann, B., \& Farina, D. (2014). Is accurate mapping of EMG signals on kinematics needed for precise online myoelectric control? IEEE Transactions on Neural Systems and Rehabilitation Engineering : A Publication of the IEEE Engineering in Medicine and Biology Society, 22(3), 549-58. http://doi.org/10.1109/TNSRE.2013.2287383

Amsuess, S., Vujaklija, I., Gobel, P., Roche, A., Graimann, B., Aszmann, O., \& Farina, D. (2015). ContextDependent Upper Limb Prosthesis Control for Natural and Robust Use. IEEE Transactions on Neural Systems and Rehabilitation Engineering, 1-1. http://doi.org/10.1109/TNSRE.2015.2454240

Hofmann, D., Jiang, N., Vujaklija, I., \& Farina, D. (2015). Bayesian Filtering of Surface EMG for Accurate Simultaneous and Proportional Prosthetic Control. IEEE Transactions on Neural Systems and Rehabilitation Engineering, 1-1. http://doi.org/10.1109/TNSRE.2015.2501979

Ison, M., Vujaklija, I., Whitsell, B., Farina, D., \& Artemiadis, P. (2015). High-Density Electromyography and Motor Skill Learning for Robust Long-Term Control of a 7-DoF Robot Arm. IEEE in Transactions on Neural Systems \& Rehabilitation Engineering, to appear, 1-10.

Roche, A. D., Vujaklija, I., Amsüss, S., Sturma, A., Göbel, P., Farina, D., \& Aszmann, O. C. (2015). A Structured Rehabilitation Protocol for Improved Multifunctional Prosthetic Control: A Case Study. Journal of Visualized Experiments : JoVE, (105), e52968. http://doi.org/10.3791/52968

Kapelner, T., Jiang, N., Holobar, A., Vujaklija, I., Roche, A. D., Farina, D., \& Aszmann, O. C. (2016). Motor Unit Characteristics after Targeted Muscle Reinnervation. Plos One, 11(2), e0149772.

http://doi.org/10.1371/journal.pone.0149772

Vujaklija, I., Farina, D., \& Aszmann, O. (2016). New developments in prosthetic arm systems. Orthopedic Research and Reviews, Volume 8, 31-39. http://doi.org/10.2147/ORR.S71468

O. C. Aszmann, I. Vujaklija, A. D. Roche, S. Salminger, M. Herceg, A. Sturma, L. A. Hruby, A. Pittermann, C. Hofer, S. Amsuess, and D. Farina, "Elective amputation and bionic substitution restore functional hand use after critical soft tissue injuries," Sci. Rep., vol. 6, p. 34960, Oct. 2016.

\section{Skills}

Computer skills

Matlab, Lab View, Nexus, Eagle, pSpice, Biomobius, Visual Studio, C, C++, Fortran, Adobe Photoshop and Premiere, Microsoft Windows and Office

Soft skills

Language skills

Presentation and Leadership skills, Teamwork, Teaching skills

English (fluent, TOEFL 2011 (102/120)), Russian (basic), German (basic), Serbian (native)

\section{Extracurricular and NGOs Activities}

- Young Researchers of Serbia (Member)

- Karate Association of Serbia (Referee) / Karate Club "Ronin" (Coach, 1st dan)

- Faculty's Martial Art team (Captain)

- Rugby player at Universities of Belgrade, Lübeck, Göttingen and Vienna

- Taekwondo Club, Hochschulsport Göttingen (Member)

\section{Personal Interests}

Practical electronics, Mechanics, Photography, Traveling, Mountaineering, Camping, Fishing 
Translating Advanced Myocontrol for Upper Limb Prostheses from the Laboratory to Clinics 